Aus der Klinik für Nephrologie und Rheumatologie

(Prof. Dr. med. G.A. Müller)

der Medizinischen Fakultät der Universität Göttingen

\title{
Behandlungserfolg und Prognose des akuten Nierenversagens bei Patienten mit Sepsis und onkologischen Erkrankungen
}

\author{
INAUGURAL - DISSERTATION \\ zur Erlangung des Doktorgrades \\ der Medizinischen Fakultät der \\ Georg-August-Universität zu Göttingen
}

vorgelegt von

Alexander Mertens

aus

Hannover

Göttingen 2016 
Dekan:

Referent/in:

Ko-Referent/in:

Drittreferent/in:
Prof. Dr. rer. nat. H.K. Kroemer

Prof. Dr. med. D. Patschan

PD Dr. med. D. Heise

Prof. Dr. rer. nat. R. Hilgers

Datum der mündlichen Prüfung: 01.06.2017 


\section{Erstpublikation der Ergebnisse}

Teilergebnisse dieser Arbeit wurden bereits auszugsweise in einer Originalpublikation im Jahr 2013 veröffentlicht. Der Volltext dieser Publikation ist im Anhang zu dieser Arbeit eingefügt.

Heeg M, Mertens A, Ellenberger D, Müller GH, Patschan D (2013): Prognosis of AKI in malignant diseases with and without sepsis. BMC Anesthesiology 13, 76-81

\section{Abstract \\ Background:}

AKI significantly worsens prognosis of hospitalized patients. This is particularly the case in patients with sepsis. The risk for aquiring sepsis is significantly increased in malignant diseases. Aim of the present retrospective study was to analyze outcomes of tumor patients with sepsis and AKI.

\section{Methods:}

One-thousand and seventeen patients, treated at the ICU of the Department of Nephrology and Rheumatology of the University Hospital Göttingen from 2009 to 2011 were retrospectively analyzed for mortality, sepsis, AKI, need for renal replacement therapy (dialysis) and malignancies.

\section{Results:}

AKI occurred significantly more frequent in septic than in non-septic patients and in tumor as oposed to non-tumor patients. Mortaliy rates were higher in the respective latter groups. Mortality increased even further if patients suffered from a malignant disease with sepsis and AKI. Mortality rates peaked if dialysis treatment became mandatory. In non-solid tumors $100 \%$ of the patients died if they suffered drom sepsis and AKI. This was not the case in solid malignancies (mortality rate $56 \%$ ).

\section{Conclusions:}

We conclude that prognosis of tumor patients with AKI and sepsis is very poor. Mortality increases to almost $70 \%$ if diaylsis therapy is initiated. Non-solid tumors are associated with a $100 \%$ mortality if sepsis and AKI conincide.

Keywords: AKI; Sepsis; Malignancies; Mortality 


\section{Inhaltsverzeichnis}

Verzeichnis der Abbildungen

$\begin{array}{ll}\text { Verzeichnis der Tabellen } & \mathbf{v}\end{array}$

Abkürzungen vi

1 Einleitung 1

1.1 Historischer Rückblick . . . . . . . . . . . . . . . . . . . . 1

1.2 Die Bedeutung des akuten Nierenversagens in der intensivmedizinischen

Behandlung . . . . . . . . . . . . . . . . 2

1.3 Definition und Klassifizierung der Stadien des akuten Nierenversagens . . 4

1.4 Akutes Nierenversagen und Sepsis . . . . . . . . . . . . . . . . . . 5

1.5 Akutes Nierenversagen und maligne Neoplasien . . . . . . . . . . . . . . . 6

1.6 Fragestellungen und Ziel dieser Untersuchung . . . . . . . . . . . . . . . 6

2 Material und Methoden $\quad 8$

2.1 Patientenkollektiv und Rahmenbedingungen . . . . . . . . . . . . . 8

2.2 Ethikvotum und Datenschutz . . . . . . . . . . . . . . . . . . 9

2.3 Datenerfassung . . . . . . . . . . . . . . . . . . . 9

2.4 Diagnosen und Vorerkrankungen . . . . . . . . . . . . . . . . . . . . . . . 9

2.5 Selektive Krankheitsbilder und Diagnosen . . . . . . . . . . . . . . . 10

2.5.1 Akutes Nierenversagen (AKI; Acute Kidney Injury) . . . . . . . . 10

2.5.2 Sepsis - Begriffsbestimmungen und diagnostische Kriterien . . . . . 12

2.5.3 Maligne Tumorerkrankungen . . . . . . . . . . . . . . . . . . 14

2.6 Medikation . . . . . . . . . . . . . . . . . . . . . . . . 14

2.7 Laborbefunde . . . . . . . . . . . . . . . . . . . . . . . . . . . . . . . . . . . . . . .

2.8 Schweregrad von Erkrankungen (SAPS II) . . . . . . . . . . . . . . 16

2.9 Statistische Auswertung . . . . . . . . . . . . . . . . . . . . . 17

3 Ergebnisse $\quad \mathbf{2 0}$

3.1 Patientenkollektiv - Allgemeine Merkmale . . . . . . . . . . . . . . . 20

3.2 Erkrankungen und Diagnosen . . . . . . . . . . . . . . . . . . . . . . . . . . 23

3.3 Mortalität und Todesursachen . . . . . . . . . . . . . . . . . . 24

3.4 SAPS II - Klassifizierung des Schweregrades von Erkrankungen . . . . . . 27

3.5 Faktoren des Behandlungserfolgs . . . . . . . . . . . . . . . . 30

3.6 Akutes Nierenversagen . . . . . . . . . . . . . . . . 32 
3.7 Akutes Nierenversagen und Sepsis . . . . . . . . . . . . . . 36

3.8 Malignom . . . . . . . . . . . . . . . . . . . . 41

3.9 Akutes Nierenversagen und maligne Neoplasien . . . . . . . . . . . . . . 44

3.10 Akutes Nierenversagen bei Malignom und Sepsis . . . . . . . . . . . . . 47

3.11 Nierenersatztherapie bei Malignom und Sepsis . . . . . . . . . . . . . . . . . . 48

3.12 Akutes Nierenversagen bei Leberzirrhose . . . . . . . . . . . . . . 50

3.13 Zusammenfassung der wichtigsten Ergebnisse . . . . . . . . . . . 52

4 Diskussion $\quad \mathbf{5 3}$

4.1 Diagnose des AKI . . . . . . . . . . . . . . . 55

4.2 Prävalenz und Mortalität des AKI . . . . . . . . . . . . . . 56

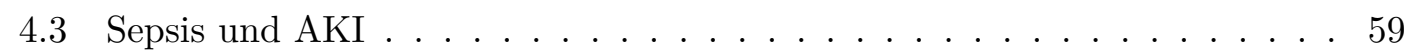

4.4 Maligne Neoplasien und AKI . . . . . . . . . . . . . . . . . . . . 60

4.5 Maligne Neoplasien, Sepsis und AKI . . . . . . . . . . . . . . . . . . 61

4.6 Nierenersatztherapie bei Malignom und Sepsis . . . . . . . . . . . . . . . 62

4.7 Leberzirrhose und AKI . . . . . . . . . . . . . . . . . . 63

$\begin{array}{lll}5 & \text { Zusammenfassung und Perspektiven } & 64\end{array}$

6 Literaturverzeichnis $\quad 66$

$\begin{array}{lr}\text { Anhang } & 76\end{array}$ 


\section{Abbildungsverzeichnis}

3.1 Behandlungsdauer - ICU . . . . . . . . . . . . . . . . . . 22

3.2 Patientenstruktur - Hauptdiagnosen und Begleiterkrankungen . . . . . . . 24

3.3 Prävalenz und Mortalität der Haupterkrankungen im Patientenkollektiv . 25

3.4 Mortalität im Patientenkollektiv . . . . . . . . . . . . . . . 26

3.5 Todesursachen im Patientenkollektiv . . . . . . . . . . . . . . . . . . . 27

3.6 Schweregrad von Erkrankungen - SAPS II . . . . . . . . . . . . . . . 28

3.7 SAPS II - Empirische kumulative Dichtefunktion . . . . . . . . . . . . . . 29

3.8 SAPS II - Histogramm und Verteilungsfunktion . . . . . . . . . . . . . . . . 30

3.9 Prävalenz und Mortalität des AKI . . . . . . . . . . . . . . . . . . . . . . . . . . . . . 33

3.10 Mortalität des AKI . . . . . . . . . . . . . . . . . . . . 34

3.11 Kreatinin-Konzentration bei Aufnahme und Entlassung . . . . . . . . 37

3.12 Prävalenz von AKI und Sepsis . . . . . . . . . . . . . . . . . . . . . 38

3.13 Verteilung bakterieller Erreger bei Sepsis . . . . . . . . . . . . . . . . . 39

3.14 Prävalenz und Mortalität von Sepsis und AKI . . . . . . . . . . . . . . . . . . . 41

3.15 Prävalenz der AKIN-Stadien und Sepsis . . . . . . . . . . . . . . . 42

3.16 Prävalenz und Mortalität maligner Neoplasien . . . . . . . . . . . . . . . . 44

3.17 Verteilung maligner Neoplasien . . . . . . . . . . . . . . . . . . . . . . . . . . . . . 45

3.18 Verteilung Hämoblastome . . . . . . . . . . . . . . . . . . . . 46

3.19 Prävalenz und Mortalität bei malignen Neoplasien und AKI . . . . . . . . 47

3.20 Prävalenz und Mortalität bei malignen Neoplasien, Sepsis und AKI . . . . 48

3.21 Nierenersatztherapie bei malignen Neoplasien und Sepsis . . . . . . . . . . 49

3.22 Nierenersatztherapie bei Hämoblastosen und Sepsis . . . . . . . . . . . . 50 


\section{Tabellenverzeichnis}

2.1 Klassifizierung nach Organsystemen _ . . . . . . . . . . . . . . 10

2.2 Klassifikations-System des AKI . . . . . . . . . . . . . . . . . . . . . 11

2.3 Nierenersatztherapie - Indikation . . . . . . . . . . . . . . . . . . . . . . . 11

2.4 SIRS - Diagnostische Kriterien . . . . . . . . . . . . . . . . . . . 12

2.5 Organversagen - Diagnostische Kriterien . . . . . . . . . . . . . . 13

2.6 Laborwerte und Referenzintervalle . . . . . . . . . . . . . . . . . . 15

2.7 Bemessungsgrundlagen - SAPS II . . . . . . . . . . . . . . . . . . . . 18

2.8 Bemessungsgrundlagen - SAPS II Fortsetzung . . . . . . . . . . . . . . 19

3.1 Patientenstruktur - Übersicht . . . . . . . . . . . . . . . . . . . 21

3.2 Behandlungserfolg - AKI . . . . . . . . . . . . . . . . 31

3.3 Patientencharakteristika - AKI . . . . . . . . . . . . 35

3.4 Laborwerte - AKI . . . . . . . . . . . . . . . . . . 37

3.5 Patientencharakteristika - AKI und Sepsis . . . . . . . . . . . . . 40

3.6 Patientencharakteristika - Maligne Neoplasien . . . . . . . . . . . . . . . . 43

3.7 Patientencharakteristika - Solide und hämoblastische Neoplasien . . . . . 45

3.8 Patientencharakteristika - Leberzirrhose . . . . . . . . . . . . . . 51 


\section{Abkürzungen}

$\begin{array}{ll}\text { AKI } & \text { Akutes Nierenversagen (acute kidney injury) } \\ \text { AKIN I-III } & \text { Klassifikation der Stadien des akuten Nierenversagens } \\ \text { ICU } & \text { Intensivstation (intensive care unit) } \\ \text { SIRS } & \text { Systemisch-inflammatorisches Response-Syndrom } \\ & \text { (systemic inflammatory response syndrome) } \\ \text { SAPS II } & \text { Simplified Acute Physiology Score } \\ \text { UMG } & \text { Universitätsmedizin Göttingen } \\ R & \text { Quotenverhältnis (odds ratio) } \\ {\left[K r_{s}\right]_{1}} & \text { Kreatinin-Konzentration im Serum } \\ \Delta\left[K r_{s}\right]_{r e l} & \text { Relative Änderung der }\left[K r_{s}\right] \\ \Delta\left[K r_{s}\right]_{a b s} & \text { Absolute Änderung der }\left[K r_{s}\right] \\ \dot{U} & \text { Urinmenge pro Zeiteinheit } \\ m, w & \text { Geschlecht: männlich, weiblich } \\ h & \text { Stunde(n) } \\ d & \text { Tag(e) } \\ y & \text { Jahr(e) }\end{array}$




\section{Kapitel 1}

\section{Einleitung}

\subsection{Historischer Rückblick}

Das Versagen der Nierenfunktion ist den Ärzten im 17. und 18. Jahrhundert schon unter dem Krankheitsbegriff ischuria renalis bekannt gewesen, zu einem Zeitpunkt, als die anatomisch-physiologischen Grundlagen der Funktion der Niere noch nicht näher erforscht waren. Unterschiedliche Verlaufsformen, die ursächlich nach heutiger Lesart in prärenal, intrarenal und postrenal eingeteilt werden, waren den Ärzten bereits in dieser Zeit durchaus geläufig, ebenso wie eine entsprechende galenische Arzneimitteltherapie. So finden sich in den medizinischen Lehrbüchern aus der 1. Hälfte des 19. Jahrhunderts unter dem Oberbegriff ischuria renalis zahlreiche Unterbegriffe für die verschiedenartigen Verlaufsformen (Graefe 1839; Eberle 1841). Ein wesentlicher Beitrag zum Verständnis der Nierenfunktion wurde durch mikroskopische Untersuchungen von Jakob Henle (1809 - 1885), dem Begründer der Histologie, erbracht, der von 1852 bis zu seinem Tod Direktor des Instituts für Anatomie der Georg-August-Universität in Göttingen war.

Die akute Verlaufsform der ischuria renalis, das akute Nierenversagen mit Eintritt des Todes innerhalb von wenigen Tagen, mit anschaulicher Beschreibung der klinischen Symptome, des zeitlichen Verlaufs und der Therapie findet sich in den Veröffentlichungen englischer Ärzte aus den Jahren 1821 und 1840 (Abercombie 1821; Fife 1840). Als Komplikation einer Malaria falciparum-Epidemie wurde das akute Nierenversagen bereits 1911 beschrieben (Yorkes und Nauss 1911). Unter dem Begriff acute Bright's disease wurden einige Fälle von akutem Nierenversagen in William Oslers Standardwerk The Principles and Practice of Medicine bereits 1912 beschrieben (Osler 1912). Während des 1 . Weltkriegs wurde das akute Nierenversagen bei schweren traumatischen Verwundungen auch als war nephritis bezeichnet (Davies und Weldon 1917), geriet danach als eigenstängige Erkrankung zunächst aber wieder in Vergessenheit. Unter dem Eindruck 
der schweren Kompressions-Verletzungen (crush syndrome) bei Patienten anlässlich der verheerenden Bombenangriffe auf London im Jahre 1941 (Bywaters und Beall 1941) geriet das akute Nierenversagen wieder in den Blickpunkt der wissenschaftlichen Forschung und wurde unter dem Begriff acute renal failure im Jahr 1951 als klinische Entität im Rahmen schwerer traumatischer Verletzungen eingeführt (Smith 1951).

In der Folgezeit haben sich unterschiedliche diagnostische Kriterien entwickelt, so dass in den wissenschaftlichen Veröffentlichungen die Angaben zur Inzidenz, Prognose und Mortalität uneinheitlich waren und einen weiten Bereich umfassten. Obwohl das akute Nierenversagen (acute kidney injury, AKI) - die innerhalb eines kurzen Zeitraums akut verminderte oder völlig sistierende Nierenfunktion - eine klinische Entität darstellt, deren Diagnose auf der Basis einfacher Kenngrößen an Hand von Laborwerten (Blut, Urin) erfolgt, haben sich in der Vergangenheit zur Definition des AKI unterschiedliche diagnostische Kriterien etabliert, die sich vorwiegend am zeitlichen Anstieg der SerumKreatinin-Konzentration $\left(\left[\mathrm{Kr}_{s}\right]\right)$ und ausgeschiedener Urinmenge $(\dot{U})$ orientierten. Zeitweilig waren in der Literatur mehr als 35 verschiedene Definitionen und Klassifikationen angegeben (Kellum et al. 2002). Entsprechend den unterschiedlichen Klassifikationen zur Inklusion von Patienten mit AKI waren die Angaben zur Inzidenz und Mortalität uneinheitlich (Singbartl und Kellum 2012). Auf einer ersten internationalen KonsensusKonferenz wurden 2004 die bis dahin bestehenden 30 unterschiedlichen Definitionen des akuten Nierenversagens durch eine einheitliche systematische Definition und Stadieneinteilung ersetzt (RIFLE-Kriterien, Risk - Injury - Failure - Loss - ESRD (End Stage Renal Disease); Bellomo et al. 2004). Auf einer weiteren Konsensus-Konferenz des Acute Kidney Injury Network (AKIN) im Jahre 2007 wurden geringfügig abweichende neue diagnostische Kriterien und Kennmerkmale für den Schweregrad der Erkrankung unter dem Begriff (acute kidney injury, AKI) definiert (Mehta et al. 2007; Ratanarat et al. 2013). Mit den AKIN-Kriterien werden auch leichtere Formen des AKI erfaßt. Hierbei war die Absicht führend, durch die Empfehlung einer vereinheitlichten Klassifizierung eine bessere Vergleichbarkeit der wissenschaftlichen Ergebnisse im internationalen Rahmen zu ermöglichen. Vor diesem Hintergrund wurde erstmals im Jahre 2012 eine Leitlinie zur Diagnostik und Therapie des AKI durch die KDIGO (Kidney Disease: Improving Global Outcomes) veröffentlicht (KDIGO 2012).

\subsection{Die Bedeutung des akuten Nierenversagens in der in- tensivmedizinischen Behandlung}

Das AKI stellt, ungeachtet des Umstands, dass die Diagnose vermeintlich einfach gestellt werden kann, auch in heutiger Zeit ein schwerwiegendes Erkrankungsbild und 
therapeutisches Problem in der Intensivmedizin dar. Dies betrifft besonders die frühzeitige Diagnose der Erkrankung und die Effizienz der therapeutischen Behandlungskonzepte. Generell betrachtet, sind die Behandlungserfolge (outcome) über den Zeitraum zurückliegender Jahre nahezu unverändert und dementsprechend die Prognose des AKI insgesamt ungünstig geblieben.

Es mag durchaus erstaunlich erscheinen, dass - ungeachtet zahlreicher Veröffentlichungen und Übersichtsdarstellungen - verläßliche Angaben zur Epidemiologie des AKI, d.h. zu Inzidenz und Mortalität, nicht zur Verfügung stehen. Dies ist auf mannigfaltige Gründe zurückzuführen, die im Wesentlichen auf Unterschiede in den diagnostischen Kriterien und in der unsystematischen Auswahl der Patientenkollektive zurückzuführen sind. Die Inzidenz des AKI beträgt bei hospitalisierten Patienten 5-8\% (Hou et al. 1983; Shusterman et al. 1987; Brivet et al. 1996; Liano und Pascual 1996; Liano et al. 1998; Guerin et al. 2000; Nash et al. 2002; Metha et al. 2004; Uchino et al. 2005b), bei Patienten im intensivmedizinischen Bereich 5-25\% und liegt bei selektierten Patientenkollektiven, z.B. bei Patienten nach kardiochirurgischen Eingriffen, bei 40-60\% (Harris et al. 2015). Das zunehmende Lebensalter der Patienten, auch verbunden mit vermehrter Multimorbidität, sowie ein intensiveres Verständnis von Pathophysiologie und Risikofaktoren (Sepsis, Röntgen-Kontrastmittel, nephrotoxische Antibiotika, Herz- und Aneurysma-Chirurgie) und ihrer prognostischen Bedeutung für den Verlauf des AKI haben zu einer weiter ansteigenden Inzidenz des AKI geführt (Waikar et al. 2008; Rewa und Bagshaw 2014; Siew und Davenport 2015). Bei retrospektiver Betrachtung von Inzidenz und Mortalität des AKI ist nicht eindeutig zu klären, ob es sich um eine tatsächliche Zunahme oder um eine scheinbare Zunahme handelt, da verschiedene Faktoren, z.B. verbesserte Diagnostik, demografische Altersentwicklung der Patientenkollektive mit Prävalenz von Herz- und Tumorerkrankungen sowie verbesserte therapeutische Optionen eine schlüssige Beurteilung erschweren. Ungeachtet großer Fortschritte in der intensivmedizinischen Therapie ist das AKI immer noch eine häufige und in der Therapie aufwendige Nierenerkrankung und weiterhin ein wesentlicher Faktor für die Morbidität und Letalität. Die Mortalitätsrate liegt bei 30-80\% und ist trotz intensiver Bemühungen in den letzten Jahrzehnten unverändert geblieben (Hou et al. 1983; Brivet et al. 1996; Liano und Pascual 1996; Liano et al. 1998; Guerin et al. 2000; Uchino et al. 2005b; Ympa et al. 2005; Xue et al. 2006; Waikar et al. 2006). Der Einsatz von verschiedenen Biomarkern zur Früherkennung von Tubulusschädigungen oder tubulärer Dysfunktion bzw. die Analyse tubulärer Enzyme im Rahmen der Entwickung eines AKI haben bisher keine Bedeutung als geeignete Frühindikatoren erfahren. Nach wie vor werden die dynamischen Veränderungen von Serum-Kreatinin-Konzentration $\left(\left[\mathrm{Kr}_{s}\right]\right)$ und ausgeschiedener Urinmenge $(\dot{U})$ als diagnosische Kriterien herangezogen, unabhängig von der Tatsache, dass $\left[\mathrm{Kr}_{s}\right]$ ein Index der glomerulären Filtration und kein Indikator für eine Nierenzell-Schädigung 
per se darstellt. Neue spezifische Behandlungsstrategien, pharmakologische Ansätze oder nierenprotektive Verfahren sind bisher nicht entwickelt worden. In diesem Zusammenhang ist auch zu erwähnen, dass repräsentative Tiermodelle für das AKI nicht entwickelt werden konnten (Kellum 2011; Marugan und Kellum 2011).

\subsection{Definition und Klassifizierung der Stadien des akuten Nierenversagens}

Das AKI bezeichnet die innerhalb eines kurzen Zeitraums (Stunden, Tage) verminderte oder völlig eingeschränkte Nierenfunktionsleistung, die bei effektiver therapeutischer Überbrückung durch Dialyseverfahren potentiell reversibel ist, obwohl eine vollständige restitutio ad integrum nicht immer zu erreichen ist. Bei einer Einschränkung der Nierenfunktion wird die paarweise Anordnung der Nieren nicht berücksichtigt. Im Allgemeinen wird davon ausgegangen, dass die Ursache einer gestörten Nierenfunktion beide Nieren gleichermaßen betrifft (obwohl dies praktisch nicht immer der Fall sein muss). In den meisten Fällen handelt es sich beim AKI nicht um eine Primär-Erkrankung sui generis, vielmehr stellt das AKI häufig eine zusätzlich aufretende Sekundär-Komplikation einer vorbestehenden Grunderkrankung dar. Das AKI führt in der Regel - in Abhängigkeit von der Therapie und Effektivität einer funktionellen Nierenersatztherapie (Dialyseverfahren) - zu einer erhöhten zusätzlichen Komplexität der Grunderkrankung mit der Notwendigkeit des Einsatzes ergänzender therapeutischer Maßnahmen. Ätiologisch wird das AKI in prärenale (Volumenmangel, Hypotonie, Herzinsuffizienz), intrarenale (vasomotorisch, renoparenchymatös, tubulotoxisch, vaskulär) und postrenale (Obstruktion der ableitenden Harnwege) Ursachen eingeteilt.

Beispielhaft mag als Primärerkrankung die chronische Niereninsuffizienz gelten, wobei ungeachtet unterschiedlicher Ätiologie - bei progressivem Verlauf oder Dekompensation dieser Erkrankung der Eintritt eines AKI als Langzeitkomplikation immer eine schwerwiegende Komplikation darstellt. So verursachen bereits geringe Einschränkungen der Nierenfunktion eine erhöhte Komplikationsrate mit potentieller Entwicklung weiterer negativer Folgeerkrankungen und konsekutiv erhöhter Mortalität. Das AKI ist aber nicht allein auf vorbestehende Nierenerkrankungen beschränkt, sondern kann als belastendes Risiko im Rahmen zahlreicher andersartiger Grunderkrankungen und Umstände auftreten (Ischämie, Sepsis, Herzerkrankungen, Lebererkrankungen, Herz- und Gefäßchirurgie, Pneumonie). Das AKI ist demgemäß das Ergebnis von intrinsischer Suszeptibilität und Risikofaktoren eines Patienten sowie dessen extrinsischer Exposition gegenüber kausalen, auslösenden Faktoren. 
Die hohe Mortalität ist aber nicht allein durch das AKI per se zu erklären, vielmehr ist das AKI mit dem Auftreten von tertiären nicht-renalen Komorbiditäten assoziiert. Hierzu gehören verschiedene Zustände von Kreislauf- und Herzinsuffizienz und eine interkurrierende Sepsis, Erkrankungen die ihrerseits zum Tod führen können (Levy et al. 1996). Auch konnten in den zurückliegenden Jahren verschiedene Risikofaktoren identifiziert werden (kardiogener Schock, kardiovaskuläre chirurgische Eingriffe, Nephrotoxine), deren Assoziation mit der Entwicklung eines AKI nachweisbar war (Uchino et al. 2005a,b). Trotz zunehmender Hinweise auf Risikofaktoren für die Entwicklung eines AKI besteht nach wie vor ein unzureichendes Verständnis der Pathophysiologie unter den vorbezeichneten Umständen und gilt insbesondere für das Sepsis-assoziierte AKI sowie das AKI im Rahmen maligner neoplastischer Erkrankungen (Singbartl und Kellum 2012). Der Behandlungserfolg des AKI ist daher in entscheidendem Maße von der Art der zugrunde liegenden Grunderkrankung, ihrer Schwere und ihrer Dauer abhängig. Es mag auch gerechtfertigt erscheinen, ein AKI nicht allein als assoziierte Komplikation bei einer bestehenden Grundkrankheit zu betrachten, sondern insbesondere auch im Hinblick auf zahlreiche Folgeerkrankungen und Spätkomplikationen als eigenständiges Syndrom zu verstehen. In diesem Sinne ist es nicht nur eine semantische Frage, ob bei der Mortalität des AKI ein Patient "am" AKI oder "mit" einem AKI verstorben ist.

\subsection{Akutes Nierenversagen und Sepsis}

Die Sepsis ist eine schwerwiegende Erkrankung, gekennzeichnet durch eine systemische Inflammation, ausgelöst von pathogenen Keimen (Bakterien, Bakterientoxinen oder Pilzen), welche aus einem Infektionsherd in die Blutbahn gelangen. Es kommt zur Aktivierung von Kaskadensystemen und speziellen Zellsystemen mit Freisetzung humoraler und zellulärer Mechanismen. Eine Sepsis kann zu Komplikationen mit topographisch regionalen Organ-Funktionstörungen führen oder in generalisierter Form ein Multiorganversagen auslösen. Nach den Konsensuskriterien der internationalen Sepsis-Konferenzen aus den Jahren 1991 und 2001 (Bone et al. 1992; Levy et al. 2003) werden unter dem Oberbegriff Sepsis verschiedene Schweregrade der Erkrankung verstanden: (i) Infektion, (ii) Systemisch-inflammatorisches Response-Syndrom (SIRS), (iii) Sepsis, (iv) Schwere Sepsis, (v) Septischer Schock. Die Klassifizierung nach verschiedenen Stufen einer septischen Erkrankung bedeutet aber lediglich, dass es sich hierbei um verschiedene Ausprägungen einer kontinuierlichen Krankheitsentwicklung handelt.

Von besonderer Bedeutung ist das akute septische Nierenversagen, das in seiner Pathogenese vom nicht-septischen AKI abweicht (Wan et al. 2008). Die Mortalität des 
Sepsis-assoziierten AKI liegt nach den Ergebnissen verschiedener Untersuchungen in einem weiten Bereich $(28-90 \%)$ und stellt daher in der intensivmedizinischen Therapie ein besonderes Problem und eine besondere Herausforderung dar (Bagshaw et al. 2007; Waikar et al. 2008; Bougle und Duranteau 2011; Cartin-Ceba et al. 2012; Zarbock et al. 2014). Die Prognose des septischen AKI wird durch eine Dialysetherapie nicht entscheidend verbessert (Ricci und Ronco 2009).

\subsection{Akutes Nierenversagen und maligne Neoplasien}

Das Risiko einer Sepsis wird maßgeblich von der Immunkompetenz bzw. der Immundefizienz des Patienten bestimmt. Eine Einschränkung der Immunkompetenz liegt typischerweise bei Patienten mit malignen Tumorerkrankungen vor. Das Infektionsrisiko ist als potentielle Komplikation bei Tumorpatienten unter der immunsupressiven Therapie mit Chemotherapeutika drastisch erhöht und kann einen begünstigenden Faktor für die Entwicklung eines AKI darstellen. Ebenso kann die potentielle Nephrotoxität der Chemotherapeutika einen direkten Einfluss auf die Entwicklung eines AKI haben. Neuere klinische Untersuchungen haben Hinweise auf eine höhere Inzidenz des AKI bei malignen Neoplasien erbracht, insbesondere bei hämoblastischen Neoplasien (Bagshaw et al. 2009; Christiansen et al. 2011). Andere Untersuchungen konnten dagegen weder für maligne solide Neoplasien noch für hämoblastische Neoplasien als unabhängige Risikofaktoren des AKI eine erhöhte Mortalität feststellen (Hoste et al. 2006; Maccariello et al. 2011; Salahudeen et al. 2013). Die Frage nach Prognose und Mortalität des AKI in Bezug auf maligne Neoplasien (mit oder ohne die erweiterte Komplexität einer gleichzeitigen Sepsis) ist bisher nur unzureichend beantwortet, ebensowenig wie die Frage der Sinnhafigkeit des Einsatzes von Nierenersatzverfahren bei prognostisch infausten Krankheiszuständen.

\subsection{Fragestellungen und Ziel dieser Untersuchung}

In dieser Untersuchung sollen die Behandlungsergebnisse des akuten Nierenversagens unter besonderer Berücksichtigung schwerer Grunderkrankungen, namentlich der Sepsis und maligner Tumorerkrankungen, untersucht werden. Bei den malignen Neoplasien werden solide Tumoren und Hämoblastosen gesondert betrachtet. Die Grundlage hierfür ist die retrospektive Analyse der Patienten-Dokumentationen der nephrologischen Intensivstation der UMG über einen 3-Jahres-Zeitraum von 2009 - 2011. Es sollen die Fragen der Prävalenz und der Mortalität des AKI als Sekundärkomplikation bzw. Komorbidität bei bestehenden schweren Grundkrankheiten sowie die Frage von Risikofaktoren dieser 
und anderer Erkrankungen und Zustände behandelt werden. Ein Schwerpunkt der Analyse liegt dabei auf der Untersuchung der Komorbidität des AKI bei Sepsis und maligen Neoplasien. In diesem Zusammenhang soll auch die Frage des Einsatzes therapeutischer Nierenersatzverfahren beleuchtet werden. 


\section{Kapitel 2}

\section{Material und Methoden}

\subsection{Patientenkollektiv und Rahmenbedingungen}

Die vorliegende Arbeit ist eine retrospektive Untersuchung der Behandlungserbebnisse der Klinik für Nephrologie und Rheumatologie der Universitätsmedizin Göttingen (UMG). Sie umfaßt alle Patienten, die über einen 3-Jahres-Zeitraum vom 01.01.2009 bis zum 31.12.2011 auf der nephrologischen Intensivstation behandelt wurden $(N=1017)$. Hieraus ergibt sich, dass im Mittel $\sim 340$ Patienten/Jahr mit einer durchschnittlichen Liegedauer von $\sim 8$ Tagen behandelt wurden und auf der 8-Bettenstation $\sim 1$ Patient/Tag neu aufgenommen wurde. Von der Analyse mussten 20 Patienten ausgeschlossen werden, deren Dokumentation unvollständig war. Alle erfaßten Daten wurden aus der Datenbank der Klinik für Nephrologie und Rheumatologie der UMG entnommen. Die Grundlage der Datenerhebung war die Auswertung der klinikinternen ArztbriefDatenbank, der elektronischen Patienten-Akten sowie der elektronischen Befund-Datenbank. Laborbefunde und Befunde anderer Untersuchungsverfahren wurden dem in enger Kooperation mit der UMG entwickelten Dokumentationsprogramm ixserv (ixmid Software Technologie $\mathrm{GmbH}$ ) entnommen. Der Betrachtungszeitraum der Untersuchungen betrifft ausschließlich den Zeitraum der Behandlung auf der Intensivstation. Weiterführende Beobachtungen nach der Entlassung bzw. Verlegung von der Intensivstation waren nicht möglich, da die Patientendokumentation insbesondere bei Verlegung der Patienten in auswärtige Krankenhäuser nicht zur Verfügung stand. 


\subsection{Ethikvotum und Datenschutz}

Der Antrag (DOK-62-2012 vom 05.11.2012) zur Überprüfung des Forschungsvorhabens wurde durch die Ethik-Kommission der UMG dahingehend beantwortet, dass ein Forschungsvorhaben wie dieses, worin ausschließlich bereits existierende Behandlungsunterlagen der UMG wissenschaftlich ausgewertet werden, keiner Befassung und ggf. Zustimmung der Ethik-Kommission bedarf. Gemäß den allgemeinen Geschäftsbedingungen der UMG, ist die wissenschaftliche Auswertung des Datenmaterials ohne Zustimmung des jeweiligen Patienten statthaft, sofern die Daten in anonymisierter Form erhoben werden.

Eine beratende Belehrung des Untersuchers und Verfassers dieser Arbeit bei dem zuständigen Datenschutzbeauftragten der UMG ist am 06.11.2012 erfolgt. Alle Patientendaten, die im Rahmen dieser Arbeit erhoben wurden, wurden in streng anonymisierter Form erfasst.

\section{$2.3 \quad$ Datenerfassung}

Die Patientendaten wurden aus den internen medizinischen Dokumentationssystemen der UMG entnommen und in das Tabellenkalkulationsprogramm Excel (Version 2007) überführt. Patientendaten, die in anderen Dokumentationssystemen gespeichert wurden, wurden mit Unterstützung der Abteilung für Informationstechnologie und Medizinisches Controlling der UMG erfasst und in die Arbeitsblätter eingefügt. Berechnungen und Abbildungen wurden mit Matlab (Version 2011, The MathWorks, Inc.) ausgeführt. Die statistische Auswertung der Daten erfolgte mit dem Statistikprogramm Statistica (Version 12, StatSoft Inc.). Das Manuskript dieser Arbeit wurde mit dem LaTeX-Textsatzsystem (MiKTeX Version 2.9) geschrieben.

\subsection{Diagnosen und Vorerkrankungen}

Für jeden Patienten wurde erfasst, ob die stationäre Aufnahme aus medizinischer oder chirurgischer Indikation erfolgte. Weiterhin wurden die Hauptdiagnose(n) und sämtliche Begleiterkrankungen aufgezeichnet. Hierzu wurde zunächst eine auf Körperorgane bezogene Klassifizierung der Erkrankungen nach den Diagnosen der Haupterkrankungen vorgenommen.

Jeder Patient konnte 1, 2 oder mehrere Erkrankungen aus den klassifizierten Gruppen haben. Alle Nebendiagnosen, die aus Vorbefunden, Befunden während des stationären 
- kardiovaskulär

- pneumonologisch

- nephrologisch

- onkologisch

- hepatisch

- infektiös

- andere

TABELlE 2.1: Klassifizierung nach Organsystemen.

Aufenthalts oder aus dem Entlassungsbrief hervorgingen, wurden gesondert dokumentiert.

\subsection{Selektive Krankheitsbilder und Diagnosen}

\subsubsection{Akutes Nierenversagen (AKI; Acute Kidney Injury)}

Das Vorliegen eines akuten Nierenversagens (AKI), d.h. die funktionelle, potentiell reversible Einschränkung der Nierenfunktion wurde den Aufzeichnungen der Laborwerte harnpflichtiger Substanzen im Blut $\left(\left[K r_{s}\right]\right)$ und der Urinmenge $(\dot{U})$ sowie deren zeitlicher Entwicklung entnommen. Grundsätzlich ist das AKI eine funktionell diagnostische Entität und nicht an Zustände mit vorbestehenden schweren Nierenerkrankungen und Niereninsuffizienz gebunden. Für die Diagnose wurden die Grenzwert-Kriterien herangezogen, die auf der Basis von Empfehlungen der Acute Kindney Injury Network Group (AKIN-Kriterien - Mehta et al. 2007; Ratanarat et al. 2013) international verbreitet und akzeptiert sind. Die Kriterien sind in Tab. 2.2 zusammengefaßt. Nach Ausmaß der Veränderungen der Laborwerte und ihrer zeitlichen Dynamik werden die Schweregrade I - III unterschieden.

Ein AKI mit Schweregrad I liegt vor: a) bei einem relativen Anstieg des $\left[K r_{s}\right]$ um den Faktor $1-2\left(\Delta\left[K r_{s}\right]_{r e l}\right)$ bezogen auf einen bekannten (evtl. bereits erhöhten) oder angenommenen Ausgangswert und einer zeitlichen Entwicklung von 7 Tagen; oder $b$ ) bei einem schnellen Anstieg der Absolutkonzentration $\left(\Delta\left[K r_{s}\right]_{a b s} \geq 0.3 \mathrm{mg} / \mathrm{dl}\right)$ innerhalb von 48 Stunden; oder $c$ ) bei grenzwertiger Unterschreitung der Urinausscheidung $(\dot{U}<0.5 \mathrm{ml} / \mathrm{kg} / \mathrm{h})$ über 6 Stunden. Zur Diagnose des AKI genügt die Erfüllung von einem der unter $a$ ) - c) aufgezählten Kriterien. Für die AKIN-Stadien II und III gelten analog die entsprechenden Parameter mit quantitativ höheren Werten für die zeitbezogene Veränderung der jeweiligen Parameter. 


\begin{tabular}{|c|c|c|}
\hline \multicolumn{3}{|c|}{ AKIN-Kriterien } \\
\hline Stadien & Serum-Kreatinin $\left[K r_{s}\right]$ & Urinausscheidung $(\dot{U})$ \\
\hline $\mathrm{I}$ & $\begin{array}{c}\Delta\left[K r_{s}\right]_{r e l}=1,5-2[/ 7 d] \\
\text { oder } \\
\Delta\left[K r_{s}\right]_{a b s} \geq 0,3 \mathrm{mg} / \mathrm{dl}[/ 48 \mathrm{~h}]\end{array}$ & $\dot{U}<0,5 \mathrm{ml} / \mathrm{kg} / \mathrm{h}[/ 6 \mathrm{~h}]$ \\
\hline II & $\Delta\left[K r_{s}\right]_{r e l}=2-3[/ 48 h]$ & $\dot{U}<0,5 \mathrm{ml} / \mathrm{kg} / \mathrm{h}[/ 12 \mathrm{~h}]$ \\
\hline III & $\begin{array}{c}\Delta\left[K r_{s}\right]_{a b s}>3[/ 7 d] \\
\text { oder } \\
{\left[K r_{s}\right]>4 \mathrm{mg} / \mathrm{dl}} \\
\text { und } \\
\Delta\left[K r_{s}\right]_{a b s} \geq 0,5 \mathrm{mg} / \mathrm{dl}[/ 48 \mathrm{~h}]\end{array}$ & $\begin{array}{l}\dot{U}<0,3 \mathrm{ml} / \mathrm{kg} / \mathrm{h}[/ 24 \mathrm{~h}] \\
\text { oder } \\
\dot{U}=0,0 \mathrm{ml} / \mathrm{kg} / \mathrm{h}[/ 12 \mathrm{~h}]\end{array}$ \\
\hline
\end{tabular}

TABELLE 2.2: Klassifikations-System des akuten Nierenversagens (Acute Kidney Injury Network), modifiziert nach Mehta et al. 2007.

Bei Vorliegen der AKI-Diagnose wurde eine Sub-Klassifizierung der Patienen nach vorbestehendem AKI bzw. eines sich während des Aufenthalts auf der ICU entwickelten AKI vorgenommen. Patienten mit terminaler Niereninsuffizienz wurden, ungeachtet der jeweiligen Genese, ebenfalls in die Studie einbezogen.

Unter der Bezeichnung Nierenersatzverfahren wurden alle extrakorporalen Dialyseverfahren sowie die Peritonealdialyse kollektiv zusammengefaßt. Die Indikation für eine Nierenersatztherapie war bei Vorliegen eines oder mehrerer Kriterien gegeben:

- Serum-Kreatinin Anstieg $\Delta\left[K r_{s}\right]_{a b s}>3,0 \mathrm{mg} / \mathrm{dl}$ und/oder

- Harnstoffanstieg > $100 \mathrm{mg} / \mathrm{dl}$ durch mangelnde Urinausscheidung. und/oder

- klinische Zeichen der Hypervolämie

TABELLE 2.3: Indikation der Nierenersatztherapie. 


\subsubsection{Sepsis - Begriffsbestimmungen und diagnostische Kriterien}

Die diagnostischen Kriterien für das Krankheitsbild der Sepsis wurden im Jahre 1992 anläßlich einer Internationalen Sepsis-Konferenz (American College of Chest Physicians / Society of Critical Care Medicine - ACCP/SCCM; Bone et al. 1992; Levy et al. 2003) definiert und gelten international als weithin verbindliche Empfehlung. Danach werden unter dem Oberbegriff Sepsis in differenzierter Form verschiedene Krankheitszustände unterschiedlicher Schweregrade verstanden.

\section{Infektion}

Unter einer Infektion werden reaktive entzündliche pathologische Prozesse des Organismus verstanden, die in a priori sterilem Gewebe durch kritische Ausbreitung mikrobiologischer Erreger in systemischer oder organbezogener Weise bedingt sind. Hierzu gehören auch Prozesse in ansonsten unsterilen Geweben, z.B. Darm, ebenso wie Prozesse, die indirekt durch Stoffwechselprodukte eines Erregers, z.B. Toxine, hervorgerufen werden.

\section{Systemisch-inflammatorisches Response-Syndrom - SIRS}

Als systemisch-inflammatorisches Response-Syndrom (systemic inflammatory response syndrome - SIRS) wird eine entzündliche Systemreaktion des Organismus bezeichnet, die als Folge allgemein schwerwiegender Ursachen unterschiedlicher Ätiologie ausgelöst wird. Zu den Krankheitsbildern gehören infektiöse Zustände, Schock, Verbrennungen, große Operationen oder schwere traumatische Schädigungen.

Die Diagnose eines SIRS wird gestellt, wenn $\geq 2$ der nachfolgenden Kriterien erfüllt sind:

- Hyperthermie $\mathrm{T}_{b}>38{ }^{\circ} \mathrm{C}$ oder Hypothermie $\mathrm{T}_{b}<36{ }^{\circ} \mathrm{C}$

- Herzfrequenz HR $>90 \mathrm{~min}^{-1}$

- Tachypnoe $\mathrm{BR} \geq 20 \mathrm{~min}^{-1}$ oder $\mathrm{CO}_{2}$-Partialdruck $\mathrm{PaCO}_{2} \leq 32 \mathrm{~mm} \mathrm{Hg}$

- Leukozytose $>12000 / \mu l$ oder Leukopenie $\leq 4000 / \mu l$ oder

Linksverschiebung im Diff.-Blutbild $>10 \%$ unreife neutrophile Granulozyten

TABELLE 2.4: Diagnostische Kriterien des SIRS.

\section{Sepsis}

Unter der Definition Sepsis wird maßgeblich das Vorliegen einer Infektion mit erfüllten SIRS-Kriterien verstanden:

$$
\text { Sepsis }=\text { SIRS infektiöser Genese }=\text { Infektion }+ \text { SIRS }
$$


Voraussetzung für die Diagnose einer Sepsis ist daher immer der Nachweis einer mikrobiologischen Infektion oder im negativen Fall durch klinische Kriterien. Der Nachweis einer Sepsis bzw. eines SIRS infektiöser Genese ist durch eine positive Blutkultur (aerobes und anaerobes Paar) zu führen. Bei positiver Blutkultur müssen zusätzlich $\geq 2$ SIRSKriterien (Tab. 2.4) erfüllt sein. Bei negativer Blutkultur müssen alle 4 SIRS-Kriterien erfüllt sein.

\section{Schwere Sepsis}

Unter dem Begriff schwere Sepsis wird die Kombination von Sepsis bzw. SIRS infektiöser Genese mit der zusätzlichen Kombination eines Organversagens verstanden:

$$
\text { schwere Sepsis }=\text { Sepsis }+ \text { Organversagen }
$$

Der Begriff Organversagen ist allerdings sehr breit gefaßt und ein unmittelbarer Organbezug nicht explizit gegeben. Für die Zustände einzelner oder mehrerer Organfunktionsausfälle und deren Kriterien gelten folgende Bedingungen:

- Akute Encephalopathie: eingeschränkte Vigilanz, Desorientierung, motorische Unruhe, Delirium

- Arterielle Hypotension, Schock:

$\mathrm{RR}_{\text {syst }}<90 \mathrm{mmHg}$ oder mittlerer art. Blutdruck (MAP) $\leq 70 \mathrm{~mm} \mathrm{Hg}$ über Zeitraum von $1 h$ bzw. von $2 h$ bei Einsatz von Vasopressoren und adäquater Volumen-Ersatztherapie

- Relative oder absolute Thrombozytopenie:

Thrombozyzenzahl $<100000 / \mu l$ oder Thrombozytenabfall $>30 \%$ in $24 h$

- Arterielle Hypoxämie:

$\mathrm{PaO}_{2} \leq 75 \mathrm{~mm} \mathrm{Hg}$ bei Raumluftatmung oder $\mathrm{PaO}_{2} / \mathrm{F}_{i} \mathrm{O}_{2}$-Verhältnis $\leq 250 \mathrm{~mm} \mathrm{Hg}$ bei $\mathrm{O}_{2}$-Applikation

- Renale Dysfunktion:

Kreatininanstieg $\Delta\left[K r_{s}\right]_{r e l} \geq 2$ oder Oligurie mit Diurese $\dot{U} \leq 0,5 \mathrm{ml} / \mathrm{kg} / \mathrm{h}$

- Metabolische Azidose:

Base Excess $\leq-5 \mathrm{mmol} / \mathrm{l}$ oder Anstieg der Laktatkonzentration $\Delta[L a]>1,5$

TABelle 2.5: Diagnostische Kriterien des Organversagens. 


\section{Septischer Schock}

Bei Vorliegen einer schweren Sepsis und therapierefraktärer arterieller Hypotonie wird auch der Terminus septischer Schock benutzt:

septischer Schock = schwere Sepsis + therapierefraktäre Hypotension

\subsubsection{Maligne Tumorerkrankungen}

Der Terminus maligne Tumorerkrankung wurde stellvertretend für Tumormanifestationen gebraucht, die entweder während der intensivmedizinischen Behandlung diagnostiziert oder bereits vorbestehend dokumentiert waren, unabhängig vom Stadium der Tumorerkrankung. Voraussetzung war der histopathologische Nachweis der Malignität. Das Ausgangsgewebe des Primarius wurde erfasst, ebenso das Vorliegen von Fernmetastasen. Ausschließlich lokal begrenzte Metastasen wurden nicht als Fernmetastasen gewertet. Als tumorfrei wurden diejenigen Patienten klassifiziert, deren Tumorerkrankung $\geq 5$ Jahre ohne Progression war bzw. die nach einer kurativen Therapie rezidivfrei waren. An weiteren Untergruppen wurde zwischen soliden Tumoren und hämato-onkologischen Tumoren (Hämoblastosen) unterschieden.

\subsection{Medikation}

Eine frühere oder noch anhaltende Chemotherapie bzw. eine anderweitige immunsuppressive Therapie (innerhalb der letzten 6 Wochen) wurde erfasst, dabei wurden die entsprechenden Wirkstoffe, nicht jedoch ihre Dosierung dokumentiert. Außerdem wurde die Medikation oder der Abusus von Arzneimitteln bekannter Nephrotoxität erfasst. Hierzu wurden die Originalarbeiten der Aufstellungen von Arzneimittel-bedingten Nierenschädigungen herangezogen (Schwarz 2001; Choudhury und Ahmed 2006).

\subsection{Laborbefunde}

Mit Hilfe des medizinischen Dokumentationssystems ixserv wurden die in Tab. 2.6 aufgeführten Laborbefunde ausgewertet: 


\begin{tabular}{|c|c|c|}
\hline \multicolumn{3}{|c|}{ Laborbefunde } \\
\hline \multirow{3}{*}{ Blutbild } & Hämoglobin & $13,5-17,5 \mathrm{~g} / \mathrm{dl}$ \\
\hline & Leukozyten & $4,0-11,010^{3} / \mu l$ \\
\hline & Thrombozyten & $150-30010^{3} / \mu l$ \\
\hline Gerinnung & Part. Thromboplastinzeit (PTT) & $80-130 \%$ \\
\hline \multirow{16}{*}{ Klin. Chemie } & Kalium & $3,6-4,8 \mathrm{mmol} / \mathrm{l}$ \\
\hline & Natrium & $136-145 \mathrm{mmol} / \mathrm{l}$ \\
\hline & Aspartat-Aminotransferase (AST) & $\mathrm{m} \leq 35 U / l$ \\
\hline & & $\mathrm{w} \leq 31 U / l$ \\
\hline & Alanin-Aminotransferase (ALT) & $\mathrm{m} \leq 45 U / l$ \\
\hline & & $\mathrm{w} \leq 34 U / l$ \\
\hline & $\gamma$-Glutamyltransferase $(\gamma$-GT $)$ & $\mathrm{m} \leq 12-64 U / l$ \\
\hline & & $\mathrm{w} \leq 9-36 U / l$ \\
\hline & Bilirubin, ges. & $\leq 1,2 \mathrm{mg} / \mathrm{dl}$ \\
\hline & C-reaktives Protein (CRP) & $\leq 5,0 \mathrm{mg} / \mathrm{dl}$ \\
\hline & Procalcitonin (PCT) & $\leq 0,1 \mu g / l$ \\
\hline & Kreatinin & $\mathrm{m} 0,7-1,2 \mathrm{mg} / \mathrm{dl}$ \\
\hline & & $\mathrm{w} 0,5-1,0 \mathrm{mg} / \mathrm{dl}$ \\
\hline & Harnstoff & $\mathrm{m} 8-26 \mathrm{mg} / \mathrm{dl}$ \\
\hline & & $\mathrm{w} 7-20 \mathrm{mg} / \mathrm{dl}$ \\
\hline & Glomeruläre Filtrationsrate & $>60 \mathrm{ml} / \mathrm{min} / 1.72 \mathrm{~m}^{2}$ \\
\hline Bakteriologie & Blut, Urin, Sekret, Abstrich & \\
\hline
\end{tabular}

TABelle 2.6: Übersicht der dokumentierten Laborwerte mit Angabe der Referenzintervalle (rechte Spalte). 


\subsection{Schweregrad von Erkrankungen (SAPS II)}

\section{Simplified Acute Physiology Score - SAPS II}

Das simplified acute physiology score (SAPS II) ist ein indirektes quantitatives Maßzahl-System, welches nach der Maßgabe von Punkten (scores) den physiologischen bzw. pathophysiologischen Zustand eines Patienten bewertet. Dieses System wurde im Jahre 1993 etabliert, um eine Basis für die Vergleichbarkeit von Patienten-Gruppen mit unterschiedlichen Erkrankungen und unterschiedlichem Schweregrad der Erkrankungen in wissenschaftlich-klinischen Untersuchungen zu gewährleisten (Le Gall et al. 1993). Hierbei wird das Ausmaß der Abweichung vom physiologischen Normalzustand, bezogen auf allgemeine Variablen, Laborwerte sowie andere Umstände (insgesamt 15 Variablen) mit unterschiedlich gewichteten Punktzahlen bewertet. Die kumulative Punktsumme ist eine indirekte Kenngröße für den Schweregrad einer Erkrankung und ist grundsätzlich von der Art der Erkrankung(en) unabhängig. Die Variablen, die zur Berechnung der Gesamt-Punktzahl des SAPS II herangezogen werden, sind in Tab. 2.7 und Tab. 2.8 zusammengefasst. Die Minimum - Maximum Punkteskala beträgt 0 - 163; eine höhere Punktzahl bedeutet eine schwerwiegendere Erkrankung. Die SAPS II-Punktwerte werden erstmals $24 h$ nach der Patientenaufnahme ermittelt und im Verlauf der Behandlung täglich neu berechnet.

Die Glasgow-Koma-Skala (Glagow Coma Scale, GCS) ist Bestandteil des SAPS IIBewertungssystems. Die GCS-Skala ist eine einfache Bewertungsskala für die Bewusstseinslage eines Patienten (Teasdale und Jennet 1974). Es werden 3 Kriterien betrachtet: Augenöffnung, verbale Kommunikation und motorische Reaktionen. Die Punktebewertung im Bereich von 3-15 erfolgt in umgekehrter Weise nach Ausmaß der BewusstseinsStörung: $15=$ volles Bewusstsein, $\geq 8=$ schwere Bewusstseins-Einschränkung, $3=$ tiefes Koma.

Aus den verfügbaren Patientendokumentationen wurden die SAPS II-Angaben entnommen bzw. fehlende Angaben durch Neuberechnung ergänzt. Patienten mit Verbrennungen sowie Patienten mit herzchirurgischen Eingriffen wurden in der vorliegenden Untersuchung ausgenommen.

Grundsätzlich ist bei dem Bewertungssystem SAPS II zu beachten, dass es sich in der Typologie um ein abstraktes, approximativ und empirisches Patienten-KlassifikationsSystem handelt, dessen inhaltliche Validität nicht zweifelsfrei und abschließend dargestellt ist. Es ist der Natur nach Ausdruck eines stark vereinfachenden, kollektiven und pauschalen Bemessungssystems und stellt lediglich eine Orientierung dar. Die Klassifizierung nach Schweregraden entsprechend den SAPS II-Kriterien hat keine Bedeutung im Hinblick auf diagnostische oder therapeutische Konsequenzen und stellt für den 
Patienten keinerlei unmittelbaren oder mittelbaren Nutzen dar. Sie dient lediglich der wissenschaftlichen a posteriori-Betrachtung von Patientenkollektiven hinsichtlich ihrer Vergleichbarkeit.

\subsection{Statistische Auswertung}

Kontinuierliche Variable sind als Mittelwerte \pm SD oder Median (25 - 75\% Interquartilsabstand) angegeben. Kategoriale Variable sind als absolute Fraktion oder relativ (\%) angegeben. Unterschiede zwischen 2 Gruppen wurden durch Chi-Quadrat und Exakten Fischer-Test statistisch ausgewertet. Univariate und multivariate Regressionsanalysen wurden benutzt, um Auswirkungen unabhängiger Variablen bezüglich abhängiger Variablen zu testen. Unterschiede zwischen 3 oder mehr Gruppen wurden durch Varianzanalyse (ANOVA) analysiert. Es wurden die üblichen Signifikanzgrenzen gewählt: $\left.p>0.05(\mathrm{NS}) ; p<0.05\left(^{*}\right) ; p<0.01{ }^{(* *}\right) ; p<0.001$ (***) $^{*}$. 


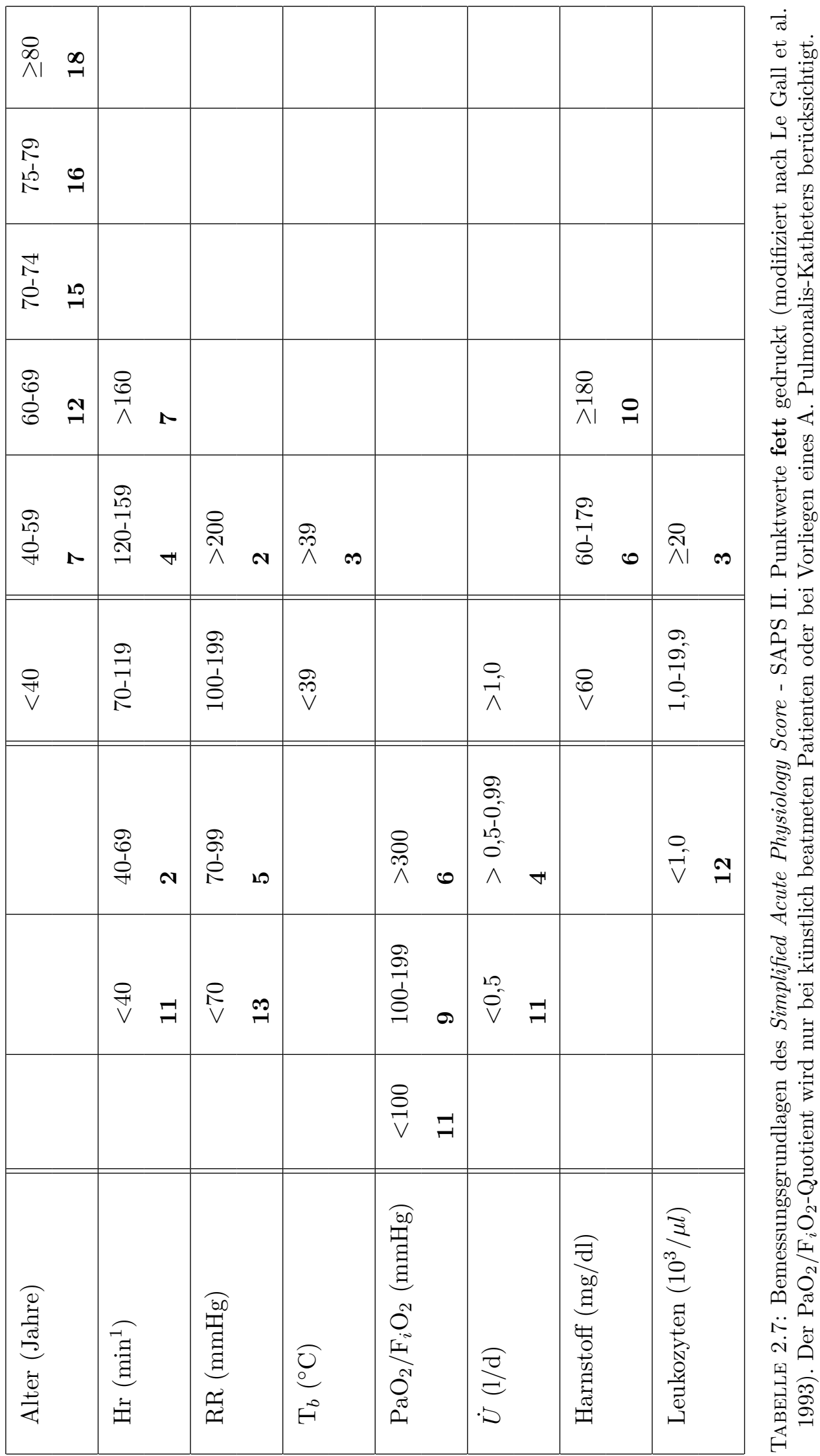




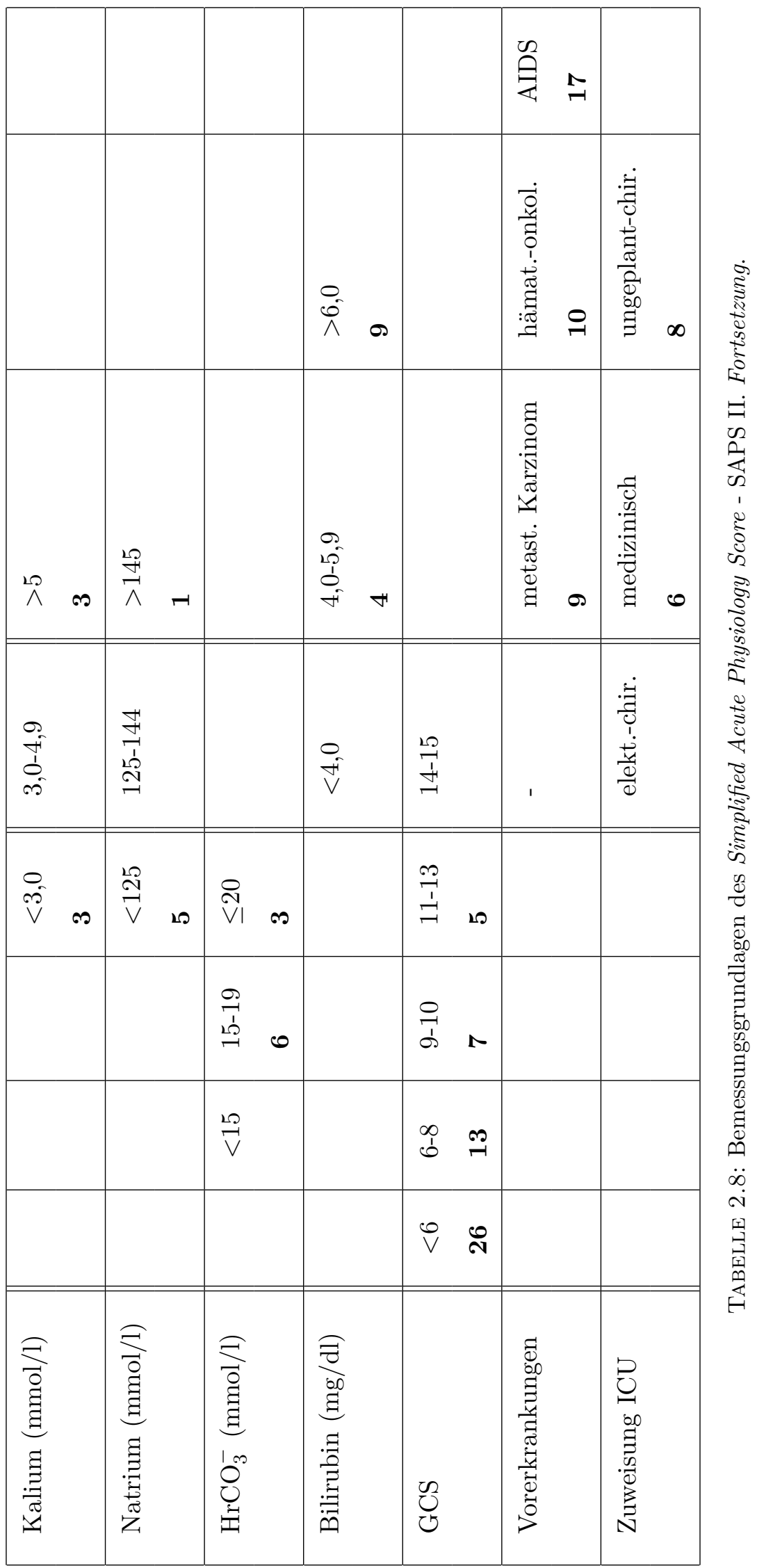




\section{Kapitel 3}

\section{Ergebnisse}

\subsection{Patientenkollektiv - Allgemeine Merkmale}

In der vorliegenden Untersuchung wurden alle Patienten erfaßt, die über einen Zeitraum von 3 Jahren vom 01.01.2009 - 31.12.2011 auf der nephrologischen Intensivstation (ICU) des Universitätskrankenhauses Göttingen (UMG) behandelt wurden $(N=1017)$. In der weiteren Analyse wurden aus diesem Patientenkollektiv 18 Patienten ausgeschlossen, deren Daten unvollständig waren, ebenso wie 2 Patienten, die zum Aufnahmezeitpunkt jünger als 18 Jahre waren. Die Gesamtzahl des in dieser Untersuchung betrachteten Patientenkollektivs war daher auf $N=997$ ( $m=594, w=403)$ reduziert. Das mittlere Alter aller Patienten lag bei $65 \pm 16$ Jahren $(m 66 \pm 18 y, w 64 \pm 15 y)$. Der Altersschwerpunkt der Patienten lag daher im fortgeschrittenen Lebensalter. Die mittlere Behandlungsdauer aller Patienten betrug 7,4 8,2 Tage. Die Behandlungsdauer der Überlebenden und der Verstorbenen ist in Abb. 3.1 zusammengefaßt.

Die Auswertung der Patientendaten ist ausschließlich auf den 3-Jahres-Zeitraum der Behandlung auf der ICU beschränkt. Der weitere Krankheits- und Behandlungsverlauf konnte wegen nicht verfügbarer Patientendokumentation, z.B. durch Verlegung in ein auswärtiges Krankenhaus, nicht weiter verfolgt werden. Eine Übersicht der Behandlungszahlen im Zeitraum der Untersuchung ist in Tab. 3.1 dargestellt. Danach waren die Patientenzahlen im Jahreszeitraum 2009 - 2010 um 22, 4\%, im Jahreszeitraum 2010 - 2011 um 14, $9 \%$ steigend. Die relativen Zahlen zu Prävalenz und Mortalität der Haupterkrankungen (AKI, Sepsis und Malignom) zeigen aber keine eindeutige Tendenz oder auffällige Abweichungen.

In der folgenden Analyse werden zunächst die allgemeinen Merkmale des Patientenkollektivs behandelt, eine differenzierte Betrachtung der verschiedenen Krankheitszustände und ihre Kombinationen (Komorbidität) folgt in den weiteren Abschnitten. 


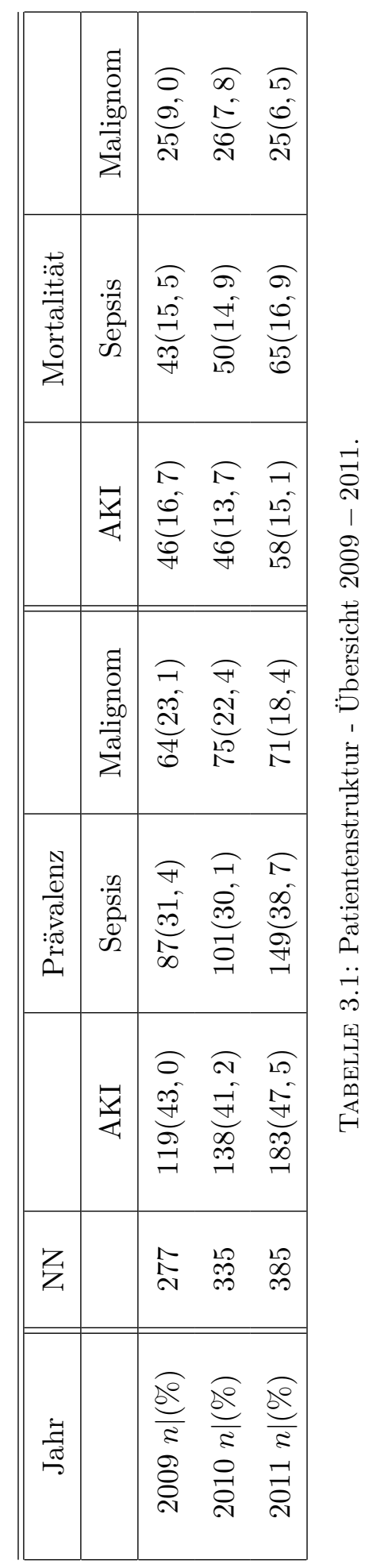



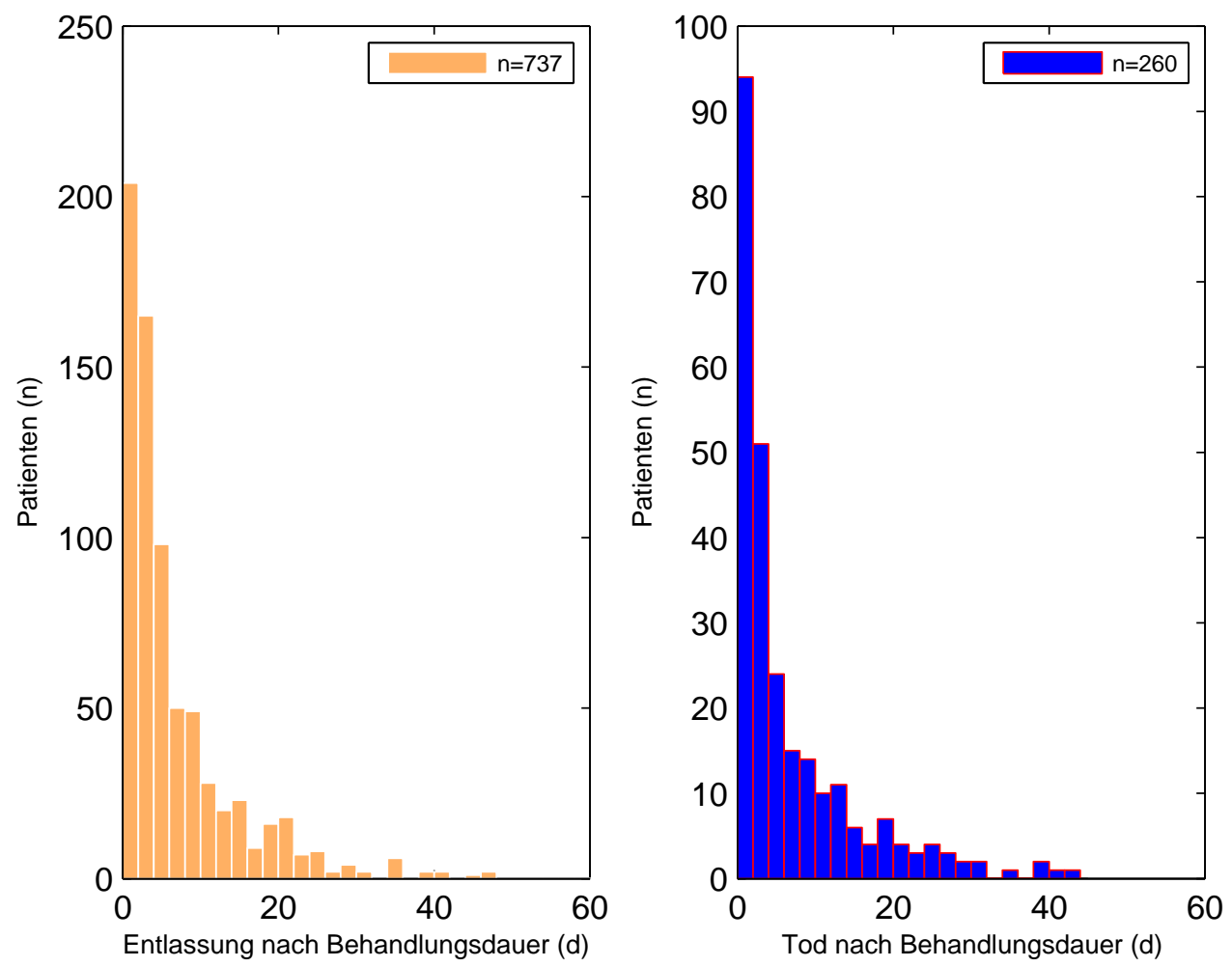

AbBildung 3.1: Behandlungsdauer der Überlebenden (linke Abb.) und der Verstorbenen (rechte Abb.).

Bei der Darstellung der Ergebnisse handelt es sich überwiegend um die Betrachtung von Fallzahlen, die als Teilmengen einer Gesamtmenge angegeben sind. Die Teilmengen sind entweder als dimensionslose absolute Fraktion, bezogen auf die Gesamtmenge $(1,0=$ $\left.\sum_{n / N}^{N}\right)$ oder als relative Fraktion in Prozent $\left(1,0 * 100=\sum_{n / N}^{N}[\%]\right)$ angegeben.

In der Analyse bilden AKI, Sepsis und Malignom als selektive Krankheitsbilder den Schwerpunkt der Betrachtung. Die Untersuchung ist auf die Frage der Morbidität gerichtet, die den Überbegriff für die Prävalenz einer Erkrankung darstellt. Die Häufigkeit des Auftretens einer Erkrankung in einem Patientenkollektiv wird mit dem Begriff Prävalenz bezeichnet. Die Prävalenz bezeichnet die Anzahl der zum Untersuchungszeitraum erkrankten Patienten, d.h. die Anzahl der in die Untersuchung einbezogenen Individuen. Dagegen wird mit dem Begriff Inzidenz die Häufigkeit einer neu aufgetretenen Erkrankung bezeichnet. Da es sich beim AKI und der Sepsis um Erkrankungen handelt, die sich innerhalb eines kurzen Zeitraums (Stunden, Tage) entwickeln - in der Regel akute (neue) Komplikationen eines andersartigen bestehenden schweren Grundleidens darstellen - und in dieser Untersuchung nur der Behandlungszeitraum auf der ICU betrachtet 
wird, können die Begriffe Prävalenz und Inzidenz bei diesen Erkrankungszuständen synonym verwendet werden. Bei malignen Tumorerkrankungen ist diese begriffliche Äquivalenz nicht gegeben.

Die Angaben zu Todesfällen im Patientenkollektiv - oder bei nach Merkmalen klassifizierten Untergruppen - werden durch die Begriffe Mortalität und Mortalitätsrate gekennzeichnet. Hierbei wird mit Mortalität allein die dimensionslose Häufigkeit des Todeseintritts bezeichnet. Eine Rate ist immer mit einem zeitlichen Bezug auf das Zeitintervall der Betrachtung verbunden. In der vorliegenden Untersuchung werden nur die Todesfälle betrachtet, die im relativ kurzen Zeitraum der Behandlung auf der ICU (im Mittel 7,4 \pm , 2 Tage) eingetreten waren. Somit können in diesem Fall die Begriffe Mortalität und Mortalitätsrate synonym verwendet werden.

\subsection{Erkrankungen und Diagnosen}

Bei 78 Patienten $(7,8 \%)$ erfolgte die Aufnahme aus postoperativ chirurgischer Indikation, bei 919 Patienten $(92,2 \%)$ war eine internistische Indikation gegeben.

Eine Übersicht der in dieser Arbeit behandelten Schwerpunktdiagnosen AKI, Sepsis und Malignom sowie der Diagnosen von Grund- bzw. Begleiterkrankungen ist in Abb. 3.2 zusammengefaßt. Die Begleiterkrankungen sind nach übergeordneten kollektiven Organerkrankungen bzw. Erkrankungszuständen subsummiert.

Es wurden für die 3 Haupterkrankungen (AKI, Sepsis und Malignom) in den jeweils bezüglich eines Merkmals korrespondierenden Patientengruppen, z.B. Vorliegen eines AKI $[$ AKI (+)] bzw. Fehlens eines AKI [AKI (-)], die Fraktionen der jeweiligen Begleiterkrankungen berechnet und als Differenzfraktionen (Ordinate) dargestellt. Die Differenzfraktion kann positive oder negative Werte annehmen. Positive Zahlenwerte bedeuten, dass eine Begleiterkrankung in der Patientengruppe mit positivem Merkmal, z.B. [AKI (+)] überwiegt, negative Zahlenwerte bedeuten, dass eine Begleiterkrankung in der Patientengruppe mit negativem Merkmal, z.B. [AKI (-)] überwiegt.

Bei Patienten mit AKI überwiegen Pneumonie und Immunsuppression, bei Patienten mit Sepsis überwiegen ausnahmslos alle verzeichneten Begleiterkrankungen, bei Patienten mit Malignom imponieren Pneumonie und Immunsuppression. Bemerkenswert ist die hohe Prävalenz einer Pneumonie bei Patienten mit Sepsis; keine wesentliche Prävalenz bestand bei vorausbestehender chronischer Niereninsuffizienz. Bei Patienten mit Malignom ist die Prävalenz von Pneumonie und - erwartungsgemäß aus therapeutischen 


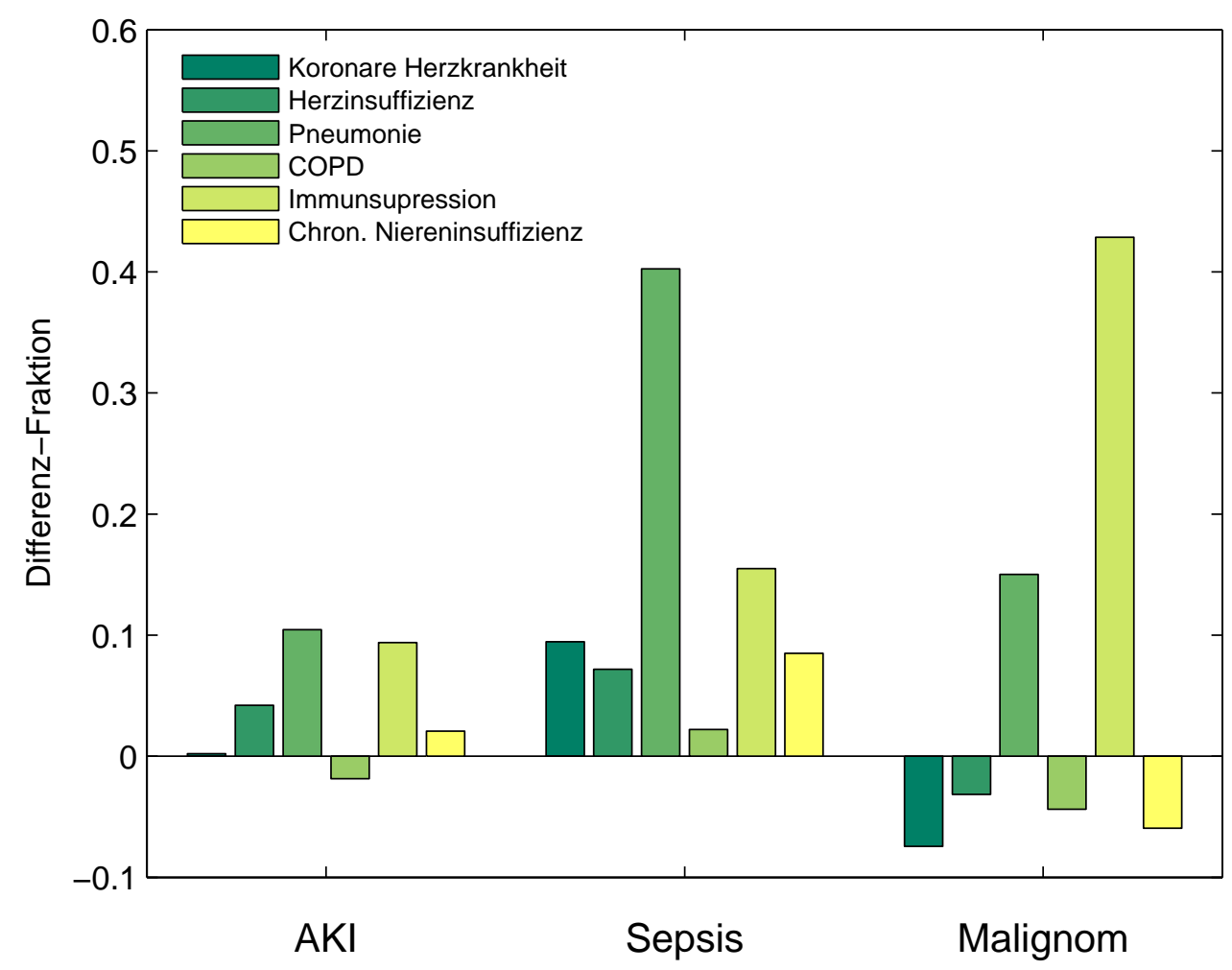

ABBiLdung 3.2: Patientenstruktur - Hauptdiagnosen und Begleiterkrankungen. Erläuterungen im Text.

Gründen - die der Immunsuppression dominierend. Die Übersicht besagt in allgemeiner Formulierung, dass in den korrespondierenden Patienten-Vergleichs-Kollektiven die Prävalenzen der Begleiterkrankungen sehr inhomogen verteilt waren.

\subsection{Mortalität und Todesursachen}

Die Gesamtmortalität der im Betrachtungszeitraum auf der ICU behandelten Patienten betrug 26,1\% $(n=260)$. Aus der Gruppe post-operativ chirurgischer Patienten verstarben 16,7\%, aus der Gruppe internistischer Patienten 26,9\%.

Die Übersicht zu Prävalenz und Mortalität bezogen auf die Hauptdiagnosen AKI, Sepsis und Malignom sind in Abb. 3.3 dargestellt.

Hieraus ergibt sich, dass die Behandlungsschwerpunkte der ICU in numerisch absteigender Folge auf die Haupterkrankungen konzentriert waren: AKI $(44,6 \%)>$ Sepsis $(34,1 \%)>$ Malignom $(21,3 \%)$. 

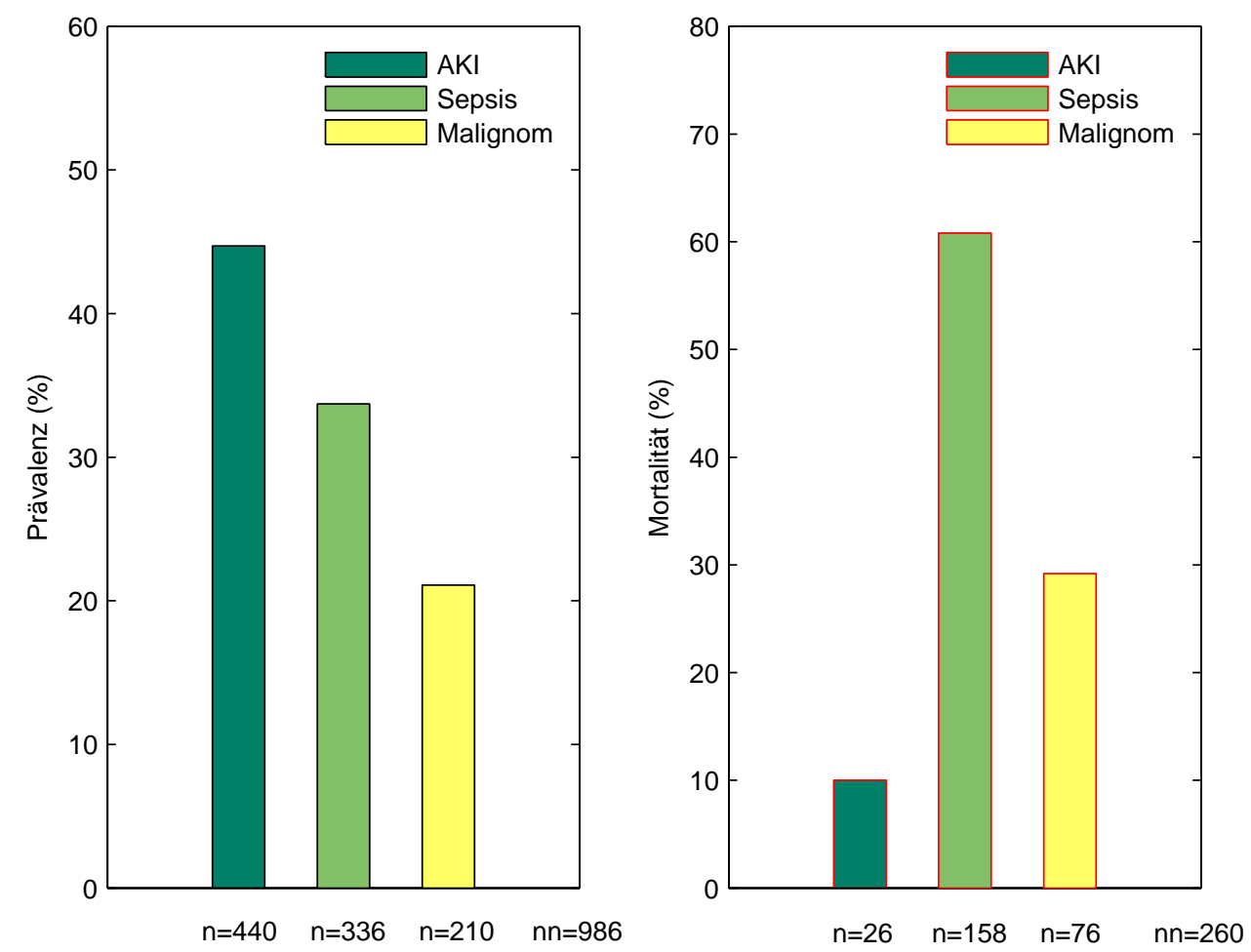

AbBildung 3.3: Prävalenz (linke Abb.) und Mortalität (rechte Abb.) der Haupterkrankungen im Patientenkollektiv.

Die Mortalität war bei Patienten mit Sepsis am höchsten (60,8\%), gefolgt von Patienten mit Malignom (29,2\%). Bei der Mortalität der AKI-Patienten (10,0\%) ist zu beachten, dass aus dieser Angabe kein fälschlich kausaler Zusammenhang zwischen AKI und Mortalität hergestellt wird. Die Zahlenangabe von 10,0\% unter der Rubrik AKI besagt lediglich, dass bei diesen Patienten die Behandlung eines AKI unter intensivmedizinisch optimaler Therapie erfolgte, die Mortalität aber aus anderen Krankheitsursachen gegeben war, die außerhalb der Haupterkrankungen der Gruppen Sepsis und Malignom lagen.

Die Mortalität im Patientenkollektiv ist in anderer Form in Abb. 3.4 dargestellt. Die Abb. 3.4 (links) zeigt das in aufsteigend geordneter Form kumulative Histogramm (Häufigkeitsdichte) aus Patientenzahl $(n=440)$ und Überlebenszeit $(1-44$ Tage), die der Behandlungsdauer auf der ICU entspricht. In Abb. 3.4 (rechts) ist die daraus berechnete empirische Kerndichteschätzung (Schätzung der Wahrscheinlichkeitsdichteverteilung, 

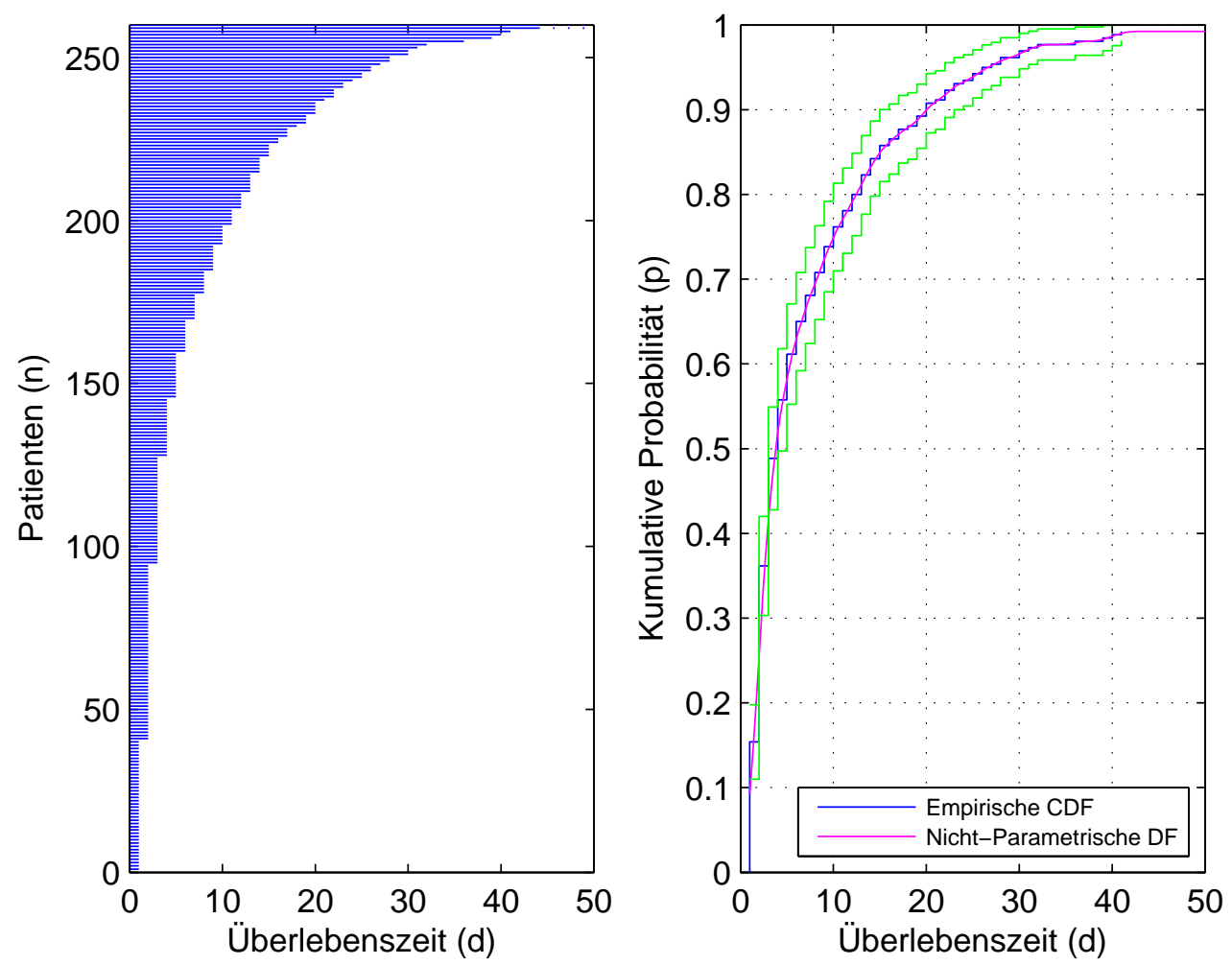

AbBildung 3.4: Mortalität im Patientenkollektiv. Linke Abb. Kumulative Darstellung der Mortalität aufsteigend nach Patientenzahl und Überlebenszeit geordnet. Rechte Abb. Wahrscheinlichkeitsdichtefunktion (Empirische kumulative Dichtefunktion (CDF, blau) mit 95\% Vertrauensgrenzen (grün) und berechneter stetiger nicht-parametrischer Dichtefunktion (magenta). Weitere Erläuterungen im Text.

cumulative probability density function, CDF) dargestellt, die den typischen treppenförmigen Verlauf (blau) mit 95\% Vertrauensgrenzen (grün) annimmt. Die nach der nichtparametrischen Kernel-Dichte-Methode (Kerndichteschätzung, kernel density estimation, KDE) berechnete kontinuierliche, stetige Dichtefunktion (magenta) zeigt eine sehr gute Anpassung an die empirische kumulative Probabilitätsdichtefunktion. Auf eine detaillierte Beschreibung der mathematischen Grundlagen wird an dieser Stelle verzichtet. Aus dem Verlauf der stetigen Dichtefunktion kann die Wahrscheinlichkeit entnommen werden, dass bei der gegebenen Typologie der Erkrankungen des Patientenkollektivs $50 \%$ der Patienten $(p=0,5)$ nach 3,8 Tagen versterben, 75\% der Patienten werden nach 10,1 Tagen Behandlungsdauer auf der ICU versterben.

Die Zahlenangaben zur Mortalität im Patientenkollektiv geben keinen Aufschuss über die Ursache der Mortalität. Die Todesursachen sind nach vorherrschenden Organ- bzw. Funktionssystemen geordnet in Abb. 3.5 dargestellt. Die häufigste Todesursache war ein 


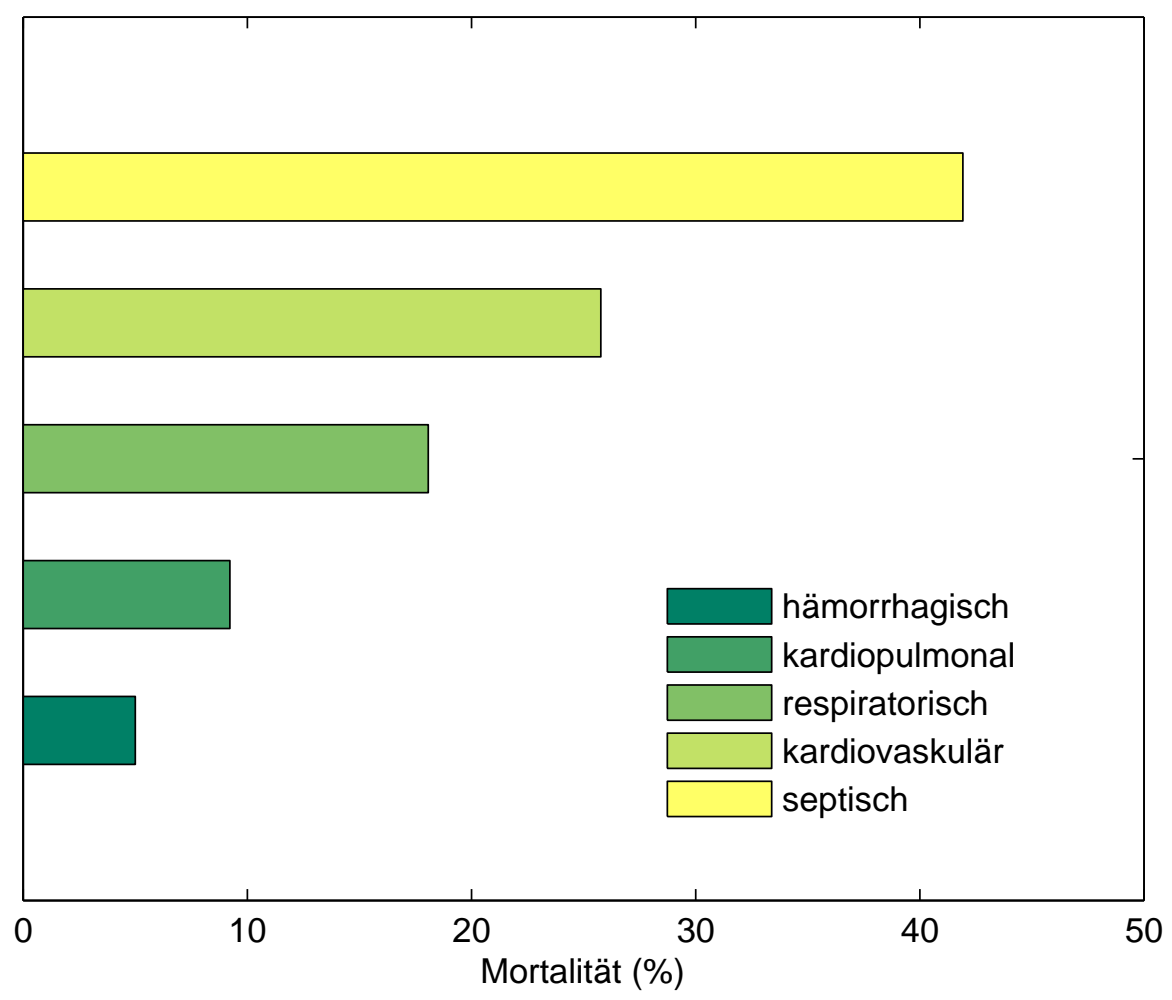

AbBildung 3.5: Todesursachen im Patientenkollektiv.

Multiorganversagen (multiorgan dysfunction syndrome, MODF) im Rahmen einer septischen Erkrankung (41,9\%). An zweiter Stelle folgten kardiovaskuläre Ursachen mit kardiogenem Schock $(25,8 \%)$. Respiratorische $(18,1 \%)$ und kardiopulmonale $(9,1 \%)$ Gründe waren ursächlich für ein Versterben. Eine dekompensierte Leberzirrhose bei 7 Patienten und unkontrollierbare innere Blutungen bei 6 Patienten führten zum Tod durch hämorrhagischen Schock $(5,0 \%)$.

\subsection{SAPS II - Klassifizierung des Schweregrades von Er- krankungen}

Das simplified acute physiology score (SAPS II) ist ein indirektes quantitatives Maßzahl-System, wonach nach der Maßgabe von Punkten (scores) der physiologische bzw. pathophysiologische Zustand eines Patienten bemessen werden kann (s. Kap. 2.8). Das Bewertungssystem ist grundsätzlich unabhängig von der Morbidität, d.h. unabhängig von der Art der vorliegenden Erkrankung(en) und deren Komplikationen. Die Bewertungspunktzahlen sind als Funktion der Behandlungsdauer in Abb. 3.6 dargestellt. Es 
wurden vergleichsweise der Mittelwert über die Dauer der Behandlung auf der ICU $\left(\mathrm{SAPS}_{\text {mean }}\right)$ und der singuläre Maximalwert $\left(\mathrm{SAPS}_{\max }\right)$ betrachtet.
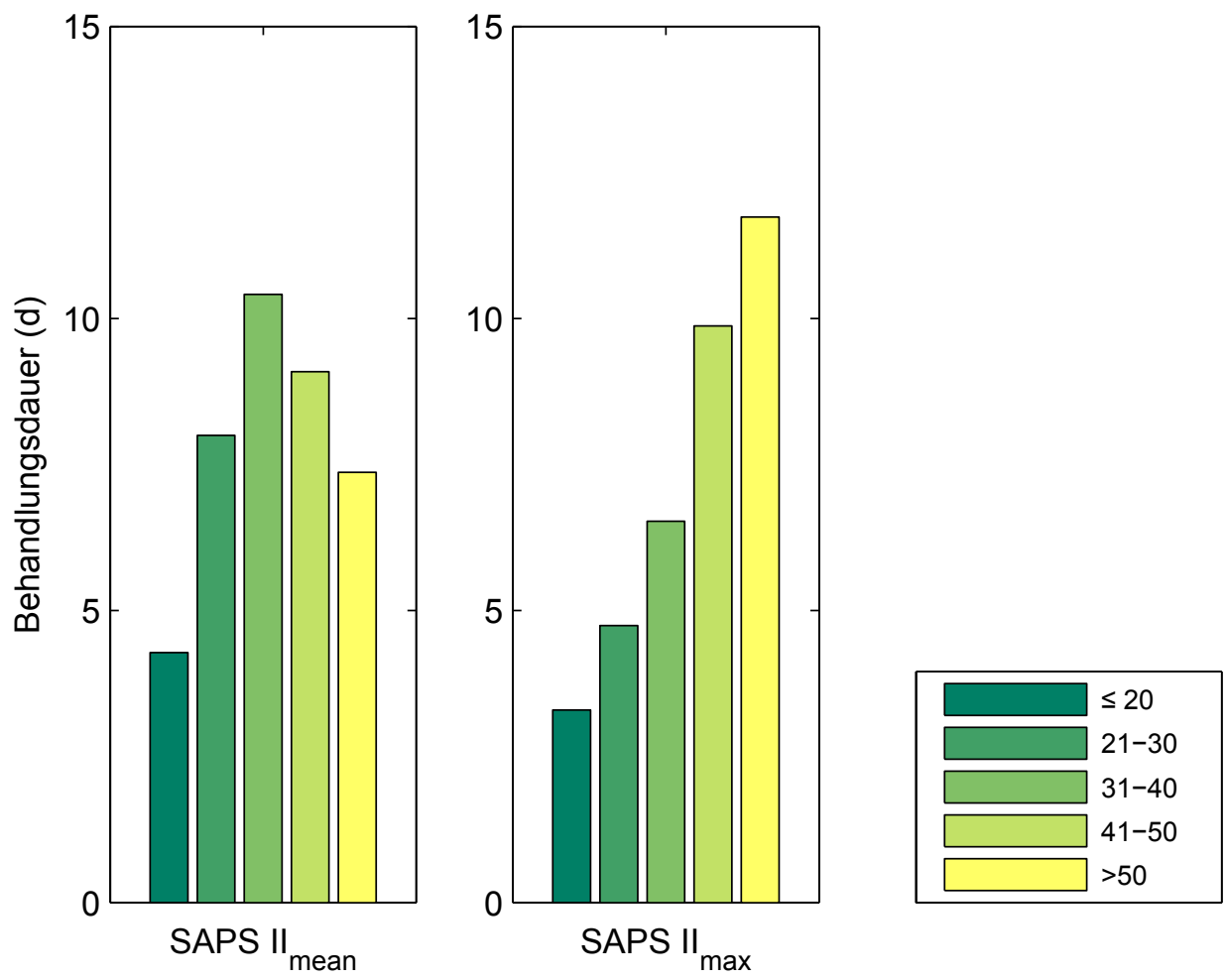

AbBILDUnG 3.6: Schweregrad von Erkrankungen - SAPS II.

Dargestellt sind die Punktwerte des SAPS II ( SAPS $\left._{\text {mean }}\right)$ in gruppierter Form. Die Behandlungsdauer nahm mit zunehmendem Schweregrad der Erkrankungen zu und erreichte ein Maximum im Bereich von 31 - 40 Punkten. Dies entspricht dem Schweregrad mittelschwerer Erkrankungen. Mit weiter zunehmendem Schweregrad nahm die Behandlungsdauer wieder ab, was auf den Umstand zurückzuführen ist, dass Patienten mit schweren bis sehr schweren Erkrankungen nach kürzeren Behandlungszeiten verstar-

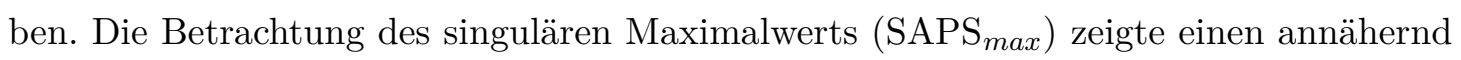
proportionalen Zusammenhang mit der Behandlungsdauer.

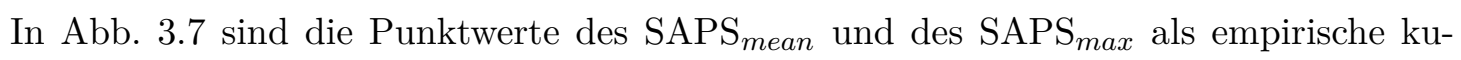
mulative Dichtefunktionen dargestellt (analog zur Darstellung der Überlebenszeit im Patientenkollektiv in Abb. 3.4 rechts). Der Kurvenverlauf beider Kurven ist ähnlich,

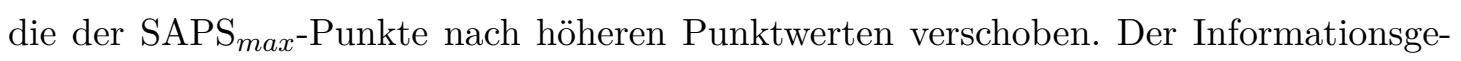
halt bei Erhebung der Punktwerte von SAPS mean $_{\text {oder SAPS }}$ max ist, abgesehen von quantitativen Unterschieden, grundsätzlich der Gleiche, sodass auf die Erfassung von SAPS $_{\text {max }}$-Punktwerten verzichtet werden kann. 


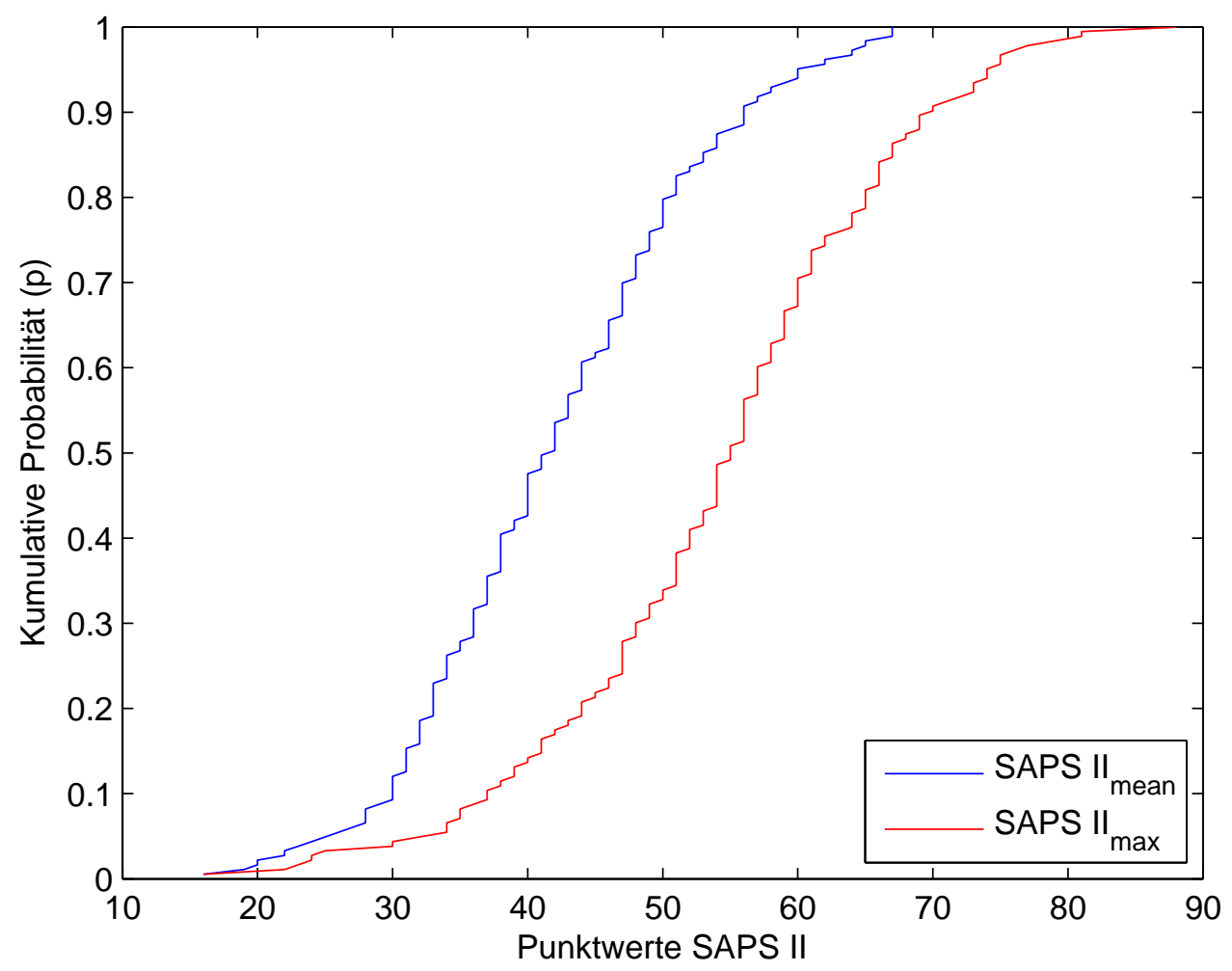

AbBILDUng 3.7: SAPS II - Empirische kumulative Dichtefunktion.

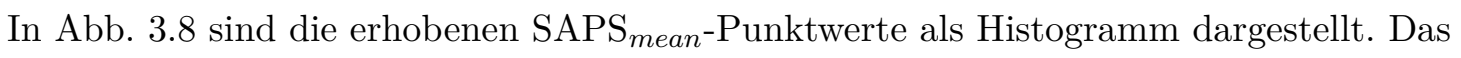
Histogramm stellt eine skalierte Form der empirischen Wahrscheinlichkeitsdichtefunktion dar. Eine Anpassung eines univariaten Datensatzes in Form eines Histogramms an eine Verteilungsfunktion ist mit theoretischen Problemen bei Anwendung der Methode der kleinsten Quadrate verbunden. Eine effiziente Methode der Modellanpassung stellt die Maximum-Likelihood-Methode zur Anpassung einer Weibull-Verteilung an den Datensatz dar. Die Abb. 3.8 zeigt das auf den Wert von 1,0 integrierte Histogramm mit der an die Daten angepassten überlagerten Wahrscheinlichkeitsdichtefunktion. Zur Klarheit sei darauf hingewiesen, dass es sich hier nicht um eine Kurvenanpassung eines univariaten Datensatzes im üblichen Sinne handelt, sondern vielmehr um eine Anpassung an eine Verteilung, wobei das Modell eine normalisierte Probabilitätsfunktion darstellt. Das Maximum der Verteilungsfunktion liegt bei 35 SAPS II-Punktwerten. Die Einbeziehung dieser Berechnungen erfolgt hier, um eine geeignete Form der Darstellung von Schweregraden von Erkrankungen nach dem SAPS II-Klassifizierungssystem aufzuzeigen, welches bei vergleichenden retrospektiven Analysen zur Anwendung kommen könnte. 


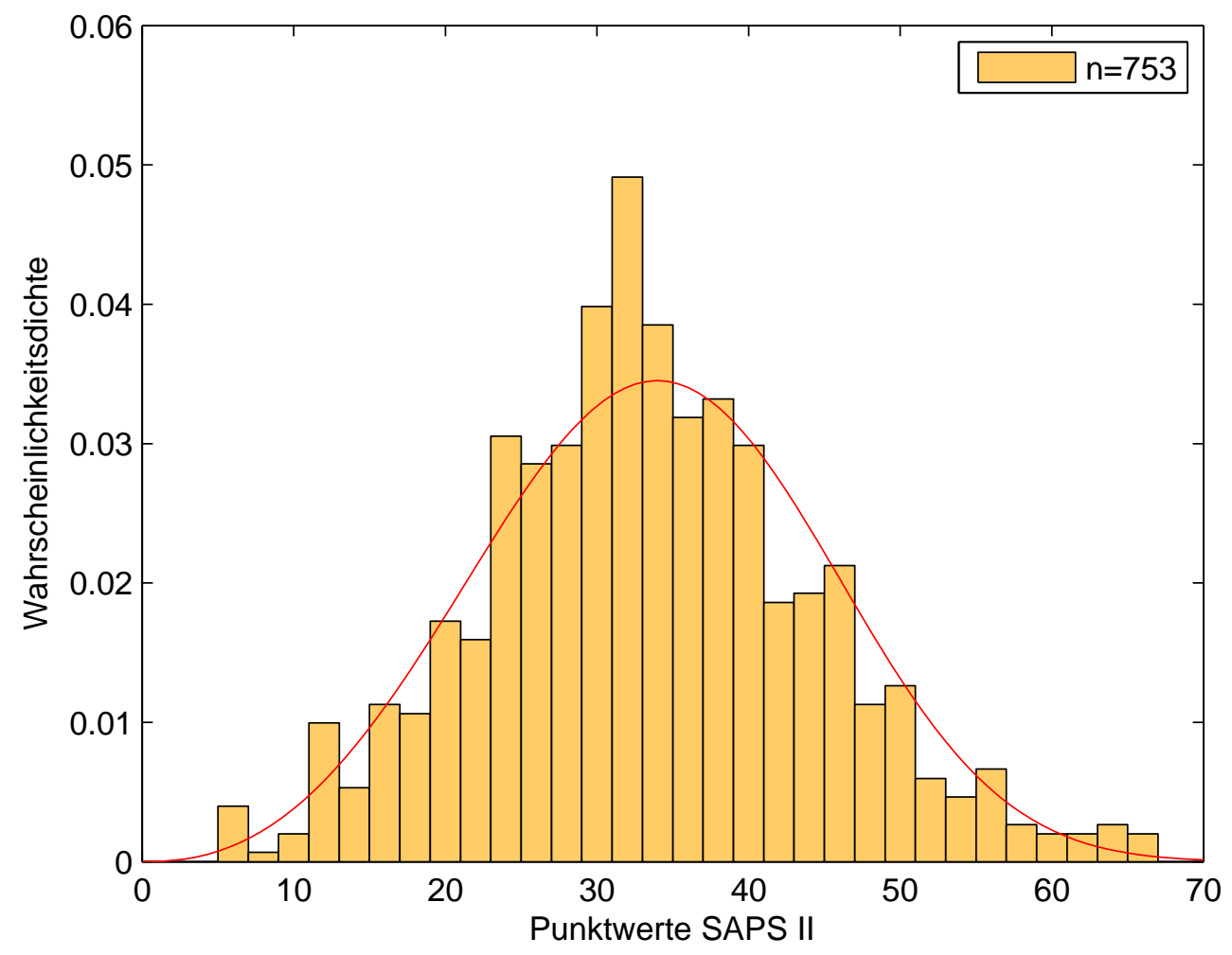

ABBILDUng 3.8: SAPS II - Histogramm und Verteilungsfunktion.

\subsection{Faktoren des Behandlungserfolgs}

Unter den Faktoren zur Beurteilung des Behandlungserfolgs (outcome) sind in Tab. 3.2 ausgewählte Merkmale (Erkrankungen und andere Umstände) zusammengestellt, die die Wahrscheinlichkeit eines Todeseintritts begünstigen. Es handelt sich um eine Risikoanalyse der überlebenden (positiver Behandlungserfolg) und verstorbenen (negativer Behandlungserfolg) Patienten insoweit, als trotz optimaler intensivmedizinischer Therapie ein Fortschreiten des Krankheitsverlaufs mit Eintritt des Todes nicht aufgehalten werden konnte. Aufgeführt ist das Quotenverhältnis (odds ratio, R), das eine statistische Risikobetrachtung mit Ausgabe einer assoziativen Maßzahl über die Stärke eines Zusammenhangs von zwei Merkmalen darstellt. Es handelt sich um ein Effektmaß für dichotome Daten und beschreibt das Risiko für ein Ereignis. Die odds ratio, auch als relatives Risiko bezeichnet, besagt, um wievielmal häufiger eine Erkrankung mit einem bestimmten Merkmal oder einer Komplikation im Vergleich zu einer Kontrollgruppe auftritt. Das dimensionslose Quotenverhältnis bezeichnet ein Chancenverhältnis und gibt an, mit welcher Stärke ein vermuteter Risikofaktor mit einer bestimmten Erkrankung zusammenhängt. Die odds ratio stellt keinen Beweis für einen tatsächlichen kausalen 


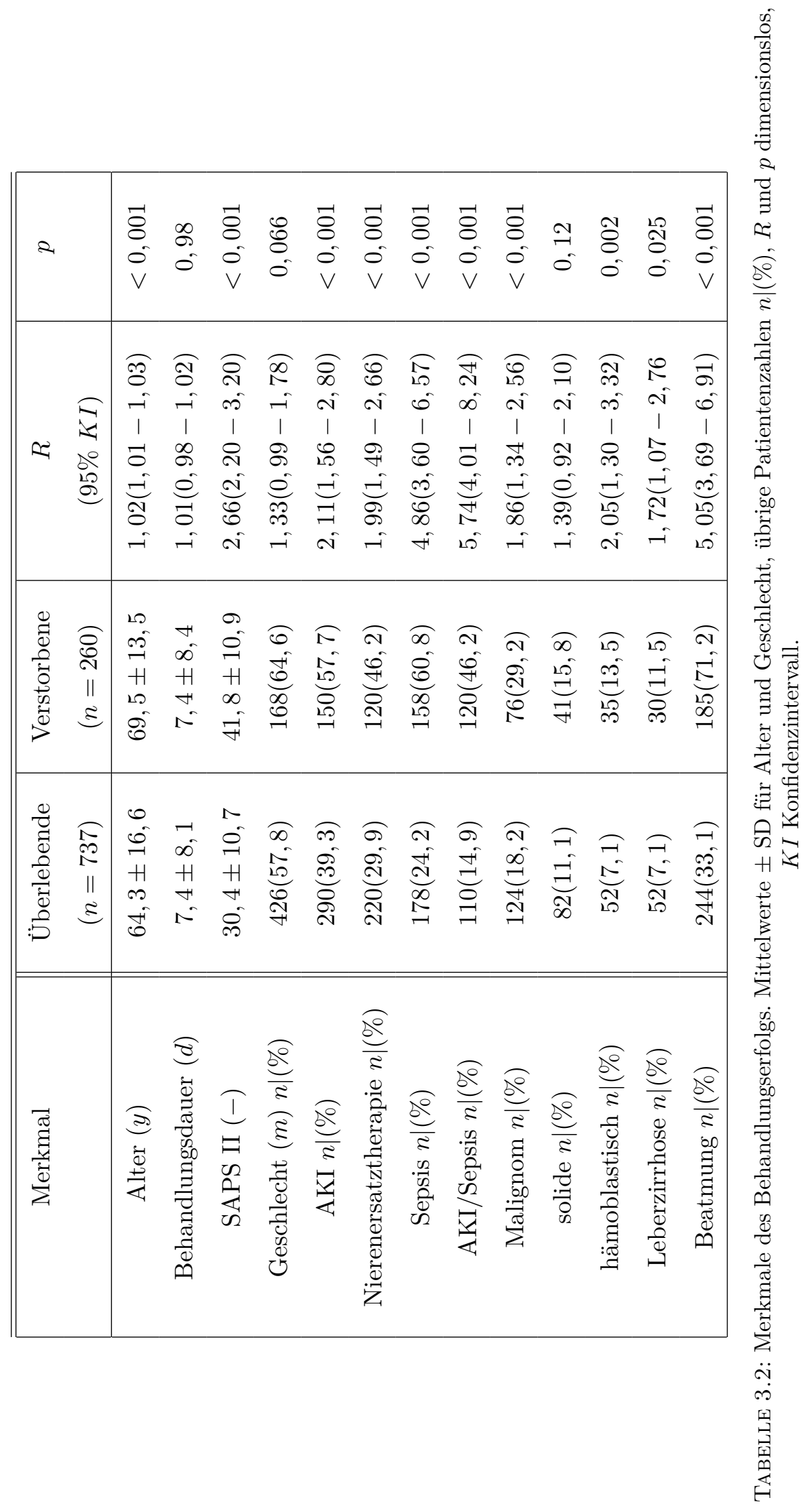


Zusammenhang zwischen Eigenschaft/Merkmal und Erkrankung dar, sie ist ein rein deskriptives Maß einer beobachteten Häufigkeit und gibt lediglich einen Hinweis auf eine mögliche Ursache des Zusammenhangs einer Grunderkrankung mit einer Folgeerkrankung oder Sekundärleiden.

In dieser Untersuchung werden die auf der ICU überlebenden und verstorbenen Patienten mit dem potentiellen Risikofaktor Tod miteinander verglichen. Das Quotenverhältnis kann Werte zwischen 0 und $\infty$ annehmen. Ein Wert von 1,0 bedeutet ein gleiches Quotenverhältnis. Beim Vergleich der Überlebenden mit den Verstorbenen bezeichnen $R$-Werte $>1,0$ ein $R$-fach gesteigertes Risiko (gleichbedeutend mit Wahrscheinlichkeit oder Chance) des Todeseintritts.

Aus den in Tab. 3.2 ausgewiesenen Quotenverhältnissen $(R)$ ergeben sich die Merkmale und Begleitumstände, die mit einer erhöhten Mortalität im Patientenkollektiv assoziiert waren. AKI, Nierenersatztherapie und maligne Neoplasien $(R \sim 2)$ bedeuten ein erhöhtes Mortalitätsrisiko. Ein $\sim 5$-fach erhöhtes Risiko für den Todeseintritt ergibt sich bei Sepsis $(R=4,86)$, insbesondere in Kombination von AKI und Sepsis $(R=5,74)$, sowie bei der Notwendigkeit einer künstlichen Beatmung $(R=5,05)$. Der Schweregrad der Erkrankung nach den SAPS II-Kriterien (s. Kap. 2.8) war bei den Verstorbenen signifikant erhöht.

\subsection{Akutes Nierenversagen}

Die Prävalenz des AKI $(n=440)$ im Patientenkollektiv lag bei 44,1\%. Zum Zeitpunkt der Aufnahme auf die ICU hatten 315 (71,6\%) Patienten ein AKI, 125 (28,4\%) Patienten entwickelten ein AKI während der Behandlung auf der ICU. Da es sich bei letzteren um Neu-Erkrankungen während der Behandlung auf der ICU handelt, muss in diesen Fällen von Inzidenz bzw. Inzidenzrate gesprochen werden. Eine akute Verschlechterung der Nierenfunktion auf dem Boden einer vorbestehenden Nierenerkrankung war bei 130 $(29,5 \%)$ Patienten der Grund für die Behandlung auf der ICU. Eine Übersicht über die Untergruppe der Patienten mit AKI gibt die Abb. 3.9.

Die häufigste Ursache des AKI (74,7\%) war prärenal infolge von Hypovolämie oder Hypotonie (kardiorenales Syndrom, Schock, hepatorenales Syndrom) oder schwere Sepsis mit Organversagen (septischer Schock). Eine intrarenale Pathophysiologie der Funktionsstörung lag in 17,5\% der Fälle vor, hervorgerufen durch exogene Schädigungen (Chemotherapeutika, Röntgenkontrastmittel) oder endogene Noxen (Hämolyse, Rhabdomyolyse) sowie thrombotische Mikroangiopathien und/oder strukturelle (Nierentumor) bzw. funktionelle entzündliche Ursachen (Glomerulonephritis, tubulointerstitielle 


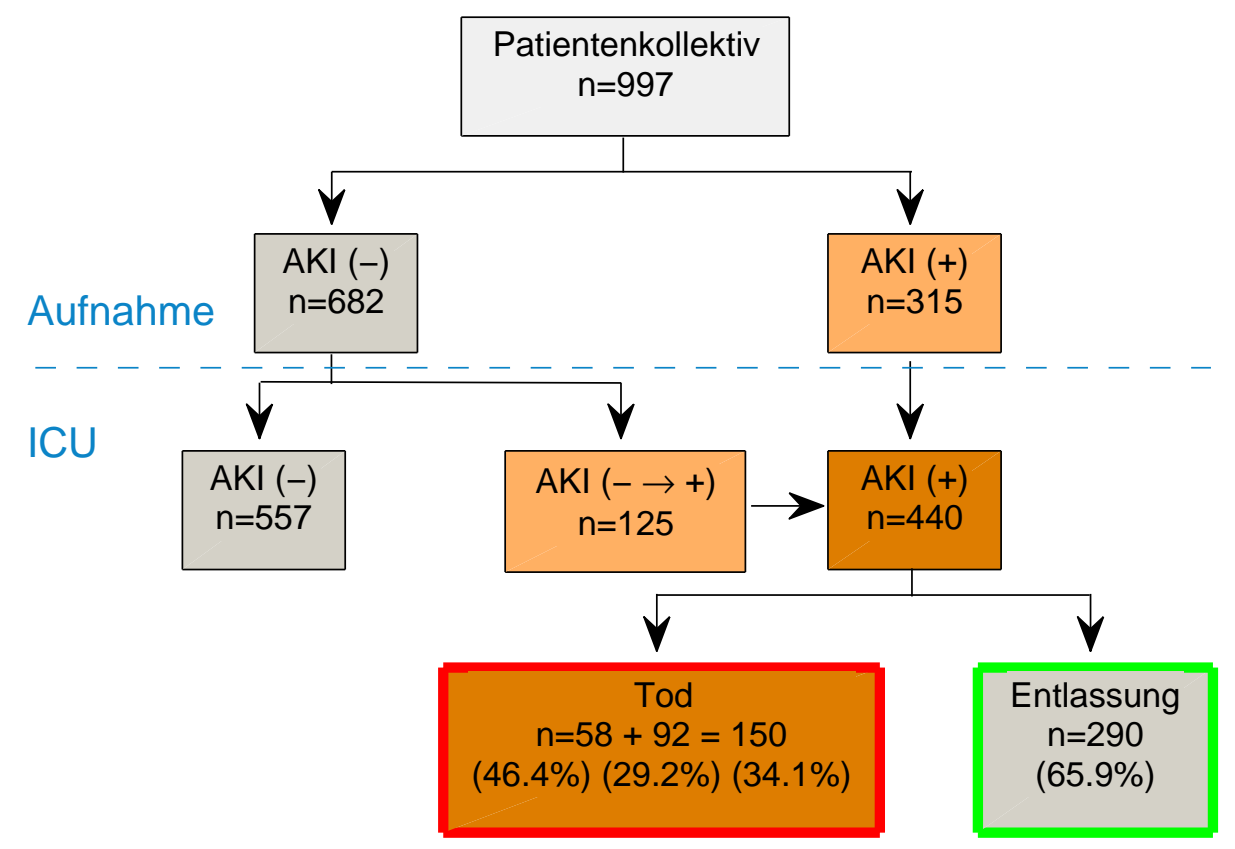

AbBiLdung 3.9: Prävalenz und Mortalität des akuten Nierenversagens im Patientenkollektiv.

Nierenerkrankungen). Nur bei 3,0\% der Patienten lagen postrenale Ursachen (Harnabflußstörungen unterschiedlicher Genese) vor; bei 4,8\% der Fälle ließ sich eine Ursache des AKI nicht ermitteln.

Anhand der Patientendokumentation wurde die Frage des Einflusses potentiell nephrotoxischer Substanzen auf die Entwicklung eines AKI untersucht. In Ermangelung der Feststellung anderer Ursachen bestand bei 28 von insgesamt 440 AKI-Patienten der dringende Verdacht eines medikamentös-induzierten nephrotoxischen AKI (Chemotherapeutika 78,6\%; nicht-steroidale Antirheumatika 10,7\%; Statine 10,7\%).

Von den Patienten, die - soweit in ihrer Vorgeschichte nachweisbar - sowohl als Vormedikation bzw. während der Behandlung auf der ICU keine potentiell nephrotoxischen Medikamente erhalten hatten, entwickelten 22, $2 \%$ ein AKI. Bei bekannter Einnahme von 1 - 4 oder mehr nephrotoxischen Medikamenten nahm die Prävalenz der AKI mit Anzahl der Medikamente zu: 35,8\%<42,9\% $242,7 \%<48,0 \%(p=0,024)$. Bei Betrachtung der Wirkstoffgruppen war die Einnahme von Protonenpumpeninhibitoren $(43,2 \%$ vs. $32,0 \%, p<0,031)$ und von Allopurinol $(16,5 \%$ vs. $4,6 \%, p<0,001)$ mit der Entwicklung eines AKI assoziiert. 
Die Klassifizierung des AKI nach Schweregraden wurde in Kap. 2.5.1 bereits dargestellt. Gemäß der AKIN-Klassifizierung lag bei 18, 1\% der Patienten ein Stadium I vor, 9, 4\% befanden sich im Stadium II, 72, 5\% im Stadium III. Bei 269 Patienten lag keine Klassifikation entsprechend der AKIN-Kriterien vor. Insgesamt lag bei 202 (45,9\%) Patienten ein AKIN-Stadium III vor. Bei der Klassifizierung der AKIN-Stadien I - III muss beachtet werden, dass es sich nicht um eine statische Klassifizierung handelt, vielmehr werden überwiegend im Verlauf der Entwicklung eines AKI innerhalb eines kurzen Zeitraums die Stadien I - III durchlaufen. Patienten mit AKIN-Stadium III hatten bereits vergleichsweise höhere $\left[K r_{s}\right]$-Ausgangswerte $(1,5 \pm 0,79 \mathrm{mg} / \mathrm{dl})$ als Patienten in den AKIN-Stadien I - II $(0,79 \mathrm{mg} / \mathrm{dl})$. Ein anurischer Verlauf des AKI bestand bei 10,9\% der Patienten.

Die Merkmale zur Beurteilung des Behandlungserfolgs und die Risikofaktoren sind in Tab. 3.3 zusammengefaßt.

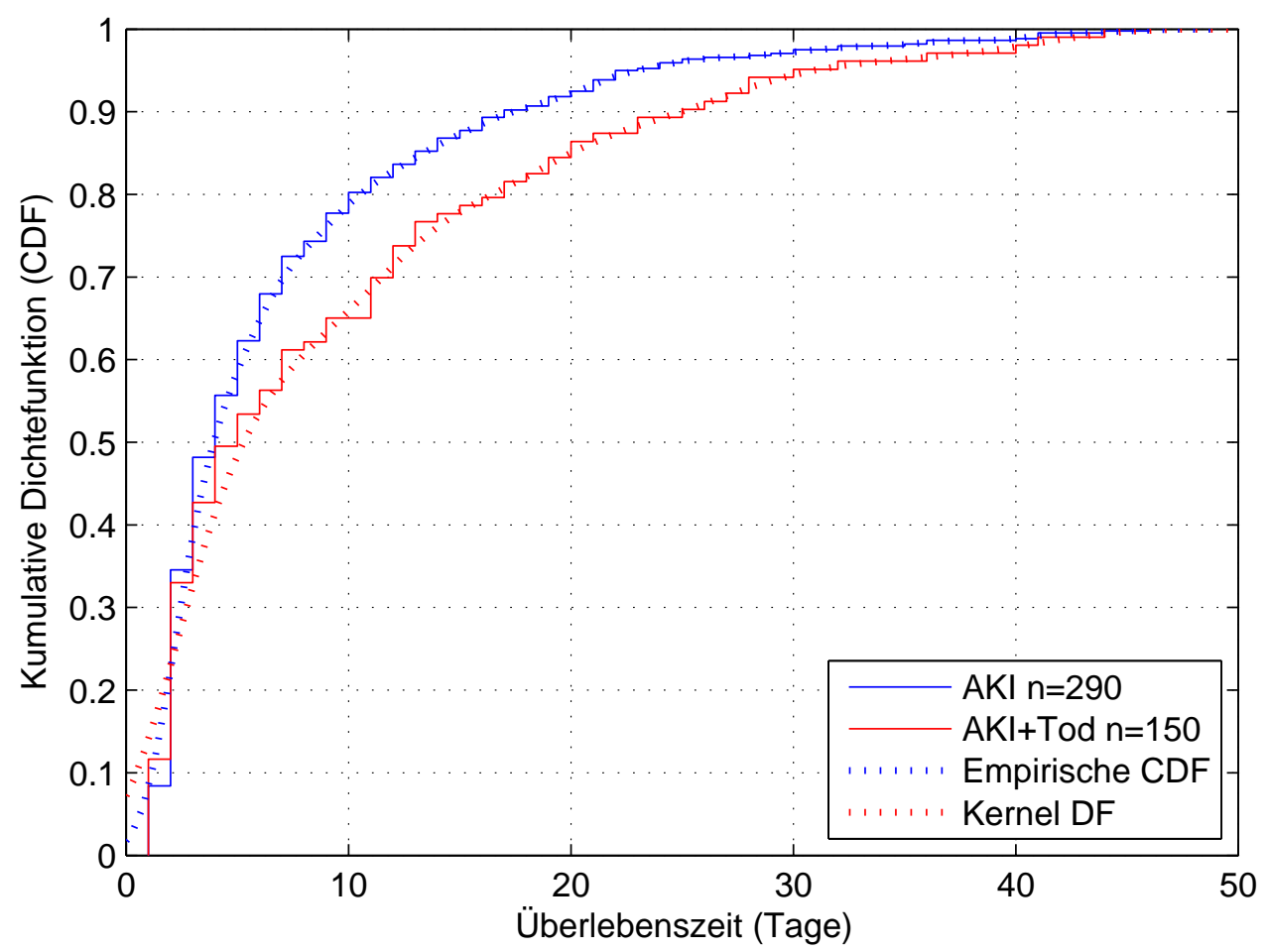

ABBILDung 3.10: Mortalität des akuten Nierenversagens im Patientenkollektiv.

Die Mortalität bei den Patienten, die bereits mit einem vorbestehenden AKI zur Aufnahme auf die ICU kamen, betrug 29, 2\%. Patienten, die erst auf der ICU ein AKI entwickelten, hatten eine Mortalität von 46,4\% $(p<0,001)$. Die Inzidenz der Entwicklung eines AKI während der Behandlung auf der ICU führte zu einem Anstieg der Mortalität um 16,7\%. Die Gesamtmortalität aller Patienten mit AKI betrug 34,1\%. Nach den 


\begin{tabular}{|c|c|c|c|c|c|c|c|c|c|c|c|c|c|c|c|}
\hline 2 & $\begin{array}{l}\vec{N} \\
\tilde{0}\end{array}$ & $\begin{array}{l}\overrightarrow{8} \\
0 \\
0 \\
v\end{array}$ & $\begin{array}{l}\overrightarrow{8} \\
0 \\
0 \\
V\end{array}$ & $\begin{array}{l}\overrightarrow{8} \\
0 \\
0 \\
v\end{array}$ & $\vec{\sigma}$ & $\begin{array}{l}\overrightarrow{8} \\
0 \\
0 \\
1\end{array}$ & $\begin{array}{l}2 \\
0 \\
0 \\
V\end{array}$ & $\begin{array}{l}\mathscr{\sigma} \\
0 \\
0\end{array}$ & $\begin{array}{l}7 \\
0 \\
0\end{array}$ & $\begin{array}{l}8 \\
10 \\
0\end{array}$ & $\begin{array}{l}\overrightarrow{8} \\
0 \\
0 \\
1\end{array}$ & $\begin{array}{l}\overrightarrow{8} \\
0 \\
0 \\
v\end{array}$ & $\begin{array}{l}\sigma \\
0 \\
v\end{array}$ & $\begin{array}{l}\overrightarrow{8} \\
0 \\
0 \\
v\end{array}$ & $\begin{array}{l}\overrightarrow{8} \\
0 \\
0 \\
1\end{array}$ \\
\hline $\begin{array}{l}\approx \\
\approx \\
0 \\
0 \\
0 \\
0\end{array}$ & 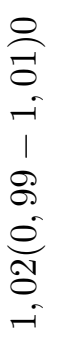 & 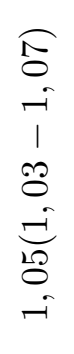 & 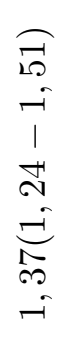 & 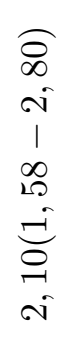 & & $\begin{array}{c}\overparen{\infty} \\
\rightarrow \\
0 \\
1 \\
1 \\
20 \\
0 \\
0 \\
0 \\
0 \\
-1\end{array}$ & 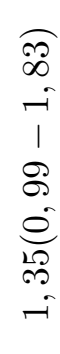 & $\begin{array}{l}\widehat{0} \\
-1 \\
1 \\
0 \\
0 \\
e \\
0 \\
0 \\
0 \\
-1\end{array}$ & 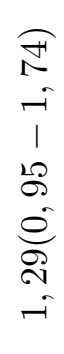 & 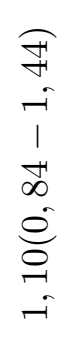 & 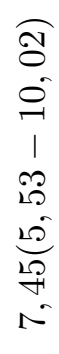 & 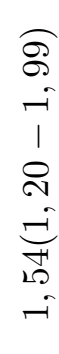 & 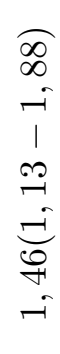 & 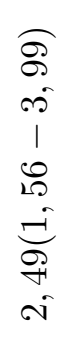 & 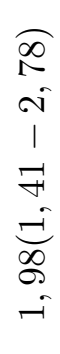 \\
\hline 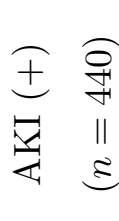 & $\begin{array}{l}0 \\
\vec{H} \\
\vec{H} \\
H \\
\vec{H} \\
0\end{array}$ & $\begin{array}{l}\overrightarrow{1} \\
\dot{\theta} \\
+1 \\
0 \\
\dot{\theta}\end{array}$ & $\begin{array}{l}\infty \\
-1 \\
= \\
H \\
\infty \\
\infty \\
\infty\end{array}$ & 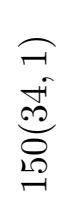 & 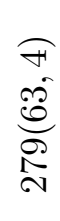 & 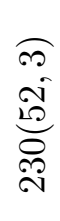 & $\underset{\substack{\infty \\
\stackrel{\infty}{0}}}{\stackrel{0}{0}}$ & 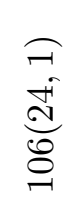 & 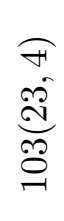 & 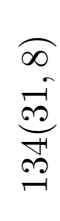 & $\begin{array}{l}0 \\
0 \\
0 \\
10 \\
20 \\
20 \\
\text { N }\end{array}$ & 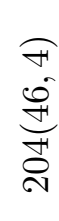 & $\begin{array}{l}\overparen{N} \\
\stackrel{\infty}{\infty} \\
\underset{N}{+} \\
\stackrel{N}{N}\end{array}$ & 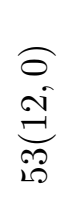 & $\frac{\sqrt[0]{20}}{\sqrt[20]{21}}$ \\
\hline 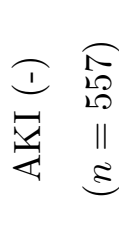 & $\begin{array}{l}0 \\
0 \\
= \\
H \\
1 \\
10 \\
0\end{array}$ & $\begin{array}{l}0 \\
0 \\
+1 \\
-1 \\
0\end{array}$ & $\begin{array}{l}\vec{H} \\
\vec{H} \\
\vec{H} \\
\vec{H}\end{array}$ & 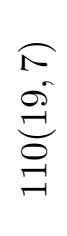 & $\begin{array}{l}60 \\
60 \\
10 \\
10 \\
-0\end{array}$ & $\begin{array}{l}0 \\
0 \\
\stackrel{0}{\rightleftharpoons} \\
0 \\
0 \\
-1\end{array}$ & $\begin{array}{l}\widehat{\sigma} \\
\infty \\
\stackrel{\infty}{=} \\
\stackrel{20}{0}\end{array}$ & 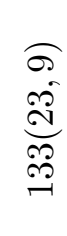 & $\begin{array}{l}\overparen{N} \\
\stackrel{\sigma}{\Xi} \\
\stackrel{5}{0}\end{array}$ & $\begin{array}{l}0 \\
0 \\
\stackrel{0}{0} \\
0 \\
0\end{array}$ & $\begin{array}{l}\underset{\infty}{6} \\
\underset{\infty}{10} \\
\rightleftarrows\end{array}$ & 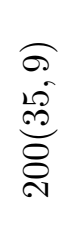 & 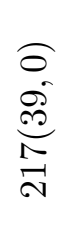 & $\begin{array}{l}\overparen{\sim} \\
\underset{\mathscr{D}}{\stackrel{D}{N}}\end{array}$ & $\begin{array}{l}\overparen{N} \\
\stackrel{\sim}{ \pm} \\
\stackrel{D}{0}\end{array}$ \\
\hline 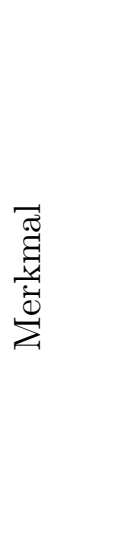 & 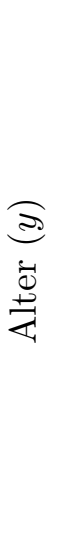 & 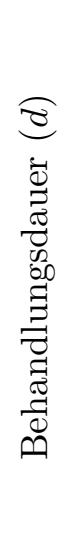 & 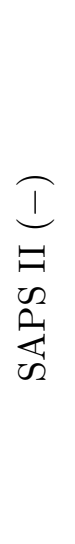 & 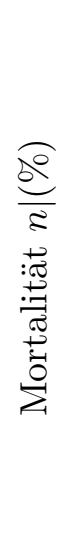 & 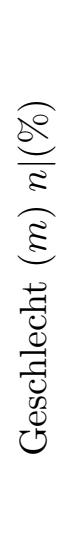 & 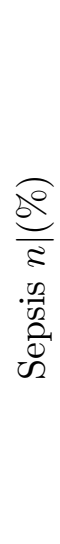 & 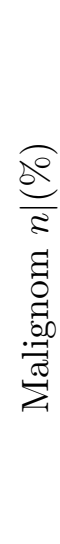 & 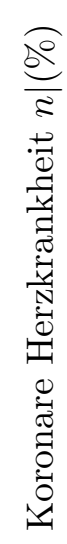 & 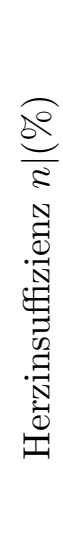 & 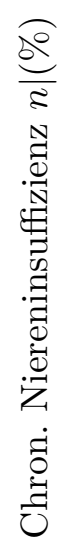 & 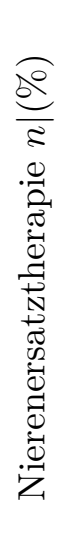 & 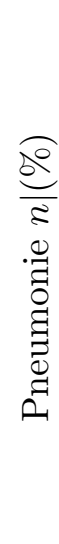 & 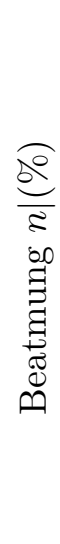 & 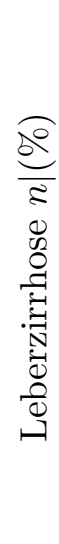 & 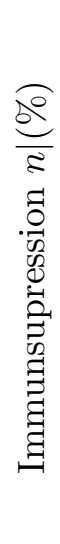 \\
\hline
\end{tabular}


AKIN-Stadien I - III geordnet, betrug die Mortalität mit den Stadien ansteigend 22, 6\%, $31,3 \%$ und 35, 5\%. Mit anurischem Nierenversagen verstarben 43, $8 \%$ der Patienten.

Die kumulativen Dichtefunktionen (s. Erläuterungen im Text zu Abb. 3.4) der AKIPatienten mit positivem Behandlungserfolg (Überleben und Entlassung von der ICU) und mit negativem Behandlungserfolg (Tod auf der ICU) sind in Abb. 3.10 dargestellt. Für die Verstorbenen ist die Behandlungsdauer auf der ICU gleichbedeutend mit der Überlebenszeit. Die Wahrscheinlichkeitswerte (probability, $P=0.75$ ) der Überlebenden (8,4 Tage) und der Verstorbenen (13,5 Tage) bedeuten, dass die AKI-Verstorbenen eine längere Behandlungszeit auf der ICU erfuhren, auch wenn diese im Ergebnis den Todeseintritt nicht abwenden konnte.

Die klinischen Laborwerte der Patienten, die bereits mit einem vorbestehenden AKI [AKI $(+)$ ] zur Aufnahme auf die ICU kamen $(n=315)$, sind in Tab. 3.4 zusammengestellt. Diese hatten bereits um den Faktor $\sim 2$ erhöhte Werte der harnpflichtigen Substanzen. Auffällig war die in beiden Gruppen verminderte Hämoglobin-Konzentration.

Innerhalb des Gesamtkollektivs $(n=997)$ bestand bei $682(68,4 \%)$ zum Zeitpunkt der ICU-Aufnahme nach Laborwerten und sonstigen klinischen Untersuchungsergebnissen kein Anfangsverdacht auf das Vorliegen einer Nierenfunktionsstörung. Ungeachtet des Fehlens jeglicher Anhaltspunkte für ein imminentes AKI, entwickelten 125 (12,5\%) Patienten ein AKI während der Behandlungsdauer auf der ICU, dies aus Gründen der akuten Exazerbation oder Dekompensation einer andersartigen Grundkrankheit. Die statischen Zahlenwerte aus Tab. 3.4 bei der ICU-Aufnahme, inbesondere die für $\left[K r_{s}\right]$ und Harnstoff, stellen keine Frühindikatoren für die imminente Gefahr der Entwicklung eines AKI dar. Entscheidend für die Diagnose eines AKI ist allein die Dynamik, d.h. die zeitliche Entwicklung der Veränderung dieser Laborparameter, wie sie in den AKINKriterien des AKI festgelegt wurde (s. Tab. 2.2). Die kumulierten Histogramme der $\left[K r_{s}\right]$-Konzentrationen zeigten eine erkennbare Verringerung der $\left[K r_{s}\right]$-Konzentrationen zum Zeitpunkt der Entlassung, allerdings bei Weitem keine Restitution auf Normalwerte.

\subsection{Akutes Nierenversagen und Sepsis}

Die Prävalenz der Sepsis ( $n=336 ; m 212$ (63, 1\%), $w 124$ (36, 9\%)) im Patientenkollektiv lag bei 33,7\%. Eine Übersicht der Patienten mit Sepsis gibt die Abb. 3.12.

Ein mikrobiologischer Keimnachweis gelang in 25,3\% der Fälle. Die Übersicht der Erregerverteilung ist in Abb. 3.13 zusammengefaßt. Erreger aus der Gruppe der Enterobacteriaceae waren mit $33 \%$ am häufigsten vertreten, hierunter war Escherichia coli der 


\begin{tabular}{|c||c|c|c|}
\hline \hline Variable bei ICU-Aufnahme & $\begin{array}{c}\text { AKI }(-) \\
(n=682)\end{array}$ & $\begin{array}{c}\text { AKI }(+) \\
(n=315)\end{array}$ & $p$ \\
\hline Kreatinin $(m g / d l)$ & $0,89(0,49)$ & $2,78(2,88)$ & $<0,001$ \\
Harnstoff $(m g / d l)$ & $26(23,0)$ & $59(42,5)$ & $<0,001$ \\
Kalium $(\mathrm{mmol} / \mathrm{l})$ & $4,2(0,8)$ & $4,4(1,2)$ & 0,81 \\
Natrium $(\mathrm{mmol} / \mathrm{l})$ & $138(7)$ & $138(8)$ & 0,37 \\
Hämoglobin $(\mathrm{g} / \mathrm{dl})$ & $10,9(3,8)$ & $10,1(2,8)$ & 0,90 \\
Thrombozyten $\left(10^{3} / \mu l\right)$ & $217(147)$ & $158(147)$ & 0,02 \\
\hline
\end{tabular}

TABelLe 3.4: Laborwerte der AKI Patienten bei ICU-Aufnahme. Median (Interquartilsabstand).
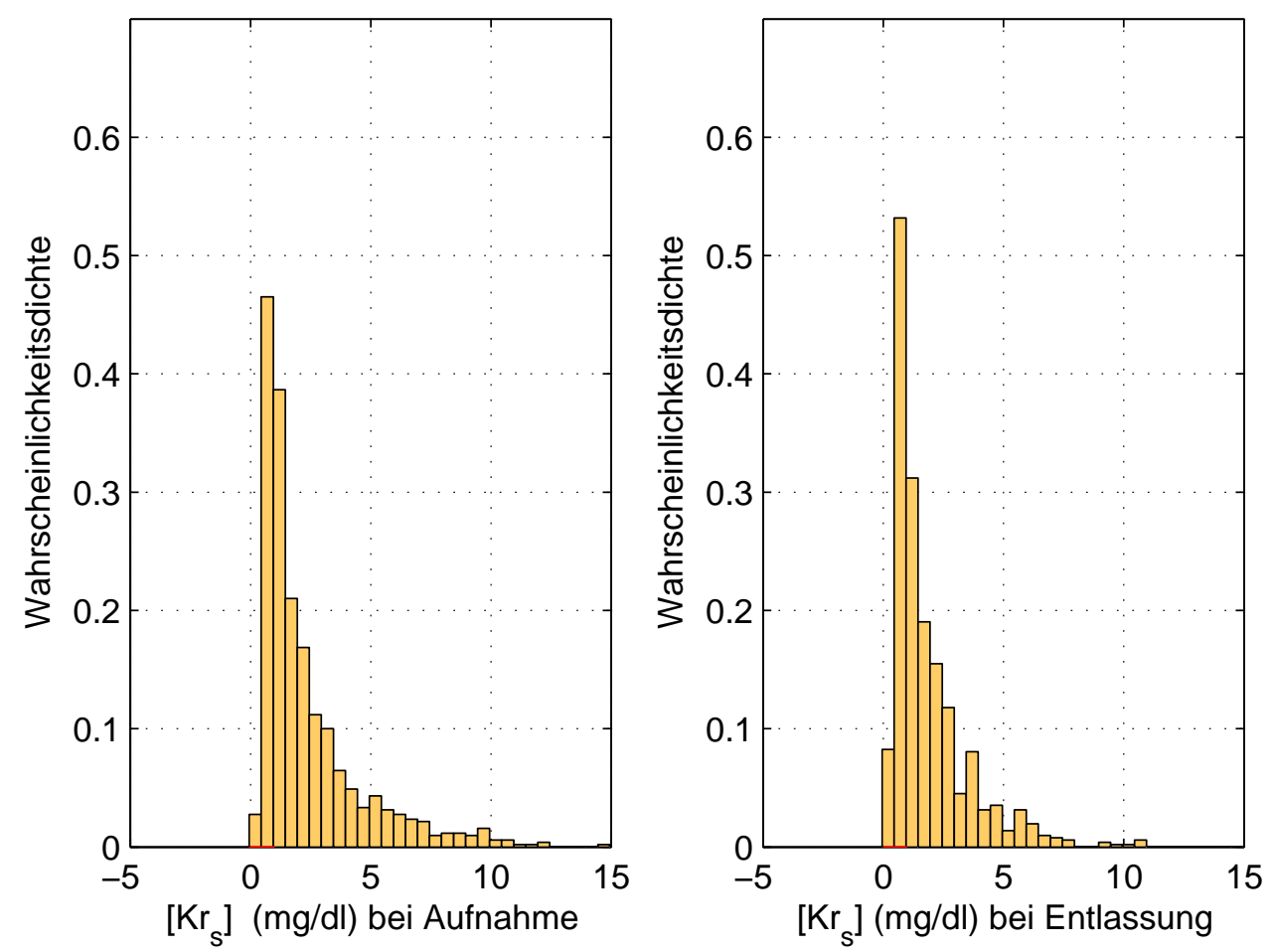

ABBILDUng 3.11: Kreatinin-Konzentration bei Aufnahme und Entlassung. 


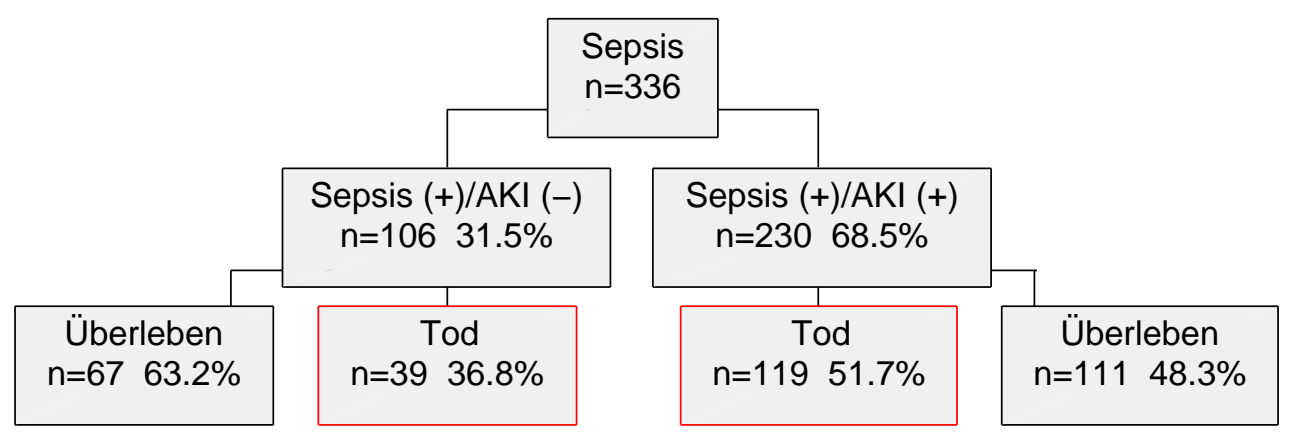

AbBILDUng 3.12: Prävalenz von AKI und Sepsis.

häufigste opportunistische Erreger. Die zweithäufigste Gruppe waren Staphylococcaceae (Staphylococcus aureaus, Staphylococcus epidermidis) mit $26 \%$.

Nach den Kriterien der Internationalen Sepsis-Konferenz (s. Kap. 2.5.2) lag bei den Sepsis-Patienten die folgende differenzierende Verteilung der Verlaufsformen vor: SIRS 7,4\%, Sepsis 44,0\%, schwere Sepsis 16, 4\%, septischer Schock 32, $1 \%$.

Die Prävalenz und Mortalität der Sepsis-Patienten ist in Abb. 3.14 zusammengefaßt. Es wurden die Patientengruppen $[\operatorname{Sepsis}(+\mid-) / \operatorname{AKI}(+\mid-)]$ und $[\operatorname{Sepsis}(+) / \operatorname{AKI}(+)]$ vergleichend betrachtet. In der Gruppe der Patienten, die keine Sepsis entwickelten, [Sepsis (-)/AKI(+)], lag die Prävalenz des AKI bei 31,5\%. Dagegen war die Prävalenz des AKI bei Patienten mit Sepsis, [Sepsis $(+) / \operatorname{AKI}(+)]$, mit 68,5\% mehr als 2-fach erhöht.

Mehr als 2/3 der Patienten [Sepsis(+)/AKI(+)] vs. [Sepsis(+)/AKI(-)], (68,5\% vs. $31,5 \%)$ entwickelten ein AKI mit tödlichem Ausgang in mehr als der Hälfte der Fälle (51,7\% vs. 36, 8\%, p<0,001). Bei Patienten ohne Sepsis [Sepsis(-)/AKI(-)] vs. [Sepsis $(-) / \operatorname{AKI}(+)]$ war die Mortalität deutlich geringer $(16,0 \%$ vs. $14,3 \%, p=0,58)$.

Die Mortalität in den nach Schweregraden stratifizierten Untergruppen war mit dem Schweregrad des septischen Verlaufs ansteigend: SIRS 12\%, Sepsis 40\%, schwere Sepsis $55 \%$, septischer Schock $62 \%$.

Die häufigste Begleiterkrankung der Sepsis war die Pneumonie (60,4\% vs. 30,4\%). Eine Nierenersatztherapie war 2-fach häufiger erforderlich als bei Patienten ohne Sepsis (50,3\% vs. $26,2 \%)$.

In Abb. 3.15 ist die Prävalenz der verschiedenen AKIN-Stadien (s. Kap. 2.5.2) bei den verschiedenen Patientengruppen, [Sepsis $(+\mid-) /$ AKIN I-III], dargestellt. In den Gruppen [Sepsis(-)/AKIN I] vs. [Sepsis (+)/ AKIN I] war das AKIN-Stadium I signifikant häufiger 


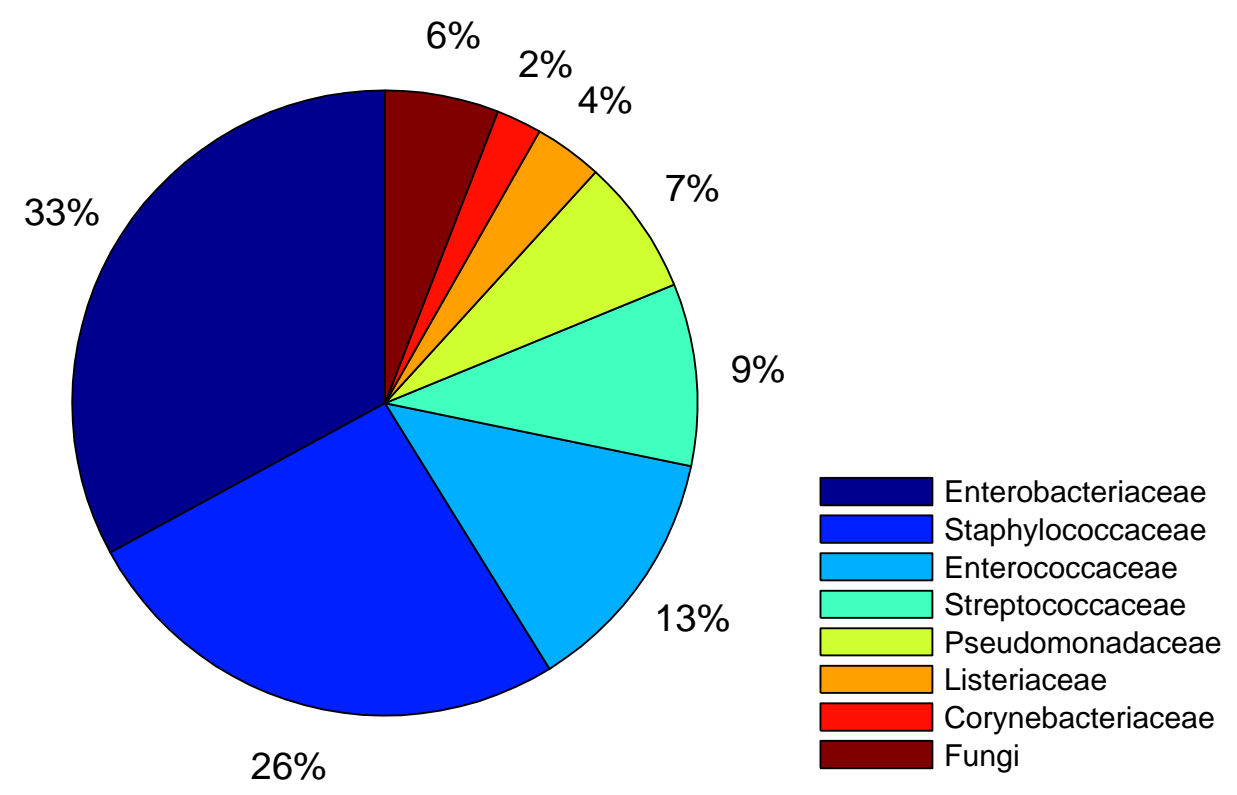

AbBILDung 3.13: Verteilung bakterieller Erreger bei Sepsis.

als bei Patienten ohne Sepsis $(27,8 \%$ vs. $11,5 \%, p=0,01)$. Dagegen war bei Patienten mit Sepsis [Sepsis(-)/AKIN III] vs. [Sepsis (+)/AKIN III] das AKIN-Stadium III signifikant häufiger vertreten $(65,3 \%$ vs. $77,1 \%, p=0,01)$.

Die Betrachtung der Mortalität nach den AKIN-Stadien des AKI bei Sepsis ergibt jedoch, dass bei unterschiedlicher Prävalenz der AKIN-Stadien I - III keine signifikanten Unterschiede in der Mortalität bestehen (AKIN I/II 59,1\%, AKIN III 48,6\%, $p=0,47$ ). Einschränkend muss aber darauf hingewiesen werden, dass nur in 19,3\% der AKIPatienten eine eindeutige Klassifizierung nach den AKIN-Kriterien vorlag.

Die Merkmale zur Beurteilung des Behandlungserfolgs und die Risikofaktoren sind in Tab. 3.5 zusammengefaßt.

Zusammenfassend ergibt die Analyse folgende wesentliche Feststellungen:

(i) Die Mortalität bei der septischen Erkrankung steigt annährend proportional mit dem Schweregrad von $12 \%-62 \%$. 


\begin{tabular}{|c|c|c|c|c|c|c|c|c|c|c|c|c|c|c|}
\hline 2 & $\begin{array}{l}8 \\
0 \\
0\end{array}$ & $\begin{array}{l}\vec{\sigma} \\
0 \\
0 \\
v\end{array}$ & $\begin{array}{l}\vec{\sigma} \\
0 \\
\dot{0} \\
v\end{array}$ & $\begin{array}{l}\overrightarrow{8} \\
0 \\
0 \\
1\end{array}$ & $\begin{array}{l}\overrightarrow{8} \\
0 \\
0 \\
v\end{array}$ & $\begin{array}{l}5 \\
8 \\
0 \\
1\end{array}$ & $\begin{array}{l}0 \\
0 \\
0\end{array}$ & $\hat{\sigma}$ & $\begin{array}{l}\stackrel{P}{+} \\
0\end{array}$ & $\begin{array}{l}\overrightarrow{8} \\
0 \\
0 \\
V\end{array}$ & $\begin{array}{l}\tilde{\delta} \\
0 \\
v\end{array}$ & $\begin{array}{l}\overrightarrow{8} \\
0 \\
0 \\
v\end{array}$ & $\begin{array}{l}\tilde{I} \\
\tilde{O} \\
0 \\
V\end{array}$ & $\begin{array}{l}\tilde{8} \\
0 \\
0 \\
V\end{array}$ \\
\hline$\therefore \begin{array}{l}1 \\
0 \\
0 \\
0 \\
0\end{array}$ & $\begin{array}{l}\widehat{\sigma} \\
-i \\
1 \\
8 \\
- \\
- \\
0 \\
-i\end{array}$ & 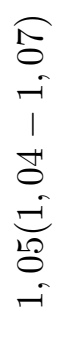 & 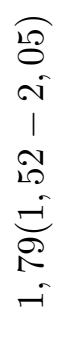 & 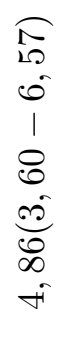 & 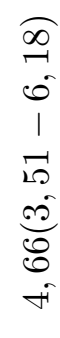 & 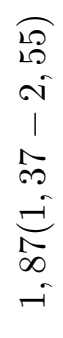 & 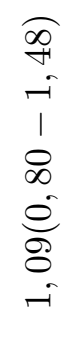 & 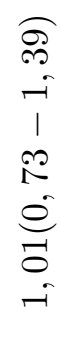 & 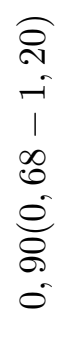 & 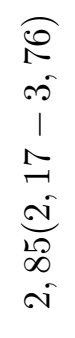 & 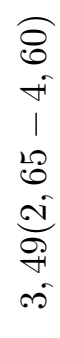 & 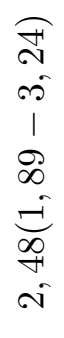 & 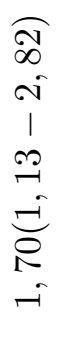 & $\begin{array}{c}0 \\
8 \\
\infty \\
1 \\
20 \\
20 \\
- \\
\infty \\
-1 \\
-1 \\
-1\end{array}$ \\
\hline 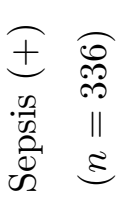 & $\begin{array}{l}0 \\
0 \\
\stackrel{9}{7} \\
H \\
\overrightarrow{0}\end{array}$ & 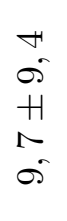 & $\begin{array}{l}\infty \\
\stackrel{\infty}{*} \\
\sim \\
+ \\
\infty \\
\infty \\
\infty\end{array}$ & 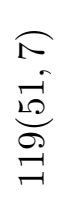 & 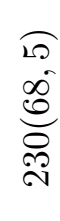 & $\begin{array}{l}\overparen{N} \\
\stackrel{0}{0} \\
\stackrel{d}{2} \\
0\end{array}$ & 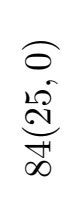 & $\begin{array}{l}\overparen{F} \\
\stackrel{\vec{N}}{E}\end{array}$ & $\begin{array}{l}\text { 20 } \\
2 \\
\stackrel{2}{2}\end{array}$ & 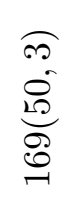 & $\begin{array}{l}\text { F } \\
\dot{8} \\
\dot{0} \\
\tilde{\theta} \\
\tilde{\theta}\end{array}$ & 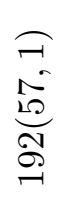 & 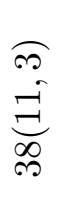 & $\begin{array}{c}\overparen{\infty} \\
\stackrel{\infty}{\stackrel{\infty}{\infty}} \\
\infty\end{array}$ \\
\hline 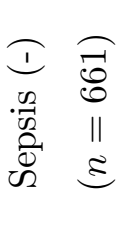 & $\begin{array}{l}\infty \\
0 \\
-1 \\
+1 \\
-1 \\
0\end{array}$ & $\begin{array}{l}N \\
N \\
H \\
N \\
0\end{array}$ & $\begin{array}{l}\infty \\
0 \\
-1 \\
H \\
20 \\
0 \\
0\end{array}$ & 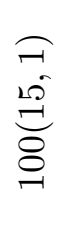 & 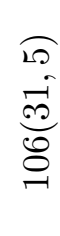 & $\begin{array}{l}\stackrel{\overbrace{}}{F} \\
\stackrel{-}{=} \\
\stackrel{20}{=} \\
=\end{array}$ & 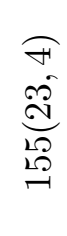 & 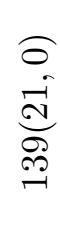 & 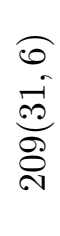 & 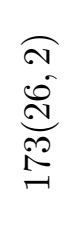 & $\begin{array}{l}\text { F } \\
\stackrel{0}{8} \\
\stackrel{8}{=} \\
\stackrel{\circ}{\circ}\end{array}$ & 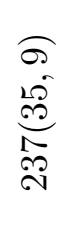 & $\begin{array}{l}\overparen{E} \\
\stackrel{0}{\mathscr{H}} \\
\dot{H}\end{array}$ & $\begin{array}{c}\overparen{6} \\
\stackrel{1}{\Xi} \\
\stackrel{\Xi}{\infty}\end{array}$ \\
\hline 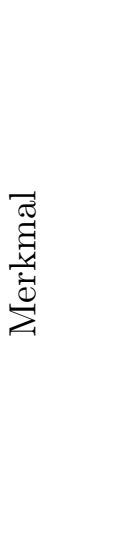 & 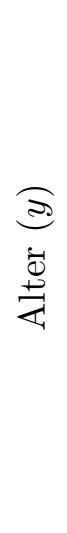 & 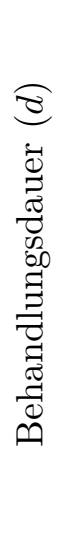 & 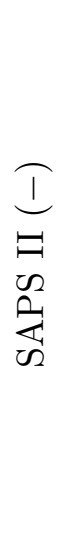 & 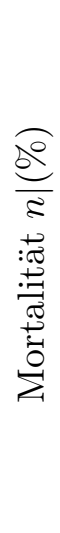 & $\begin{array}{l}\frac{\pi}{2} \\
\frac{2}{4}\end{array}$ & 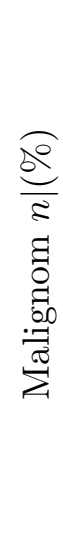 & 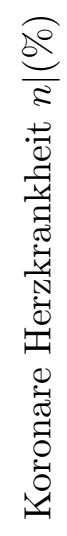 & 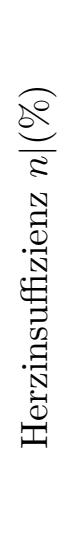 & 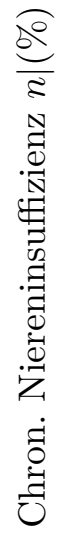 & 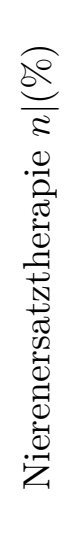 & 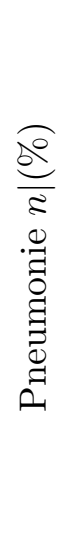 & 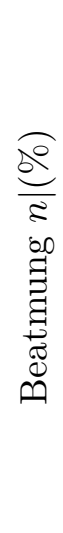 & 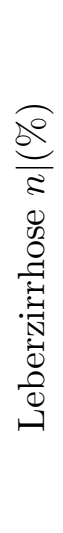 & 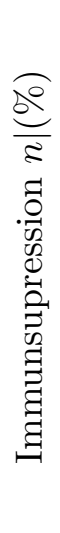 \\
\hline
\end{tabular}



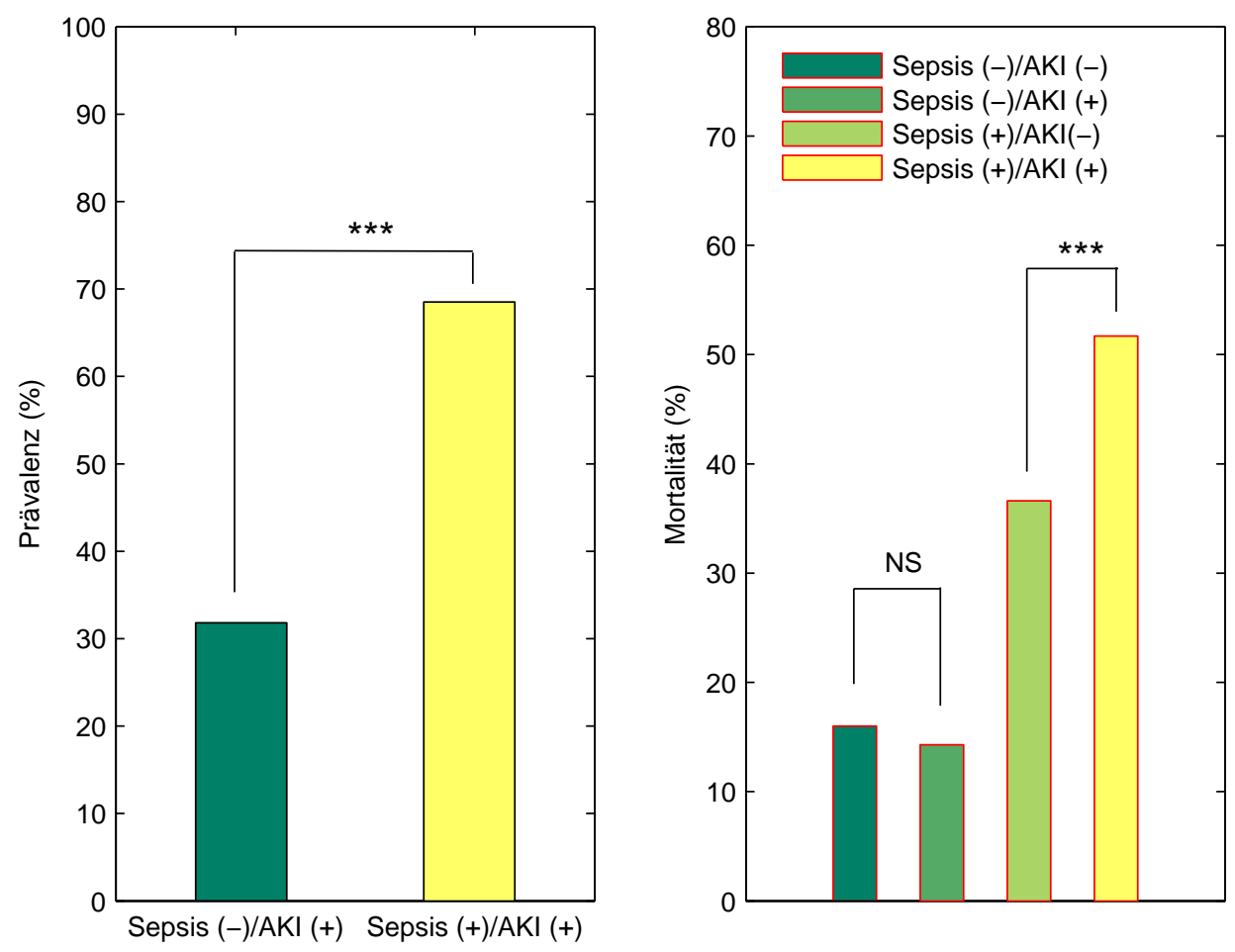

AbBILDung 3.14: Prävalenz und Mortalität von Sepsis und AKI.

(ii) Die Koinzidenz des AKI bei septischer Grunderkrankung ist mit 68, $5 \%$ außerordentlich hoch und betrifft mehr als 2/3 der Patienten.

(iii) Die Mortalität bei Koinzidenz von AKI und Sepsis ist mit 51,7\% ebenfalls außerordentlich hoch und betrifft mehr als $1 / 2$ der Patienten.

(iv) Bei der Koinzidenz von AKI und Sepsis besteht kein eindeutiger Zusammenhang zwischen der Dynamik bzw. dem Schweregrad der Entwicklung des AKI (nach den AKINKriterien) und der Mortalität.

\subsection{Malignom}

Die Prävalenz maligner Tumorerkrankungen $(n=210 ; m 138$ (65,7\%), w 72 (34,3\%)) im Patientenkollektiv lag bei 21,1\%. Eine Übersicht der Patienten mit Malignom gibt die Abb. 3.16. 


\begin{tabular}{|c|c|}
\hline Sepsis (-) \& AKIN & Sepsis (+) \& AKIN \\
\hline Sepsis (-)/AKIN & Sepsis (+)/AKIN I \\
\hline $20 / 72 \quad 27.8 \%$ & $11 / 96 \quad 11.5 \%$ \\
\hline Sepsis (-) / AKKIN \| & Sepsis (+) / AKIN \| \\
\hline $6.9 \%$ & $11.96 \quad 11.5 \%$ \\
\hline Sepsis (-) / AKIIN III & Sepsis (+) / AKIN III \\
\hline $47 \pi 2 \quad 65.3 \%$ & $74 / 96 \quad 77.1 \%$ \\
\hline
\end{tabular}

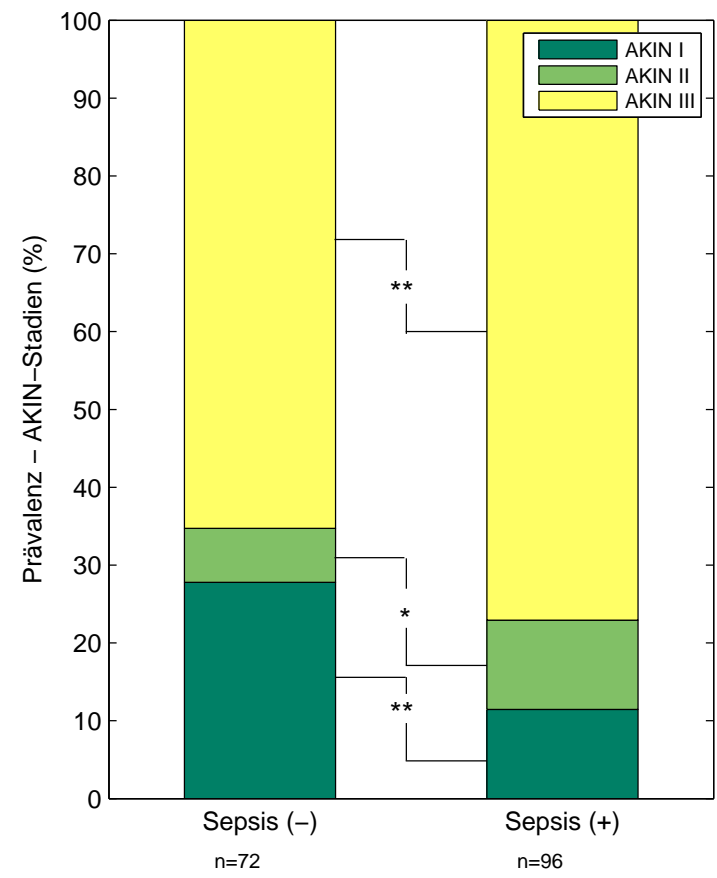

AbBiLdung 3.15: Prävalenz der AKIN-Stadien und Sepsis.

Bei den malignen Neoplasien wurde eine Unterscheidung nach soliden Tumoren und Hämoblastosen vorgenommen. Solide Tumoren bestanden bei 58,6\% der Patienten, Hämoblastosen bei 41,4\%. Fernmetastasen solider Tumoren lagen in 13,8\% der Fälle vor. Die Verteilung maligner Neoplasien im Patientenkollektiv ist in Abb. 3.17 dargestellt. Die weitere Aufteilung der Hämoblastosen ist im Einzelnen in Abb. 3.18 angegeben.

Unter den malignen Tumorerkrankungen waren Hämoblastosen mit $41 \%$ der dominierende Anteil. Bei den hämato-onkologischen Erkrankungen war die akute myeloische Leukämie mit 26\% die häufigste Form. Mehr als 1/3 der Patienten mit Hämoblastosen $(37,9 \%)$ hatten in der Vergangenheit eine Stammzell-Transplantation erhalten.

Die Merkmale zur Beurteilung des Behandlungserfolgs und die Risikofaktoren sind in Tab. 3.6 zusammengefaßt. Aus den ausgewiesenen Quotenverhältnissen ( $R$, Erläuterungen s. Kap. 3.5) ergeben sich die Merkmale und Begleitumstände, die Anlass zu der Vermutung eines wahrscheinlichen bzw. erhöhten Risikos des Todeseintritts geben. Hierbei stellt die assoziierte Sepsis $(R=6,8)$ eine bedeutende Komplikation dar, ebenso wie die Koinzidenz eines AKI $(R=4,0)$. Die Notwendigkeit der künstlichen Beatmung ist ebenfalls mit einem erhöhten Mortalitätsrisiko behaftet $(R=4,8)$. Der Schweregrad der Erkrankung nach den SAPS II-Kriterien (s. Kap. 2.8) war bei den Verstorbenen signifikant erhöht. 


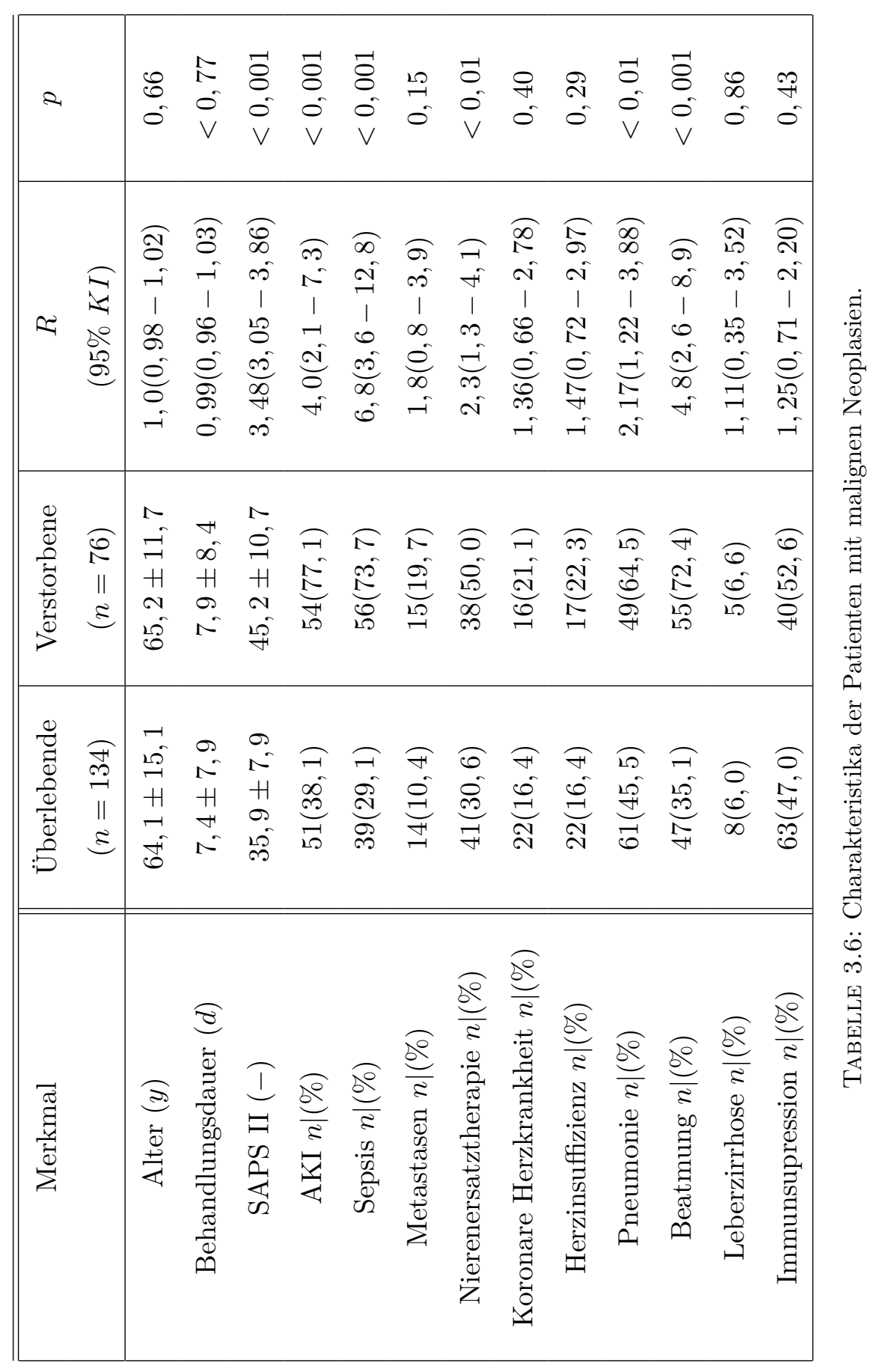




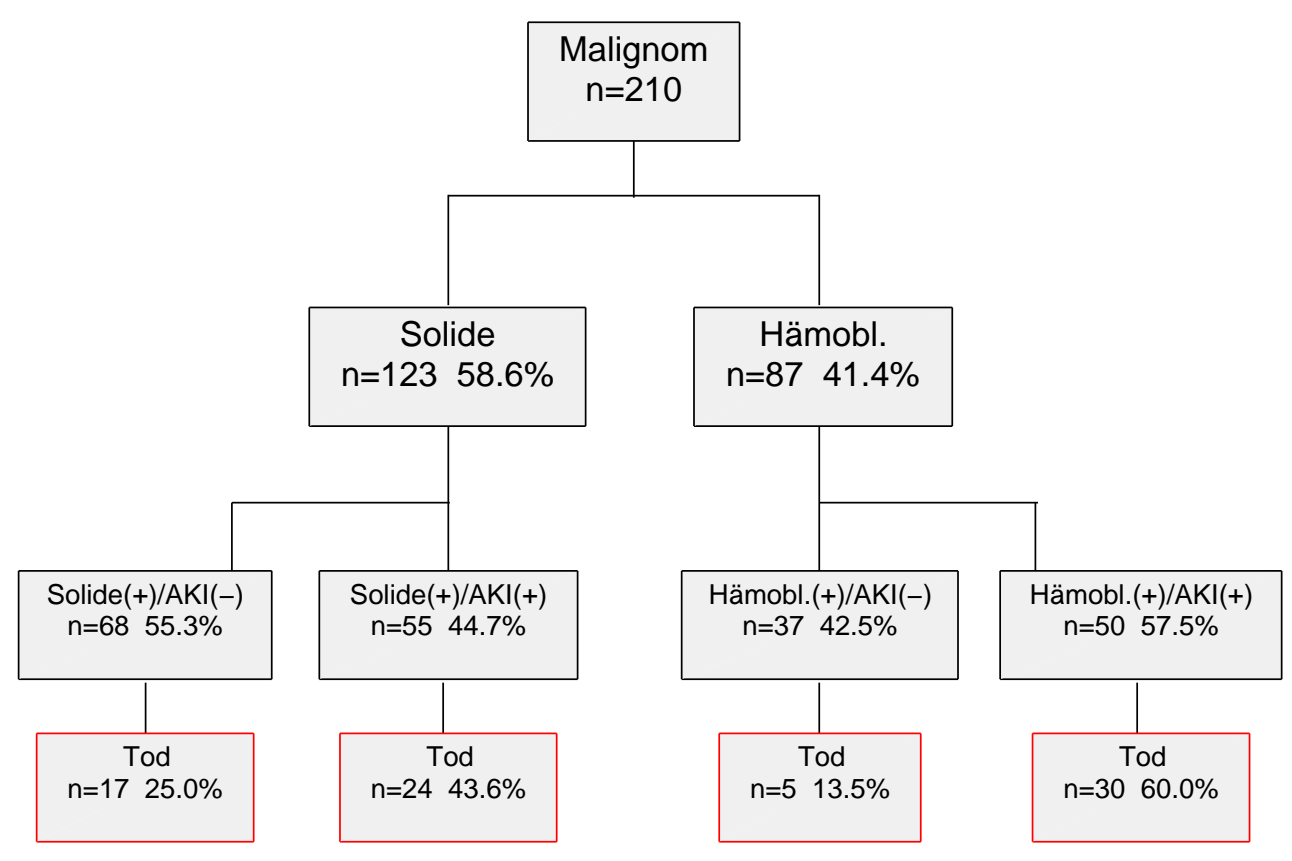

AbBiLdung 3.16: Prävalenz und Mortalität maligner Neoplasien.

Die Charakteristika der Patienten mit malignen Neoplasien, aufgeteilt nach dem Vorliegen solider Tumoren und Hämoblastosen, sind in Tab. 3.7 zusammengestellt. Die Patienten mit Hämoblastosen waren deutlich jünger (68,8 vs. 58,1 y). Die Prävalenz einer Sepsis war bei Patienten mit Hämoblastosen gegenüber denen mit soliden Tumorerkrankungen deutlich erhöht $(56,3 \%$ vs. $37,4 \%)$. Es bestand eine signifikante Prävalenz für die Entwicklung eines AKI.

\subsection{Akutes Nierenversagen und maligne Neoplasien}

Die Prävalenz des AKI bei Patienten mit Malignom ist in Abb. 3.19 (links) dargestellt. Der Vergleich der Untergruppen der Patienten ohne Malignom [Malignom (-)/AKI $(+)$ ] mit denen, die an einem Malignom erkrankt waren (ohne Unterscheidung zwischen soliden und hämoblastischen Neoplasien) [Malignom $(+) /$ AKI $(+)$ ] ergab eine höhere Prävalenz des AKI bei Patienten mit maligner Tumorerkrankung (42,6\% vs. 50,0\%, $p=0,032)$. Bei Unterscheidung zwischen hämoblastischen [Hämob. (+)/AKI (+)] und 


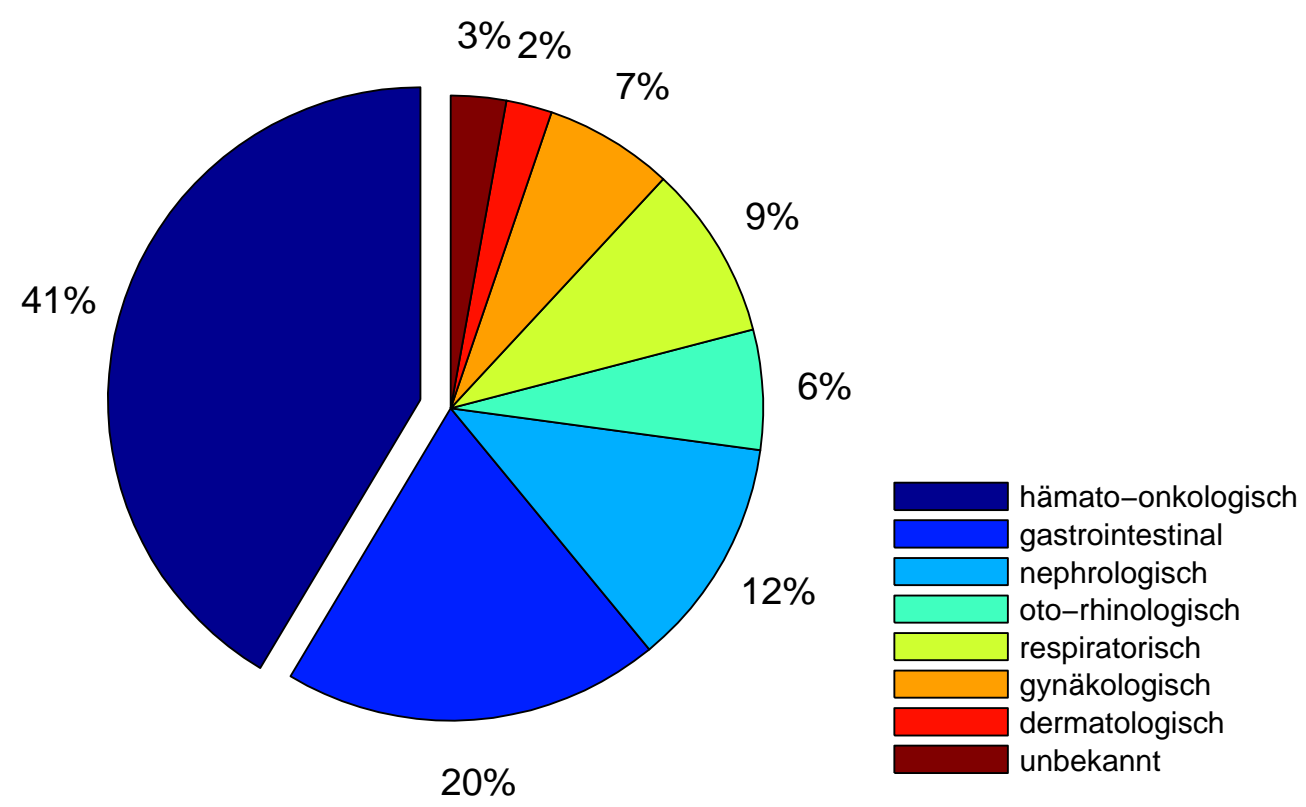

AbBiLdung 3.17: Verteilung maligner Neoplasien im Patientenkollektiv.

\begin{tabular}{|c||c|c|c|}
\hline \multicolumn{4}{|c|}{ Maligne Neoplasie } \\
\hline Merkmal & $\begin{array}{c}\text { Solide } \\
(n=123)\end{array}$ & $\begin{array}{c}\text { Hämoblastisch } \\
(n=87)\end{array}$ & $p$ \\
\hline Alter $(y)$ & $68,8 \pm 12,4$ & $58,1 \pm 13,5$ & $<0,001$ \\
Behandlungsdauer $(d)$ & $7,0 \pm 8,1$ & $9,4 \pm 8,6$ & 0,1 \\
SAPS II $(-)$ & $37,4 \pm 9,3$ & $40,9 \pm 10,3$ & $<0,01$ \\
Mortalität $n \mid(\%)$ & $41(33,3)$ & $35(40,2)$ & 0,25 \\
Geschlecht $(m) n \mid(\%)$ & $76(61,8)$ & $58(66,7)$ & 0,47 \\
AKI $n \mid(\%)$ & $55(44,7)$ & $50(57,5)$ & $<0,05$ \\
Sepsis $n \mid(\%)$ & $46(37,4)$ & $49(56,3)$ & $<0,01$ \\
Nierenersatztherapie $n \mid(\%)$ & $44(35,8)$ & $35(40,2)$ & 0,51 \\
Beatmung $n \mid(\%)$ & $53(43,1)$ & $44(50,6)$ & 0,28 \\
\hline
\end{tabular}

TABelle 3.7: Charakteristika der Patienten mit soliden und hämoblastischen Neoplasien. 


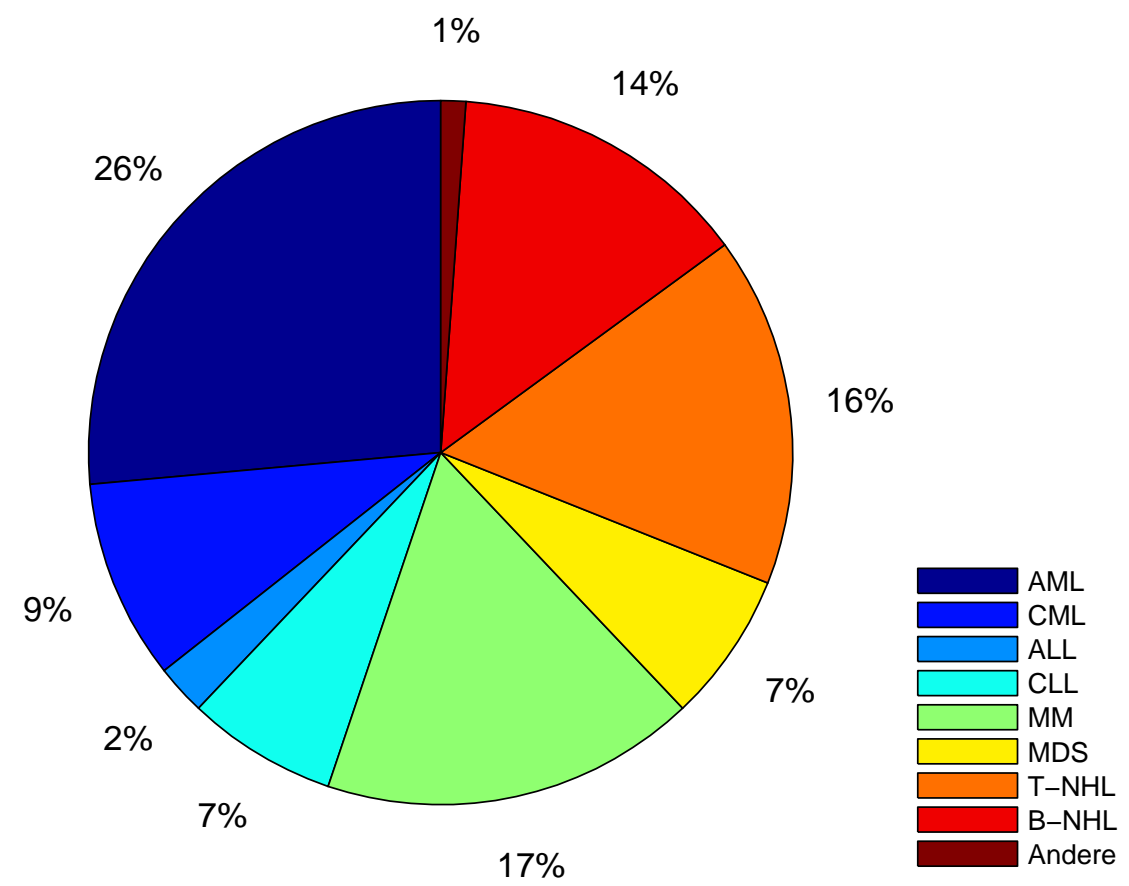

AbBildung 3.18: Verteilung der Hämoblastome im Patientenkollektiv. AML Akute myeloische Leukämie; CML Chronische myeloische Leukämie; ALL Akute lymphatische Leukämie; CLL Chronische lymphatische Leukämie; MM Multiples Myelom; MDS Myelodysplastisches Syndrom; T-NHL T-Zell Non-Hodgkin-Lymphom; B-NHL B-Zell Non-Hodgkin-Lymphom.

soliden Malignomen [Solide (+)/AKI (+)] zeigte sich eine schwach signifikante Präferenz des AKI bei hämoblastischen Malignomen (57, $5 \%$ vs. 44,7\%, $p=0,046)$.

Hinsichtlich der Mortalität ergaben sich ähnliche Zusammenhänge. Der Vergleich der Patienten [Malignom (-)/AKI (+)] vs. [Malignom (+)/AKI (+)] ergab eine höhere Mortalität bei Assoziation von Malignom und AKI (28,7\% vs. 51,4\%, $p<0,001)$.

Bei Unterscheidung zwischen Patienten mit hämoblastischem Malignom [Hämob. $(+) /$ AKI (+)] und Patienten ohne hämoblastisches Malignom [Hämob. (-)/AKI (+)] zeigte sich eine deutliche Erhöhung der Mortalität bei Assoziation des AKI mit hämoblastischen Tumorerkrankungen (60,0\% vs. 28,7\%, $p=<0$, 001). Die Mortalität bei Assozziation des AKI mit soliden Tumoren war gegenüber der von AKI mit hämoblastischen Tumoren nicht signifikant verschieden $(60,0 \%$ vs. $43,6 \%, p=0,069)$.

Zusammenfassend ist festzustellen, dass bei Komorbidität von Tumorerkrankungen und 

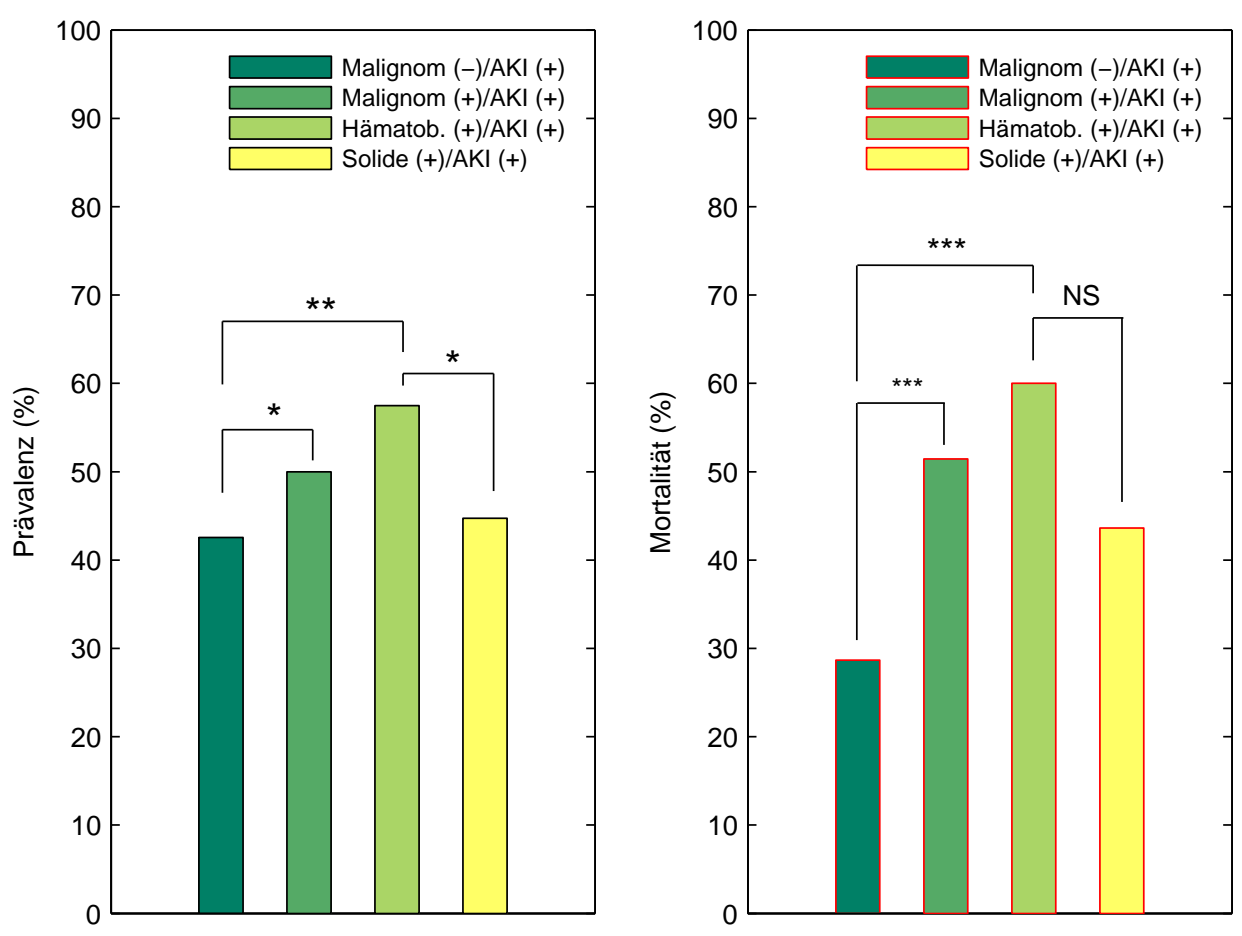

AbBiLDung 3.19: Prävalenz und Mortalität bei malignen Neoplasien und AKI.

AKI das Mortalitätsrisiko deutlich erhöht war, dies galt insbesondere bei hämoblastischen Erkrankungen.

\subsection{Akutes Nierenversagen bei Malignom und Sepsis}

Die Prävalenz und Mortalität in der Kombination von Malignom, Sepsis und AKI ist in Abb. 3.20 zusammengestellt. Die Prävalenz bei Komorbidität von Malignom, Sepsis und AKI [Malignom (+)/Sepsis (+)/AKI (+)] war deutlich gegenüber der Vergleichsgruppe [Malignom (+)/Sepsis (-)/AKI (+)] erhöht (71,6\% vs. 32,2\%, $p<0,001)$. Die Mortalität bei gleichen Vergleichsgruppen ist ebenfalls deutlich erhöht $(67,6 \%$ vs. $21,6 \%$, $p<0,001$ ). Mehr als $2 / 3$ der Patienten, die an einer malignen Neoplasie erkrankt waren und darüber hinaus eine Sepsis mit assoziiertem AKI entwickelten, waren im Zeitraum der Behandlungsdauer auf der ICU verstorben. 

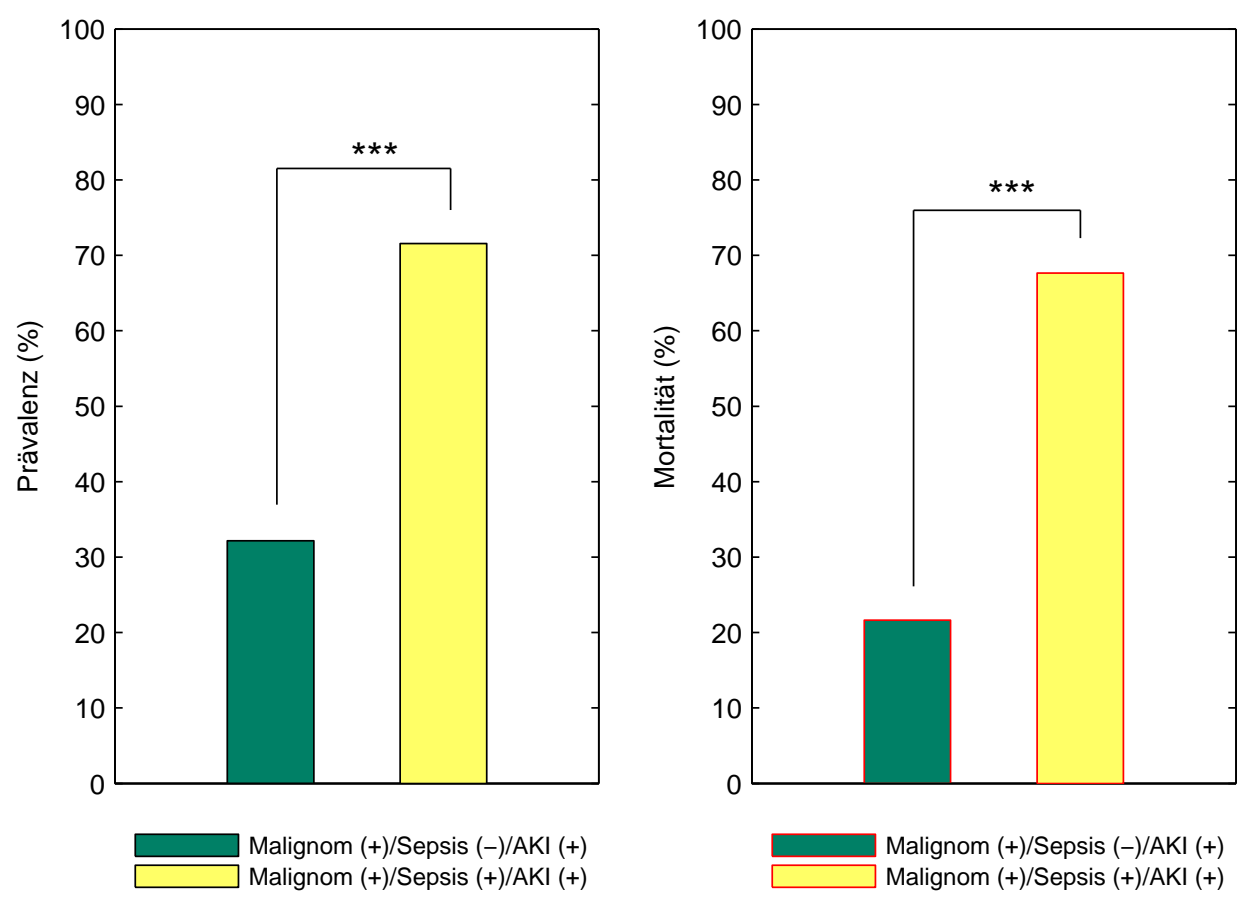

AbBildung 3.20: Prävalenz und Mortalität bei malignen Neoplasien, Sepsis und AKI.

\subsection{Nierenersatztherapie bei Malignom und Sepsis}

Unter dem Begriff Nierenersatztherapie werden in dieser Untersuchung alle gängigen Dialyse-Verfahren subsummiert (Hämodialyse, Hämodiafiltration). Es wurden im Beobachtungszeitraum insgesamt $\sim 1 / 3$ der Patienten (34,5\%) mit einem Dialyseverfahren behandelt. Bei 48,0\% der Erkrankten wurde die Dialyse im Verlauf eines AKI durchgeführt, bei 25,6\% im Rahmen einer akuten Dekompensation einer chronischen Niereninsuffizienz und bei $22,7 \%$ bei chronischer Niereninsuffizienz ohne Dekompensation.

Die besondere Betrachtung zur Prävalenz und Mortalität von Patienten mit Malignom und Sepsis unter den Bedingungen einer durchgeführten Nierenersatztherapie ist in Abb. 3.21 dargestellt. Die Prävalenz in den Untergruppen war wie folgt verteilt: [Malignom (-)/Sepsis (-)] 25, 8\%; [Malignom (-)/Sepsis (+)] 51,0\%; [Malignom (+)/Sepsis $(-)$ 28,7\%; [Malignom (+)/Sepsis (+)] 49,5\%. Die Unterschiede zwischen den dialysepflichtigen Malignom-Patienten mit assoziierter Sepsis waren im Vergleich zu den korrespondierenden Kontroll-Gruppen hochsignifikant $(p<0,001)$. 

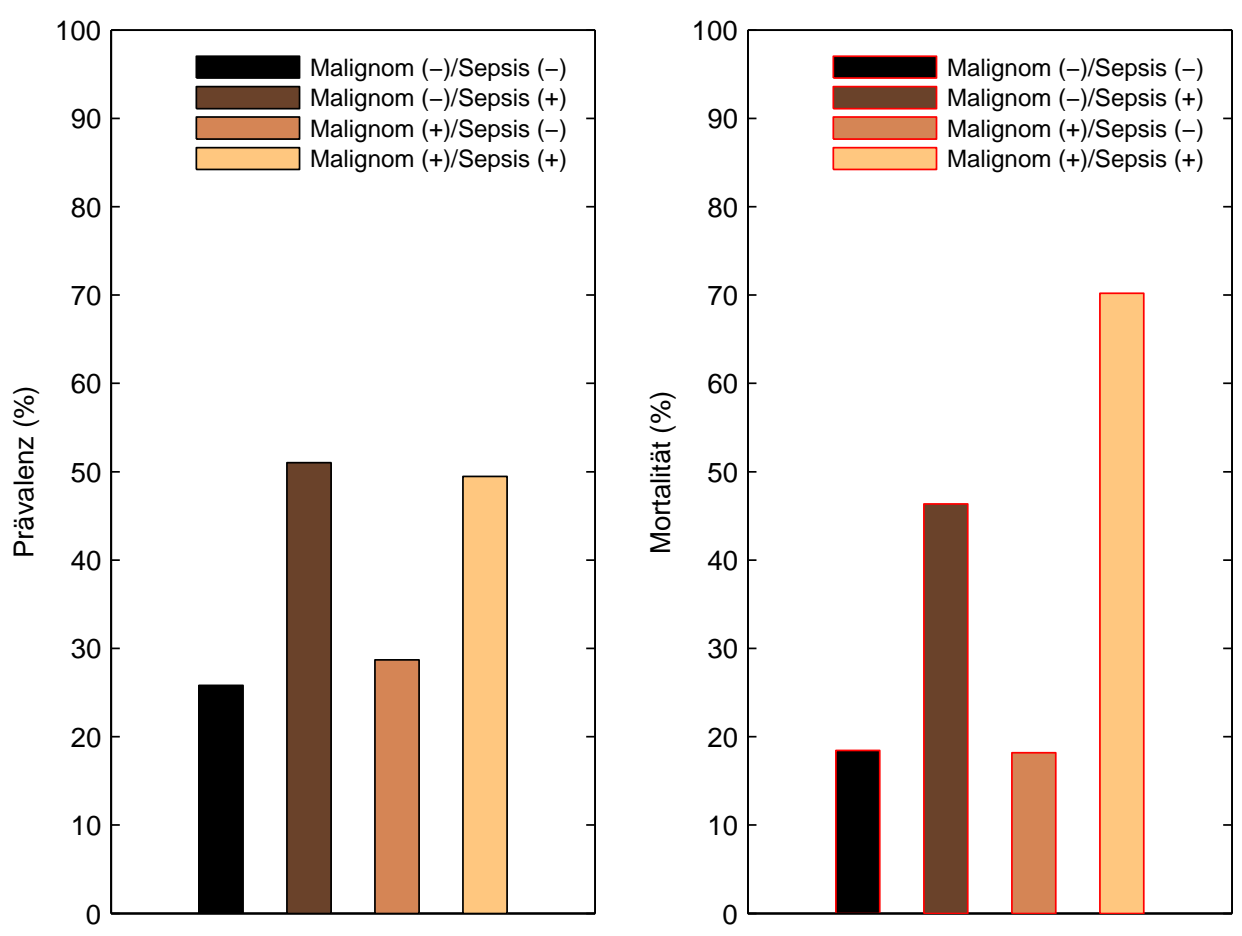

AbBiLDung 3.21: Häufigkeit und Mortalität bei malignen Neoplasien und Sepsis bei Nierenersatztherapie.

Auch im Hinblick auf die Mortalität war die Sepsis bei Malignom-Patienten unter Nierenersatztherapie ein dominierender Faktor. Bei [Malignom (-)/Sepsis (+)]-Patienten betrug die Mortalität 46,3\%, bei [Malignom $(+) /$ Sepsis $(+)$ ]-Patienten $70,2 \%(p=<0,01)$. Die Zahlenwerte ergeben, dass Prävalenz und Mortalität bei Patienten mit Nierenversagen und erforderlicher Nierenersatztherapie durch eine assoziierte Sepsis deutlich erhöht waren, dies allerdings unabhängig vom gleichzeitigen Vorliegen oder Fehlens einer malignen Tumorerkrankung als weiterer Komplikation.

Da sich bei den malignen Neoplasien mit Koprävalenz von Sepsis und AKI besonders ungünstige Konstellationen zeigten, wurde die Betrachtung der Mortalität auf solide Tumoren und Hämoblastosen erweitert (Abb. 3.22). Bei Koexistenz von Sepsis, dialysepflichtigen AKI und hämoblastischer Grunderkrankung [Sepsis (+)/AKI (+)/Dial. $(+) /$ Hämobl. (+)] betrug die Mortalität 90,5\% und überstieg die mit 52,9\% geringere Mortalität der Patienten mit solider Tumorerkrankung [Sepsis (+)/AKI (+)/Dial. $(+) /$ Solide $(+)],(p=0,02)$. In den korrespondierenden Vergleichsgruppen ohne Nierenersatztherapie [Sepsis $(+) /$ AKI $(+) /$ Dial. $(-) /$ Solide $(+)]$ vs. [Sepsis $(+) /$ AKI $(+) /$ Dial. 


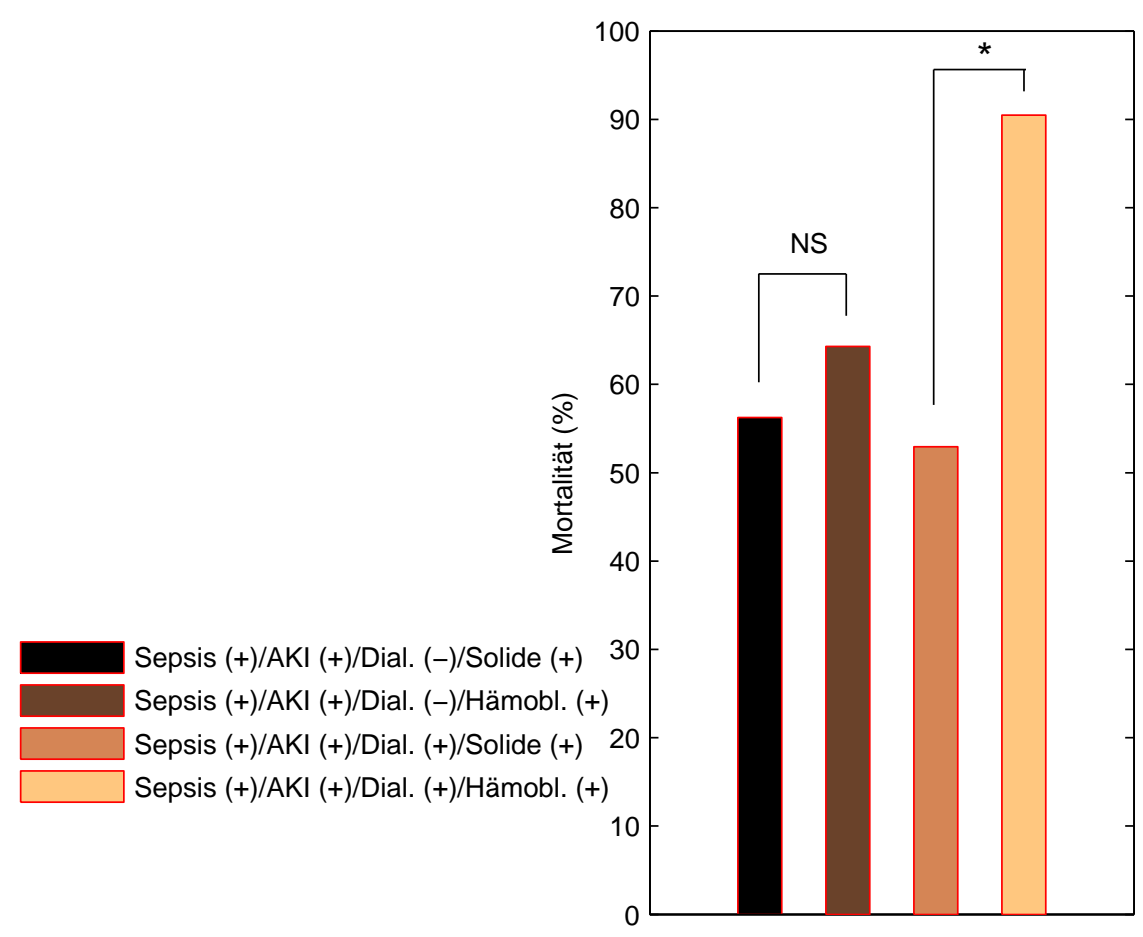

AbBiLdung 3.22: Mortalität bei Hämoblastosen und Sepsis bei Nierenersatztherapie.

(-)/Hämobl. (+)] war die Mortalität von der Art der bestehenden Neoplasie unabhängig $(56,3 \%$ vs. $64,3 \%, p=0,72)$.

\subsection{Akutes Nierenversagen bei Leberzirrhose}

Eine Leberzirrhose wurde bei 82 Patienten (8, 2\%; $m 57$ (69,5\%), w 25 (30,5\%)) diagnostiziert. Die häufigste Ätiologie der Leberzirrhose war alkoholtoxisch (48,8\%). Eine virusbedingte Leberzirrhose lag bei 12,2\% der Patienten vor. Bei 25 Patienten ließ sich keine Ursache der Leberzirrhose ermitteln. Die Behandlungsergebnisse und Risikofaktoren sind in Tab. 3.8 zusammengefaßt. 


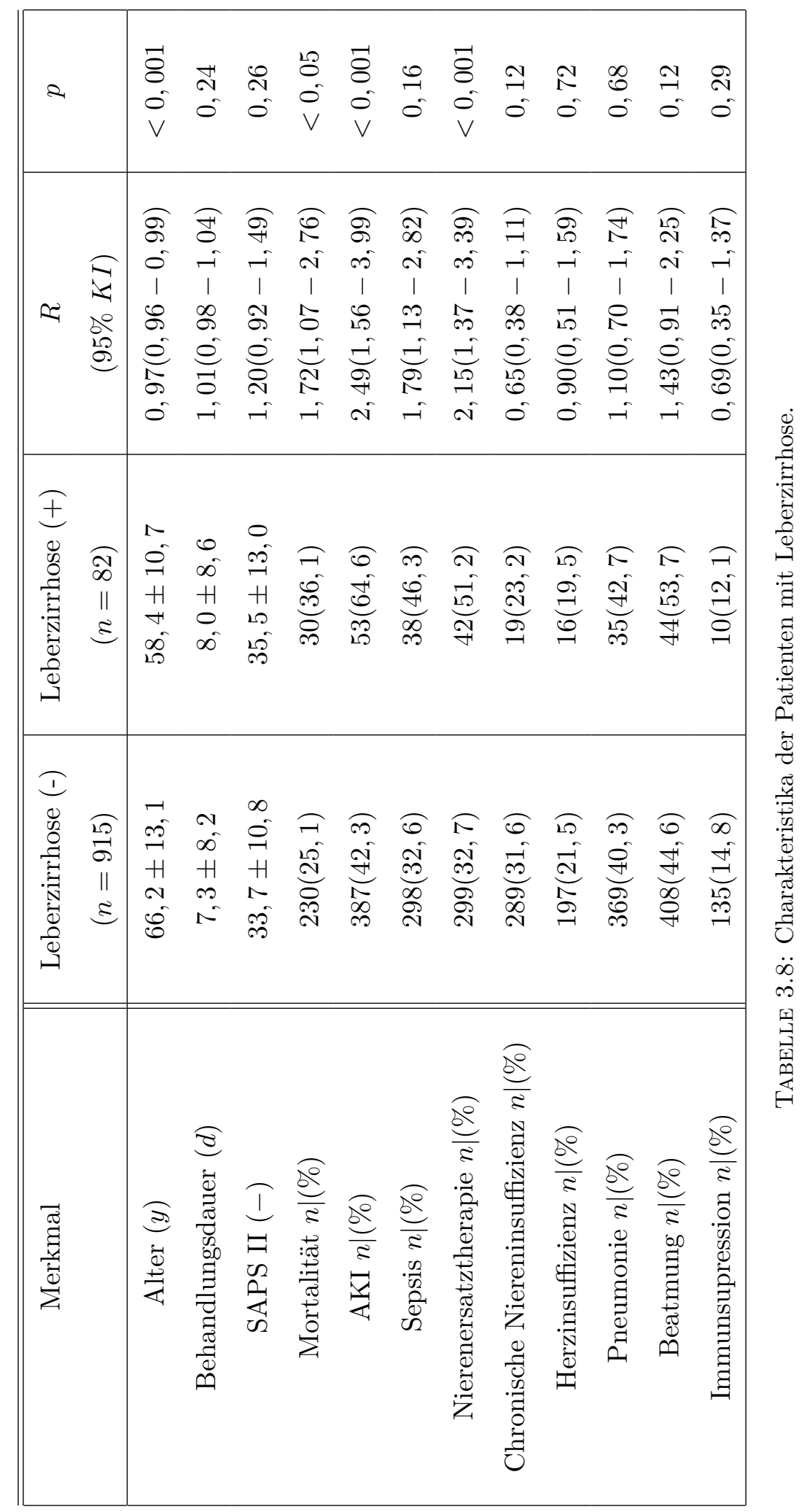




\subsection{Zusammenfassung der wichtigsten Ergebnisse}

In die Untersuchung wurden 997 Patienten einbezogen, die im 3-jährigen Behandlungszeitraum von 2009 - 2011 auf der ICU der UMG behandelt wurden.

- Die Prävalenz der betrachteten Haupterkrankungen AKI, Sepsis und maligne Neoplasien lag entsprechend bei $44,1 \%, 33,7 \%$ und $21,1 \%$.

- Die Mortalität bezogen auf das Gesamtkollektiv betrug 21,6\% $(n=260)$. Aufgeschlüsselt nach den Haupterkrankungen (s.o.) betrug die Mortalität 34, 1\%, 47, $0 \%$ und $36,2 \%$.

- Bei Patienten, die während der Behandlung auf der ICU ein AKI entwickelten (28,4\%), war die Mortalität deutlich erhöht $(46,4 \%)$.

- Die Mortalität bei der septischen Erkrankung stieg annähernd proportional mit dem Schweregrad von $12 \%-62 \%$.

- Die Koinzidenz des AKI bei septischer Grunderkrankung war mit 68,5\% außerordentlich hoch und betraf mehr als 2/3 der Patienten.

- Die Prävalenz einer Sepsis war bei Patienten mit Hämoblastosen gegenüber denen mit soliden Tumorerkrankungen deutlich erhöht (56,3\% vs. 37,4\%).

- Bei Komorbidität von Tumorerkrankungen und AKI war das Mortalitätsrisiko deutlich erhöht und betraf insbesondere hämoblastische Erkrankungen (60,0\%).

- Die Prävalenz bei Komorbidität von AKI, Sepsis und maligenen Neoplasien war mit 71,6\% deutlich erhöht. Die Mortalität war ebenfalls deutlich erhöht $(67,6 \%$. Mehr als 2/3 der Patienten waren im Zeitraum der Behandlungsdauer auf der ICU verstorben.

- Bei Koexistenz von dialysepflichtigen AKI, Sepsis und hämoblastischer Grunderkrankung betrug die Mortalität 90,5\%. 


\section{Kapitel 4}

\section{Diskussion}

In dieser Untersuchung wurden ingesamt 997 Patienten der nephrologischen Intensivstation der UMG erfaßt, die im 3-Jahreszeitraum von 2009 - 2011 behandelt wurden. Der Schwerpunkt der Betrachtung war auf das AKI in Konnotation mit Sepsis und malignen Neoplasien gerichtet.

Die Arbeit stellt nur die Behandlungsergebnisse eines einzelnen intensivmedizinischen Zentrums (zentrisch) dar; es handelt sich um eine retrospektive Untersuchung. Die Ergebnisse sind nicht ohne weiteres auf andere intensivmedizinische Bereiche übertragbar. Die Behandlungsschwerpunkte betrafen überwiegend das AKI (44,6\%), gefolgt von Sepsis $(34,1 \%)$ und malignen Neoplasien $(21,3 \%)$. Insoweit muß davon ausgegangen werden, dass im Krankengut bezüglich der Verteilung der Haupterkrankungen eine spezialisierte Selektion vorlag und eine Normalverteilung in Bezug auf Krankheitshäufigkeiten nicht gegeben war. Für die Ergebnisse dieser Beobachtungsstudie ist besonders auf die Bedeutung des sog. Selektionsbias hinzuweisen. Durch eine ungewollte Selektion von Patienten können bereits vor Untersuchungsbeginn systematische Unterschiede zwischen den Gruppen, die miteinander verglichen werden, entstanden sein. So kann ein Unterschied in den Behandlungsergebnissen lediglich darauf beruhen, dass in einem Patientenkollektiv oder in Untergruppen dieses Kollektivs Patienten mit schwereren Erkrankungsformen oder ausgeprägterem Risikoprofil beobachtet wurden. Im Ergebnis sind dann die sich aus Zahlenwerten ergebenden Unterschiede, z.B. hinsichtlich Prävalenz und Mortalität, lediglich scheinbare, virtuelle Unterschiede. Mit großer Wahrscheinlichkeit darf angenommen werden, dass ein Selektionsbias im Krankengut der ICU der UMG vorliegt. Dies ist allein aufgrund der Prävalenz der Haupterkrankungen AKI, Sepsis und maligne Neoplasien anzunehmen (Abb. 3.3). Diese Effekte werden aber durch relativierende Angaben (in \%) zu Prävalenz und Mortalität nur scheinbar eliminiert. 
$\mathrm{Zu}$ berücksichtigen ist ferner, dass es sich bezüglich der Grunderkrankungen der Patienten um ein sehr inhomogenes Kollektiv handelt und Nierenfunktionsstörungen im engeren (AKI) oder weiteren (Niereninsuffizienz, Dialyse) Sinne den Grund zur Aufnahme auf eine nephrologische Spezial-ICU gaben (Abb. 3.2). Der anzunehmende Selektionsbias, der durch die Art der Zuweisung auf die ICU bedingt ist, ist aber für die Ergebnisse dieser Untersuchung nicht als nachteilig zu werten, er ergibt sich zwangsläufig aus der Verteilung der Patientencharakteristika und dem Untersuchungszeitraum von 3 Jahren. Nur der Vollständigkeit halber sei erwähnt, dass seitens des Untersuchers kein Einfluß auf die Auswahl der Patienten genommen wurde (abgesehen von der Herausnahme von 20 Patienten wegen unzureichender Dokumentation oder Alter).

Es wird auch davon ausgegangen, dass alle Patienten optimal intensivmedizinisch behandelt wurden, unabhängig davon, ob der individuelle Behandlungserfolg positiv (Entlassung von der ICU) oder negativ (Versterben auf der ICU) war. Diese Annahme gilt gleichfalls für alle Vergleichsuntersuchungen aus der Literatur, soweit diese in die Betrachtung einbezogen wurden. Bei der Therapie des AKI wurden die standardisierten Behandlungsrichtlinien (Kidney Disease: Improving Global Outcomes, KDIGO, 2012) beachtet und mit spezifischen Therapiemaßnahmen der Grundkrankheit ergänzt. Es bedarf auch des ergänzenden Hinweises, dass im Betrachtungszeitraum von 2009 - 2011 die grundsätzliche Behandlungsstrategie und die medizinische Versorgung auf der ICU vermeintlich unverändert waren (s. Tab. 3.1).

Bei den Angaben zu Quotenverhältnissen wird ein vermuteter Risikofaktor angegeben, der besagt, wie stark ein Zusammenhang mit einer bestimmten Erkrankung besteht. Das Quotenverhältnis ist eine assoziative Kenngröße, aus der sich keine tatsächliche Kausalität ableiten läßt. Weiterhin ist zu berücksichtigen, dass es sich um eine retrospektive Untersuchung und keine randomisierte Fall-Kontroll-Studie handelt. Die Fallzahlen sind jedoch hinreichend groß und der Untersuchungszeitraum von 3 Jahren hinreichend bemessen, so dass auch bei der Betrachtung von Untergruppen statistisch valide Ergebnisse erzielt werden konnten. In dieser Untersuchung werden nur die kurzzeitigen Behandlungsergebnisse von Patienten über den Zeitraum der Behandlung auf der ICU betrachtet. In Ermangelung des Zugangs zu Patientendokumentationen außerhalb der UMG, konnte der weitere Krankheitsverlauf nach der Entlassung von der ICU und somit die Frage der Inzidenz von Folgeerkrankungen (chronische Niereninsuffizienz, dialysepflichtiges Nierenversagen), Spätkomplikationen, Risikofolgen und Spät-Mortalität nicht behandelt werden. 


\subsection{Diagnose des AKI}

In der Diagnostik des AKI werden als Kriterien die dynamischen Veränderungen der Kreatinin-Konzentration im Serum $\left(\left[\Delta \mathrm{Kr}_{s}\right]\right)$ und der ausgeschiedenen Urinmenge $(\dot{U})$ herangezogen. Mit den AKIN-Empfehlungen zur Diagnose des AKI hat sich in den vergangenen 10 Jahren ein Kriterien-Standard herausgebildet, der international gemeinhin Verwendung findet (Tab. 2.2). Diese diagnostischen Kriterien wurden im Zuge der Behandlung der Patienten auf der ICU durchgehend angewendet. Eine kritische Durchsicht der Patientendokumentationen ergab, dass die Diagnose des AKI entsprechend der Klassifizierung stets zutreffend und zeitgerecht, d.h. nicht verzögert, erfolgte. Mit dem Hinweis auf die Zeitgerechtigkeit der Diagnosestellung ist aber nicht gesagt, dass die Diagnose eines bereits bestehenden AKI oder eines sich entwickelnden AKI stets hinreichend frühzeitig gestellt wurde. Die Fokussierung auf die dynamischen Veränderungen der Kreatinin-Konzentration $\left(\left[\Delta \mathrm{Kr}_{s}\right]\right)$ als Biomarker des AKI ist bezüglich Sensibilität und Spezifität mit gewissen Einschränkungen zu betrachten. Mit der Definition des AKI nach den AKIN-Kriterien (s. Tab. 2.2, Mehta et al. 2007) wird ein AKI bei Überschreiten des $48 \mathrm{~h}$-Zeitfensters nicht erfaßt. Außerdem sind zur Diagnose des Stadium III insgesamt 3 zu erfüllende Kriterien erforderlich $\left(\left[\Delta \mathrm{Kr}_{s}\right], \dot{U}\right.$ und die Erfordernis einer Nierenersatztherapie). Die AKIN-Kriterien zur Diagnose des AKI enthalten eine gewisse intrinsische Unschärfe insoweit, als auf akute relative Veränderungen der $\left[\mathrm{Kr}_{s}\right]\left(\left[\Delta\left[K r_{s}\right]_{r e l}\right]\right)$ innerhalb eines definierten Zeitintervalls bezogen auf einen Basis-Ausgangswert Bezug genommen wird. Dies ist aus praktischen Erwägungen heraus begründet, weil verläßliche Ausgangswerte der $\left[\mathrm{Kr}_{s}\right]$ bei Aufnahme auf die ICU häufig nicht vorliegen. Die Einbeziehung einer statischen Komponente für die Diagnose des AKIN-Stadium III, wobei der aktuelle Absolutwert der Kreatinin-Konzentration $\left[\mathrm{Kr}_{s}\right]$ zum Aufnahmezeitpunkt betrachtet wird, ist deshalb sinnvoll, da keine retrospektive Beurteilung der dynamischen Entwicklung von $\left(\left[\Delta \mathrm{Kr}_{s}\right]\right)$ mangels vorliegender Laborwerte möglich ist. Diese diagnostische Unschärfe kann aber zu einer Über- oder Unterschätzung des AKI hinsichtlich zeitlicher Entwicklung und Schweregrad führen (Siew et al. 2010).

Grundsätzlich besteht unter physiologischen Bedingungen ein Gleichgewicht zwischen der Kreatinin-Produktion in der Skelettmuskulatur und der Kreatinin-Exkretion (steady state). Damit hängt die $\left[\mathrm{Kr}_{s}\right]$ auch von der Muskelmasse ab, d.h. von der physischen Konstitution des Patienten. Dies gilt insbesondere für kachektische Patienten oder Diabetiker mit geringer Muskelmasse. Normale $\left[\mathrm{Kr}_{s}\right]$ schließen eine eingeschränkte Nierenfunktion jedoch nicht aus, da erst aufgrund der nicht-linearen Beziehung zwischen glomerulärer Filtrationsrate und $\left[\mathrm{Kr}_{s}\right]$ bei einer Einschränkung der glomerulären Filtrationsrate auf $<50 \%$ ein Anstieg der $\left[\mathrm{Kr}_{s}\right]$ zu erwarten ist. Weiterhin ist die Kreatinin-Produktion von der Kreatinin-Synthese in der Leber abhängig, die bei Lebererkrankungen und anderen 
endokrinen Erkrankungen reduziert sein kann. Da die laborchemische Untersuchung im Blutserum erfolgt, ist die Flüssigkeitsbilanz des Organismus zu beachten, da z.B. Patienten mit Herzinsuffizienz häufig eine verstärkte Flüssigkeitsretention aufweisen. Diese Umstände können dazu führen, dass die Inzidenz des AKI verzögert und nicht zeitgerecht erkannt wird. Die Häufigkeit einer verzögerten Diagnose ohne Korrektur der $\left[\mathrm{Kr}_{s}\right]$ unter Berücksichtigung der Flüssigkeitsbilanz betraf nach Angaben aus der Literatur $19 \%$ der Patienten mit AKI (Liu et al. 2011). Andererseits konnte bei Patienten mit herzchirurgischen Eingriffen gezeigt werden, dass eine mäßige $\left[\Delta \mathrm{Kr}_{s}\right]$-Erhöhung nicht notwendigerweise ein Zeichen einer Nierenparemchym-Schädigung darstellt insoweit, als tubuläre Biomarker unter diesen Umständen unverändert sein können (Coca et al. 2014). Diese Ergebnisse geben Anlaß zu Bemühungen, sowohl Indikatoren für Funktionsveränderungen $\left(\left[\Delta \mathrm{Kr}_{s}\right]\right.$ und $\left.\dot{U}\right)$ als auch Indikatoren für Strukturveränderungen (Biomarker für Tubulusschädigungen) zur Diagnose des AKI heranzuziehen (McCullough et al. 2013; Basu et al. 2014; Kellum et al. 2014; Siew und Davenport 2015). Neuere experimentelle und klinische Untersuchungen haben weitere Erkenntnisse über die Pathophysiologie des AKI erbracht. So wird in der Genese des AKI ein renaler Ischämie-Reperfusionsschaden nicht mehr als ausschließliche conditio sine qua non angesehen. Eine Einschränkung der Nierenfunktion kann somit auch ohne tubuläre Schädigung oder Nekrose eintreten.

\subsection{Prävalenz und Mortalität des AKI}

Über die Inzidenz des AKI gibt es keine verläßlichen Angaben. Die Gründe hierfür sind vielfältiger Natur. Zum einen ist dies bedingt durch den Umstand, dass über Jahrzehnte eine vereinheitlichte Definition des AKI mit standardisierten diagnostischen Kriterien nicht vorlag, zum anderen bedingt durch die sehr unterschiedliche Zusammensetzung der betrachteten Patientenkollektive. Eine zusammenfassende Übersicht zur Epidemiologie des AKI wurde kürzlich publiziert (Siew und Davenport 2015). Einer neueren Untersuchung zufolge, beträgt die Inzidenz des AKI bei weltweiter Betrachtung (154 Studien, $n=3.585 .911) 21,6 \%$ bei Erwachsen und 33,7\% bei Kindern, die Mortalität 23,9\% bei Erwachsenen und 13, 8\% bei Kindern (Susantitaphong et al. 2013). Hierbei ist allerdings zu berücksichtigen, dass Ergebnisse aus westlich-orientierten Industrieländern sowie aus Entwicklungsstaaten einbezogen wurden.

Älteren Untersuchungen zufolge beträgt die Inzidenz des AKI auf einer ICU $1-25 \%$, die Mortalität 15 - 60\% (Liano und Pascual 1996; Brivet al al. 1996; Uchino et al. 2005b). Basierend auf einer multizentrischen ICU-Untersuchung $(n=120.123)$ in Australien im Zeitraum von 2000 - 2005 betrug die Inzidenz des AKI 36,1\%, die Mortalität mit den 3 Stadien des AKI ansteigend 7,9\% - 33, 2\% (Bagshaw et al. 2008a). In einer ähnlichen 
unizentrischen Untersuchung $(n=5.383)$ betrug die Inzidenz $67 \%$, die Mortalität in Abhängigkeit der Stadien des AKI 8, 8\% - 26, 3\% (Hoste et al. 2006). Weiterhin betrug die AKI-Inzidenz bei einer multizentrischen ICU-Untersuchung $(n=41.972) 35,8 \%$, die Mortalität stadienabhängig 20,9\%, 45,6\% und 56,8\% (Ostermann und Chang 2007). Andere Ergebnisse (multizentrisch, $n=2.164$ ) waren: Inzidenz 10, 8\%, Mortalität 20, 0\%, $29,3 \%$ und 49,5\% (Cruz et al. 2007). In einer finnischen Untersuchung (multizentrisch, $n=2.901$ ) lag die Inzidenz bei Patienten auf der ICU bei 39, 3\%, nach den AKIN-Stadien I-III bei $17,2 \%, 8,0 \%$ und 14,1\%. Die Mortalität auf der ICU lag bei 25,6\% (Nisula et al. 2013). Die Übersicht, die Untersuchungen erfaßt, in denen bereits die Klassifizierung des AKI nach den standardisierten AKIN-Kriterien vorgenommen wurde, zeigt trotz vereinheitlichter Klassifizierung erheblich voneinander abweichende Angaben.

In der hier vorliegenden Untersuchung lag die Prävalenz des AKI $(n=440 / 997)$ im Patientenkollektiv bei 44,1\%. Zum Zeitpunkt der Aufnahme auf die ICU hatten 315 (71,6\%) Patienten bereits ein AKI, 125 (28,4\%) Patienten entwickelten ein AKI während der Behandlung auf der ICU. Im Vergleich zu den Prävalenz-Angaben aus der korrespondierenden Literatur (s.o., im Mittel 38\%, Spannweite 26,3\% - 56.8\%) lag die Prävalenz des AKI mit 44,1\% des hier untersuchten Patientenkollektivs leicht oberhalb des Mittelwerts vergleichbarer Untersuchungen. Eine einfache Erklärung für dieses Mißverhältnis ist nicht ohne weiteres erkennbar. Hier ist erneut auf die Bedeutung des sog. Selektionsbias (s.o.) hinzuweisen insofern, als die Patienten auf der nephrologischen ICU der UMG ein nach nephrologisch oriententierten Behandlungserfordernissen vorselektiertes Patientenkollektiv einer nephrolologisch spezialisierten ICU waren. Der Vergleich der Behandlungserfolge darf nicht überbewertet werden und muß auf die Feststellung reduziert werden, dass die Größenordnung der Prävalenz des AKI bei der Vergleichsbetrachtung mit den Ergebnissen anderer veröffentlichter Untersuchungen grosso modo ähnlich war. Gerade die hohe Prävalenz von 44,1\% ist als Hinweis zu werten, dass das hier untersuchte Patientenkollektiv mit einem Selektionsbias behaftet war, es sich somit um eine nach höheren Werten verschobene Scheinprävalenz handelt - also nicht als epidemiologisch repräsentativ für eine größere geographisch umrissene Bevölkerungscharakteristik anzusehen ist.

$\mathrm{Zu}$ berücksichtigen ist ferner, dass es sich bei vergleichenden Betrachtungen der Ergebnisse des vorliegenden Patientenkollektivs mit denen anderer Untersucher aus Angaben in der Literatur um einen Vergleich unizentrischer Behandlungsergebnisse mit denen multizentrischer Behandlungsergebnisse handelt. Bei multizentrischen Untersuchungen ist gemeinhin anzunehmen, dass abweichende Behandlungsergebnisse (etwa wie die der vorliegenden Untersuchung) von auf bestimmte Erkrankungen spezialisierten 
ICU-Einrichtungen bei kumulativer Zusammenfassung (etwa bei Einschluß der Ergebnisse dieser unizentrischen Untersuchung in eine multizentrische Studie) von Behandlungsergebnissen "herausgemittelt", d.h. attenuiert werden.

Es muß auch darauf hingewiesen werden, dass - ungeachtet einer diagnostischen Standardisierung des AKI nach den AKIN-Kriterien - eine Vergleichsbetrachtung der Behandlungergebnisse des AKI nur dann eine gemeinsame Basis haben kann, wenn die Prävalenz des AKI auf vergleichsweise identische Grunderkrankungen (Herzinsuffizienz, Sepsis, maligne Neoplasen etc.) bezogen wird. Dies ist jedoch in sämtlichen Vergleichsuntersuchungen nicht der Fall. Auf die Besonderheit des AKI als Syndrom bei einer bestehenden Grundkrankheit wurde bereits in der Einleitung zu dieser Untersuchung hingewiesen (s. Kap. 1.3).

Im untersuchten Patientenkollektiv lag gemäß der AKIN-Klassifizierung bei 18,1\% der Patienten ein Stadium I vor, 9, $4 \%$ befanden sich im Stadium II, 72,5\% im Stadium III. Die Zahl der nach Stadium III klassifizierten Patienten muss nach den AKI-Kriterien um die Zahl derjenigen Patienten ergänzt werden, die aufgrund einer bestehenden chronischen Niereninsuffizienz dialysepflichtig waren und zwar unabhängig davon, ob diese tatsächlich ein AKI entwickelten oder nicht. Insgesamt lag bei 45, 9\% der Patienten ein AKIN-Stadium III vor. Diese Zahlenangaben zur Verteilung des AKI auf die AKINStadien sind deutlich abweichend und höher als die anderer Untersuchungen (Nisala et al. 2013; Porter et al. 2014). Auch hier dürfte der Grund für die Abweichungen auf den Selektionsbias des Patientenkollektivs der ICU der UMG zurückzuführen sein.

Die Mortalität bei den Patienten, die bereits mit einem vorbestehenden AKI zur Aufnahme auf die ICU kamen, betrug 29,2\%. Patienten, die erst auf der ICU ein AKI entwickelten, hatten eine Mortalität von 46,4\%. Die Inzidenz der Entwicklung eines AKI während der Behandlung auf der ICU führte zu einem Anstieg der Mortalität um 16,7\%. Die Gesamtmortalität aller Patienten mit AKI betrug 34,1\%. Nach den AKINStadien I - III geordnet, betrug die Mortalität mit den Stadien ansteigend 22, 6\%, 31, 3\% und 35,5\%. Zum Vergleich betrug die Mortalität des AKI auf der ICU nach publizierten Untersuchungen im Mittel 38\% (Spannweite 26.3\% - 56.8\%, Hoste et al. 2006; Ostermann und Chang 2007; Cruz et al. 2007; Bagshaw et al. 2008a; Nisula et al. 2013). Der Behandlungserfolg des AKI der ICU der UMG ist daher in Bezug auf die Mortalität mit denen anderer Institutionen vergleichbar.

Die Behandlungsdauer der Patienten mit AKI war deutlich verlängert: 6,1 vs. 9, 0 Tage (s. Tab. 3.3 und Abb. 3.10). 


\subsection{Sepsis und AKI}

Vom Krankheitsbild der Sepsis ist $\sim 1 / 5$ aller Patienten auf einer ICU betroffen. Die Inzidenz des Sepsis-assoziierten AKI auf der ICU wird in der Literatur mit 11\% - 78\% angegeben (Hoste et al. 2003; Yegenaga et al. 2004; Lopes et al. 2007; Bagshaw et al. 2007; Cruz et al. 2007; Daher et al. 2007; Bagshaw et al. 2009; Alkandari et al. 2011; Sood et al. 2014). Die Kombination von AKI und Sepsis ist mit einer Mortalität von $\sim 70 \%$ verbunden im Vergleich zu 34,1\% bzw. 38\% bei Patienten, die allein an einem AKI erkrankten (s.o.). Die Kombination von Sepsis und AKI stellt daher in der Intensivmedizin ein gravierendes Problem dar, insbesondere auch dadurch, dass sich das Sepsisassoziierte AKI in seiner Entwicklung sehr schnell innerhalb weniger Stunden entwickelt (Schrier et al. 2004; Bagshaw et al. 2009). Die Pathphysiologie des AKI bei Sepsis ist komplex und multifaktoriell, verbunden mit hämodynamischen Veränderungen, endothelialer Fehlfunktion, Infiltration durch Entzündungszellen in das Nierenparenchym, intraglomeruläre Thrombose, Tubulusobstruktion, Nekrose und Aktivierung von pround antinflammatorischen Kaskaden (Wan et al. 2008; Bagshaw et al. 2009; Zarjou und Agarwal 2011; Alobaidi et al. 2015). Die pathophysiologischen Mechanismen des Sepsisassoziierten AKI sind von denen des AKI ohne Sepsis verschieden (Wan et al. 2008). Eine spezifische Therapie steht nicht zur Verfügung, die Therapie beschränkt sich auf eine schnelle Korrektur des Flüssigkeitshaushalts, den frühen Einsatz von Antibiotika und ggf. den frühen Einsatz einer Nierenersatztherapie.

Die Inzidenz des AKI korreliert mit der Schwere der Sepsis (4,2\% bei Sepsis, 22,7\% bei schwerer Sepsis, 52,8\% bei septischem Schock; Lopes et al. 2009 - zur SepsisKlassifikation s. Kap. 2.5.2). Auch besteht ein Zusammenhang zwischen Schwere der Sepsis und Schwere des AKI mit einem Anstieg des Einsatzes von notwendigen Nierenersatzverfahren von $24 \%$ auf $89 \%$ bei Fortschreiten der Erkrankung von Sepsis zum septischen Schock (Rangel-Frausto et al. 1995). Insgesamt ist die Prognose des Sepsisassoziierten AKI wesentlich ungünstiger als beim unifaktoriellen AKI als alleiniger Erkrankung (Bagshaw et al. 2007, 2009; Hoste et al. 2003; Oppert et al. 2008). Die Mortalität wird in der Literatur mit Werten von 49\% - 72\% angegeben (Neveu et al. 1996; Hoste et al. 2003; Yegenaga et al. 2004; Bagshaw et al. 2007, 2008b, 2009, Oppert et al. 2008; Lopes et al. 2009; Plataki et al. 2011).

Die Prävalenz einer Sepsis auf der ICU betrug 33,7\% im Gesamtkollektiv (s. Kap. 3.7, Abb. 3.12). Nach den Kriterien der Internationalen Sepsis-Konferenz (s. Kap. 2.5.2) lag bei den Sepsis-Patienten nach dem Schweregrad aufgeschlüsselt folgende Verteilung der Verlaufsformen vor: SIRS 7,4\%, Sepsis 44,0\%, schwere Sepsis 16,4\%, septischer Schock 32,1\%. Innerhalb des Sub-Kollektivs der Patienten mit Sepsis, verlief die septische Erkrankung bei 31,5\% ohne die zusätzliche Komplikation eines assoziierten AKI. Dagegen 
war bei mehr als $2 / 3$ der Patienten der septische Krankheitsverlauf mit einem AKI assoziiert $(68,5 \%)$. Hierbei war das AKIN-Stadium III mit 77,1\% am häufigsten vertreten.

Die Mortalität des Sepsis-assoziierten AKI lag im hier untersuchten Patientenkollektiv bei 51,7\% - mehr als die Hälfte der Patienten verstarben. Auch wenn sich die Mortalitätsziffer vergleichsweise innerhalb des weiten Rahmens der Literaturangaben bewegt (s.o.), ist der Behandlungsverlauf als unbefriedigend zu bewerten. Die Mortalität in den nach Schweregraden eingeteilten Sub-Klassen der Sepsis war mit dem Schweregrad des septischen Verlaufs ansteigend: SIRS 12\%, Sepsis 40\%, schwere Sepsis 55\%, septischer Schock $62 \%$. Hieraus wird ersichtlich, dass die Mortalität vorherrschend von den schweren Verlaufsformen der Sepsis bestimmt wurde. Etwas abweichend von der aus den Literaturangaben zu entnehmenden Tendenz der Zunahme der Mortalität mit gleichläufig zunehmender Schwere des AKI (Stadien I-III) und der Sepsis (Sepsis-Klassifizierung) war der Befund, dass bei unterschiedlicher Prävalenz der AKIN-Stadien I-III, keine signifikanten Unterschiede in der Mortalität bestanden (AKIN I/II 59, 1\%, AKIN III 48,6\%). Eine Erklärung hierfür drängt sich angesichts der Komplexität des Sepsis-assoziierten AKI nicht auf.

Die häufigste Begleiterkrankung der Sepsis war die Pneumonie (60,4\%). Eine Nierenersatztherapie war mit 50,3\% doppelt so häufig erforderlich wie bei Patienten ohne Sepsis. Der Vorteil der Nierenersatztherapie - insbesondere bei frühem Einsatz - ist im Hinblick auf die Mortalität und Verminderung von Spätfolgen umstritten (Ronco et al. 2000; Bagshaw et al. 2008c; Palevsky et al. 2008; Bellomo et al. 2009; Sutherland et al. 2010; Karvellas et al. 2011).

\subsection{Maligne Neoplasien und AKI}

Die Betrachtung maligner Neoplasien in Zusammenhang mit dem Auftreten eines AKI oder die Frage der Bedeutung der Komorbidität von Malignom, Sepsis und AKI hat in der veröffentlichten Literatur bisher nur einen geringen Raum eingenommen. Eine erste Übersicht zur Epidemiologie von AKI und Krebserkrankungen (Onko-Nephrologie) wurde unlängst publiziert (Lam und Humphreys 2012). Zwei gegenläufige Entwicklungen werden erkennbar. Zum einen ist die Inzidenz des AKI bei bestimmten Tumorerkrankungen besonders ausgeprägt (Nierenkarzinom, Multiples Myelom, Myelomniere (cast nephropathy), Leberkarzinom), zum anderen haben die positiven therapeutischen Erfolge beim Nierenzellkarzinom (partielle Nephrektomie) und multiplem Myelom die Überlebenschancen bei diesen Erkrankungen wesentlich verbessert. Dagegen sind Patienten mit akuter myeloischer Leukämie und akuter lymphatische Leukämie infolge der Nephrotoxizität der Chemotherapie einem besonders hohen Risiko der Entwicklung eines AKI 
ausgesetzt (Lameire 2014). Die Mortalität bei akuter myeloischer Leukämie und myelodysplastischem Syndrom bei dialysepflichtigem AKI betrug 62\% (Lahoti et al. 2010). Ein besonders hohes Risiko für die Entwicklung eines AKI besteht auch bei Patienten nach hämatopoetischer Stammzell-Transplantation (Humphreys 2010; Lopes und Jorge 2011). Auch ist die (nach Ansicht des Verfassers ethisch bedenkliche) Frage nach der Sinnfälligkeit einer Dialysetherapie bei AKI-Patienten mit infauster Prognose im Endstadium von Krebserkrankungen aufgeworfen worden (Darmon et al. 2007; Salahudeen et al. 2009).

Die Inzidenz des AKI bei malignen Neoplasien wurde mit 12\% angegeben (Salahudeen et al. 2013). Dagegen war die Prävalenz des AKI in der untersuchten Untergruppe der Patienten mit Malignom deutlich erhöht (50,0\%). Bei Unterscheidung zwischen hämoblastischen und soliden Malignomen zeigte sich eine schwach signifikante Präferenz des AKI bei hämoblastischen Tumorerkrankungen (57,5\% vs. 44,7\%).

Ähnliche Beziehungen ergaben sich für die Mortalität. Bei Komorbidität von Malignom und AKI betrug die Mortalität 51,4\%. Bei Assoziation von hämoblastischen Tumorerkrankungen und AKI war die Mortalität weiter auf 60,0\% gesteigert; ein Unterschied zu einer tendenziell geringeren Mortalität $(43,6 \%)$ bei soliden Tumoren und AKI war angedeutet, aber nicht signifikant. Als Ergebnis bleibt, dass bei Komorbidität von Malignom und AKI die Mortalität bei 50\% liegt und bei hämato-onkologischen Malignomen überwiegt. Diese Befunde stehen etwas im Gegensatz den Ergebnissen einer früheren Untersuchung in außeruniversitären ICU-Behandlungseinrichtungen, in der die Mortalität auf der ICU zwar 78\% betrug, die Erkrankung an einer malignen Neoplasie aber kein unabhängiger Faktor für die hohe Sterblichkeit war (Maccariello et al. 2011). Nach den Ergebnissen einer brasilianischen Untersuchung betrug die Mortalität $71 \%$ auf dem Hintergrund einer AKI-Prävalenz bei hämato-onkologischen Neoplasien (Soares et al. 2010). Die insgesamt unbefriedigenden Behandlungserfolge der gegenwärtigen Untersuchung müssen aber im Zusammenhang verstanden und auf den Umstand zurückgeführt werden, dass in der Zusammensetzung des Patientenkollektivs der nephrologischen ICU der UMG eine schwerpunktmässige Spezialisierung auf onkologische Nephropathien besteht.

\subsection{Maligne Neoplasien, Sepsis und AKI}

In Fortsetzung der Analyse wurde die Konstellation von Malignom, Sepsis und AKI untersucht. Die Prävalenz bei Komorbidität von Malignom, Sepsis und AKI war deutlich gegenüber der korrespondierenden Vergleichsgruppe ohne Sepsis erhöht (71,6\% vs. $32,2 \%$ ). Die Mortalität war bei analoger Betrachtung der beiden Vergleichsgruppen 
ebenfalls deutlich erhöht (67,6\% vs. 21,6\%). Mehr als $2 / 3$ der Patienten, die an einer malignen Neoplasie erkrankt waren und darüber hinaus eine Sepsis mit assoziiertem AKI entwickelten, waren im Zeitraum der Behandlungsdauer auf der ICU verstorben.

\subsection{Nierenersatztherapie bei Malignom und Sepsis}

Eine weitere Differenzierung der Untersuchung betraf die Komorbidität von malignen Neoplasien, Sepsis und dialysepflichtigem AKI, die eine besonders schwerwiegende Assoziation von schwersten Krankheitszuständen darstellt. Zu diesem Aspekt liegen in der Literatur keine vergleichbaren Untersuchungen vor. Die Prävalenz bei dialysepflichtigen Patienten und Vorliegen eines Malignoms ohne Sepsis betrug 51,0\%, bei Patienten mit Malignom und Sepsis 49,5\%. Danach hat die Koprävalenz einer Sepsis bei dialyspflichtigen Malignom-Patienten keinen Einfluß im Hinblick auf eine erhöhte Morbidität. Dagegen stellt die Koprävalenz der Sepsis bei Malignom-Patienten unter Dialysetherapie hinsichtlich der Mortalität einen dominierenden Faktor dar. Bei dialysepflichtigen Sepsis-Patienten ohne Malignom betrug die Mortalität 46,3\%, bei der weitaus ungünstigen Assoziation eines Malignoms 70,2\%. Auch die Mortalität wird vorrangig durch das Vorliegen einer Sepsis bestimmt, unabhängig vom gleichzeitigen Vorliegen einer malignen Tumorerkrankung als weiterer Komplikation.

Bei Erweiterung der Betrachtung mit Stratifikation zwischen soliden Tumoren und Hämoblastosen ergaben sich uneinheitliche Konstellationen des Behandlungserfolgs. Bei Koexistenz von Sepsis, dialysepflichtigen AKI und hämoblastischer Grunderkrankung, betrug die Mortalität 90,5\% und überstieg die mit 52,9\% geringere Mortalität der Patienten mit solider Tumorerkrankung. Bei den Patienten mit hämoblastischen Erkrankungen bedeutete das Erfordernis einer Nierenersatztherapie einen Anstieg der Mortalität um $33,3 \%$, von $64,3 \%$ auf $90,5 \%$. Hierbei ist allerdings zu betonen, dass die Nierenersatztherapie nicht als Ursache anzunehmen ist, vielmehr ist die Zunahme der Mortalität durch die Grunderkrankung trotz Nierenersatztherapie bedingt. Dagegen hatte der Einsatz der Dialysetherapie bei Koexistenz von Sepsis, dialysepflichtigen AKI und soliden Tumoren keinen erkennbaren Einfluß auf die ohnehin hohe Mortalität (52, 9\% vs. 56, 3\%). Eine einfache Erklärung für diese Unterschiede drängt sich nicht auf, naheliegend ist die Annahme unterschiedlicher pathophysiologischer Mechanismen und/oder unterschiedlicher kollateraler Effekte bei Einsatz aggessiver therapeutischer Behandlungsstrategien. 


\subsection{Leberzirrhose und AKI}

Bei Patienten mit chronischen Lebererkrankungen, insbesondere Leberzirrhose, stellt das AKI häufig eine lebensbedrohliche Komplikation mit ungünstiger Prognose dar (Rognant 2015). Die Inzidenz des AKI bei Patienten mit Leberzirrhose wird mit 20\% angegeben (Garcia-Tsao 2008; Wong 2013). Bei der Ätiologie des AKI bei chronischen Lebererkrankungen stehen prärenale hämodynamische Ursachen im Vordergrund wobei auch tubulus-schädigende Effekte erhöhter Bilirubin-Konzentrationen eine Rolle spielen (Davenport 2012; van Slambrouck 2013). Die Angaben zur Mortalität umfassen einen weiten Bereich von 34\% - 91\% (Cholongitas et al. 2009; Belcher et al. 2013; Wong et al. 2013). Die Ergebnisse der vorliegenden Untersuchung ergaben für Patienten mit Leberzirrhose und assoziiertem AKI vergleichsweise günstigere Werte mit einer Prävalenz von 8,2\% und einer Mortalität von 36,1\%. Als besondere Risikofaktoren konnten das Vorliegen einer Sepsis und die Notwendigkeit einer Nierenersatztherapie identifiziert werden. 


\section{Kapitel 5}

\section{Zusammenfassung und Perspektiven}

In dieser Untersuchung wurden die Behandlungsergebnisse einer hoch spezialisierten universitären nephrologischen Intensivstation über einen zurückliegenden Behandlungszeitraum von 3 Jahren analysiert. Die Schwerpunkte der Analyse betrafen das akute Nierenversagen in Zusammenhang mit den Komorbiditäten von Sepsis und malignen Neoplasien. Ungeachtet des Einsatzes von gegenwärtig modernsten Therapieverfahren ist die Mortalität bei Patienten mit AKI hoch $(\sim 40 \%)$. Bei Koinzidenz des AKI mit einer Sepsis war ein Anstieg der Mortalität zu verzeichnen $(\sim 52 \%)$. Bei Komorbidität von AKI und Hämoblastosen war eine weitere Steigerung der Mortalität erkennbar ( 60\%). Eine hinzukommende Sepsis führte zum Tod von mehr als 2/3 der Patienten. Die ungünstigte Komstellation ergab sich bei der Assoziation von Hämoblastom, Sepsis und dialysepflichtigem AKI mit einer extremen Mortalitätsziffer ( 90\%).

Insgesamt müssen die Behandlungserfolge im Hinblick auf die hohe Mortalität eher als unbefriedigend angesehen werden. Die ungünstige Bewertung des Behandlungserfolgs und die Prognose über den weiteren Krankheitsverlauf entziehen sich jedoch einfachen Beurteilungsmustern angesichts des hohen medizinischen Einsatzes und werden diesem nicht gerecht. Vielmehr weisen die Behandlungserfolge auf die Grenzen der modernen Intensivmedizin hin. Die Behandlungsergebnisse stehen in hohem Maße mit der demographischen Entwicklung der Bevölkerung in Zusammenhang, wonach die Inzidenz und eine veränderte Typologie schwerer Organerkrankungen mit Beginn der 6. Dekade deutlich zunehmen und Patienten auf den Intensivstationen sich häufig in multidimensionalen Krankheitszuständen befinden. 
Im Hinblick auf die Behandlungserfolge des akuten Nierenversagens sollte die Frage einer verbesserten Frühdiagnose durch den Einsatz von Biomarkern weiter wissenschaftlich vertieft werden. Die Bedeutung einer verbesserten Frühdiagnose in Bezug auf den Behandlungserfolg ist zur Zeit noch nicht abschließend geklärt. Ein verbessertes Verständnis der Pathophysiologie des akuten Nierenversagens insbesondere bei begleitenden Komorbiditäten von Sepsis und malignen Neoplasien sollte ultimativ zur Entwicklung verbesserter spezifischer Therapieformen und nierenprotektiver Verfahren führen. 


\section{Literaturverzeichnis}

1. Abercrombie J (1821): Observations on ischuria renalis. Edinburgh Med J 10, 210222

2. Ahmed W, Memon JI, Rehmani R, Al Juhaiman A (2014): Outcome of patients with acute kidney injury in severe sepsis and septic shock treated with early goaldirected therapy in an intensive care unit. Saudi J Kidney Dis Transpl 로, 544-551

3. Alkandari O, Eddington KA, Hyder A, Gauvin F, Ducruet T, Gottesman R, Phan V, Zappitelli M (2011): Acute kidney injury is an independent risk factor for pediatric intensive care unit mortality, longer length of stay and prolonged mechanical ventilation in critically ill children: a two-center retrospective cohort study. Crit Care 15, R146-R158

4. Alobaidi R, Basu RK, Goldstein SL, Bagshaw SM (2015): Sepsis-Associated Acute Kidney Injury. Semin Nephrol. $\underline{35}, 2-11$

5. Bagshaw SM, Uchino S, Bellomo R, Morimatsu H, Morgera S, Schetz M, Tan I, Bouman C, Macedo E, Gibney N et al. (2007): Septic acute kidney injury in critically ill patients: clinical characteristics and outcomes. Clin J Am Soc Nephrol $\underline{2}, 431-439$

6. Bagshaw SM, George C, Bellomo R (2008a): A comparison of the RIFLE and AKIN criteria for acute kidney injury in critically ill patients. Nephrol Dial Transplant 23, $1569-1574$

7. Bagshaw SM, George C, Bellomo R (2008b): Early acute kidney injury and sepsis: a multicentre evaluation. Crit Car 12, R47-R56

8. Bagshaw SM, Berthiaume LR, Delaney A, Bellomo R (2008c): Continuous versus intermittent renal replacement therapy for critically ill patients with acute kidney injury: a meta-analysis. Crit Care Med $\underline{36}, 610-617$

9. Bagshaw SM, Lapinsky S, Dial S, Arabi Y, Dodek P, Wood G, Ellis P, Guzman J, Marshall J, Parrillo JE et al. (2009): Acute kidney injury in septic shock: clinical outcomes and impact of duration of hypotension prior to initiation of antimicrobial therapy. Intensive Care Med $\underline{35}, 871-881$ 
10. Basu RK, Wong HR, Krawczeski CD, Wheeler DS, Manning PB, Chawla LS, Devarajan P, Goldstein SL (2014): Combining functional and tubular damage biomarkers improves diagnostic precision for acute kidney injury after cardiac surgery. J Am Coll Cardiol $\underline{64}, 2753-2762$

11. Belcher JM, Parikh CR, Garcia-Tsao G (2013): Acute kidney injury in patients with cirrhosis: perils and promise. Clin Gastroenterol Hepatol 11, 1550-1558

12. Bellomo R, Ronco C, Kellum JA, Mehta RL, Palevsky P (2004): Acute renal failure - definition, outcome measures, animal models, fluid therapy and information therapy needs: the Second International Consensus Conference of the Acute Dialysis Quality Initiative (ADQI) Group. Crit Care $\underline{8}$, R204-R212

13. Bellomo R, Cass A, Cole L, Finfer S, Gallagher M, Lo S, McArthur C, McGuinness S, Myburgh J, Norton R, et al. (2009): Intensity of continuous renal-replacement therapy in critically ill patients. N Engl J Med $\underline{361}$, 1627-1638

14. Bone RC, Balk RA, Cerra FB, Dellinger RP, Fein AM, Knaus WA, Schein RM, Sibbald WJ (1992): Definitions for sepsis and organ failure and guidelines for the use of innovative therapies in sepsis. The ACCP/SCCM Consensus Conference Committee. American College of Chest Physicians/Society of Critical Care Medicine. Chest $\underline{101}, 1644-1655$

15. Bougle A, Duranteau J (2011): Pathophysiology of sepsis-induced acute kidney injury: the role of global renal blood flow and renal vascular resistance. Contrib Nephrol 174, 89-97

16. Brivet FG, Kleinknecht DJ, Loirat P, Landais PJ (1996): Acute renal failure in intensive care units - causes, outcome, and prognostic factors of hospital mortality: A prospective, multicenter study. French Study Group on Acute Renal Failure. Crit Care Med 24, 192-198

17. Bywaters EG, Beall D (1941): Crush injuries with impairment of renal function. Br Med J 1, 427-432

18. Cartin-Ceba R, Kashiouris M, Plataki M, Kor DJ, Gajic O, Casey ET (2012): Risk factors for development of acute kidney injury in critically ill patients: a systematic review and meta-analysis of observational studies. Crit Care Res Pract 2012:691013

19. Cholongitas E, Calvaruso V, Senzolo M, Patch D, Shaw S, O'Beirne J, Burroughs AK (2009): RIFLE classification as predictive factor of mortality in patients with cirrhosis admitted to intensive care unit. J Gastroenterol Hepatol 24, 1639-1647 
20. Christiansen CF, Johansen MB, Langeberg WJ, Fryzek JP, Sorensen HT (2011): Incidence of acute kidney injury in cancer patients: a Danish population-based cohort study. Eur J Intern Med $\underline{4}, 399-406$

21. Choudhury D, Ahmed Z (2006): Drug-associated renal dysfunction and injury. Nat Clin Pract Nephrol 2, 80-91

22. Coca SG, Garg AX, Thiessen-Philbrook H, Koyner JL, Patel UD, Krumholz HM, Shlipak MG, Parikh CR (2014): Urinary biomarkers of AKI and mortality 3 years after cardiac surgery. J Am Soc Nephrol $\underline{25}$, 1063-1071

23. Cruz DN, Bolgan I, Perazella MA, Bonello M, de Cal M, Corradi V, Polanco N, Ocampo C, Nalesso F, Piccinni P et al. (2014): North East Italian Prospective Hospital Renal Outcome Survey on Acute Kidney Injury (NEiPHROS-AKI): targeting the problem with the RIFLE Criteria. Clin J Am Soc Nephrol 2 , 418-425

24. Daher EF, Marques CN, Lima RS, Silva Junior GB, Barbosa AS, Barbosa ES, Mota RM, Leite da Silva S, Araujo SM, Liborio AB (2008): Acute kidney injury in an infectious disease intensive care unit - an assessment of prognostic factors. Swiss Med Wkly 138, 128-133

25. Darmon M, Thiery G, Ciroldi M, Porcher R, Schlemmer B, Azoulay E (2007): Should dialysis be offered to cancer patients with acute kidney injury? Intensive Care Med 33, 765-772

26. Davenport A (2012): AKI in a patient with cirrhosis and ascites. Clin J Am Soc Nephrol 7, 2041-2048

27. Davies FC, Weldon RP (1917): A contribution to the study of war nephritis. Lancet $\underline{2}, 118-120$

28. Eberle J: A Treatise on the Practice of Medicine. Band 2, 5. Auflage; Gregg \& Elliot, Philadelphia 1841, 402-406

29. Fife G (1840): Provincial Medical Association - Poor-Law Report. Prov Med Surg $\mathrm{J} \underline{1}, 211-212$

30. Garcia-Tsao G, Parikh CR, Viola A (2008): Acute kidney injury in cirrhosis. Hepatology $\underline{48}, 2064-2077$

31. Graefe E: Ischuria. In: Busch DWH, v. Gräfe CF, Horn E, Link HF, Müller J, Osann E (Hrsg.): Enzyklopädisches Wörterbuch der medicinischen Wissenschaften. Band 19, Verlag von Heit et Comp., Berlin 1839, 231-245 
32. Guerin C, Girard R, Selli JM, Perdrix JP, Ayzac L (2000): Initial versus delayed acute renal failure in the intensive care unit: A multicenter prospective epidemiological study. Rhone-Alpes Area Study Group on Acute Renal Failure. Am J Respir Crit Care Med 161, 872-879

33. Harris DG, McCrone MP, Koo G, Weltz AS, Chiu WC, Scalea TM, Diaz JJ, Lissauer ME (2015): Epidemiology and outcomes of acute kidney injury in critically ill surgical patients. J Crit Care $\underline{30}, 102-106$

34. Hoste EA, Lameire NH, Vanholder RC, Benoit DD, Decruyenaere JM, Colardyn FA (2003): Acute renal failure in patients with sepsis in a surgical ICU: predictive factors, incidence, comorbidity, and outcome. J Am Soc Nephrol 14, 1022-1030

35. Hoste EA, Kellum JA (2006): Acute kidney injury: epidemiology and diagnostic criteria. Curr Opin Crit Care $\underline{6}, 531-537$

36. Hoste EAJ, Clermont G, Kersten A, Venkataraman R, Angus DC, De Bacquer D, Kellum JA (2006): RIFLE criteria for acute kidney injury are associated with hospital mortality in critically ill patients: a cohort analysis. Crit Care 10, R73-R82

37. Hou SH, Bushinsky DA, Wish JB, Cohen JJ, Harrington JT (1983): Hospitalacquired renal insufficiency: A prospective study. Am J Med 4ㅗ, 243-248

38. Humphreys BD: Renal Complications of Hematopoietic Stem Cell Transplantation. In: Cancer and the Kidney. 2. Auflage; Elsevier Press, London 2011, 205-220

39. Karlsson S, Varpula M, Ruokonen E, V. Pettilä V, Parviainen I, Ala-Kokko TI, Kolho E, Rintala EM (2007): Incidence, treatment, and outcome of severe sepsis in ICU-treated adults in Finland: the Finnsepsis study. Intensive Care Med $\underline{33}$, $435-443$

40. Karvellas CJ, Farhat MR, Sajjad I, Mogensen SS, Leung AA, Wald R, Bagshaw SM (2011): A comparison of early versus late initiation of renal replacement therapy in critically ill patients with acute kidney injury: a systematic review and metaanalysis. Crit Care 15, R72-R82

41. KDIGO (2012): Kidney Disease: Improving Global Outcomes (KDIGO) Acute Kidney Injury Work Group. KDIGO Clinical Practice Guideline for Acute Kidney Injury. Kidney Inter, Suppl. $\underline{2}, 1-138$

42. Kellum JA (2011): Impaired renal blood flow and the 'spicy food' hypothesis of acute kidney injury. Crit Care Med 39, 901-903

43. Kellum JA, Levin N, Bouman C, Lameire N (2002): Developing a consensus classification system for acute renal failure. Curr Opin Crit Care $\underline{8}, 509-514$ 
44. Kellum JA, Mythen MG, Shaw AD (2014): The 12th consensus conference of the Acute Dialysis Quality Initiative (ADQI XII). Br J Anaesth 113, 729-731

45. Lahoti A, Kantarjian H, Salahudeen AK, Ravandi F, Cortes JE, Faderl S, O’Brien S, Wierda W, Mattiuzzi GN (2010): Predictors and outcome of acute kidney injury in patients with acute myelogenous leukemia or high-risk myelodysplastic syndrome. Cancer 116, 4063-4068

46. Lam AQ, Humphreys BD (2012): Onco-nephrology: AKI in the cancer patient. Clin J Am Soc Nephrol ㅁ, 1692-1700

47. Lameire N (2014): Nephrotoxicity of recent anti-cancer agents. Clin Kidney J $\underline{7}$, $11-22$

48. Le Gall JR, Lemeshow S, Saulnier F (1993): A new Simplified Acute Physiology Score (SAPS II) based on a European/North American multicenter study. JAMA $\underline{270}, 2957-2963$

49. Levy EM, Viscoli CM, Horwitz RI (1996): The effect of acute renal failure on mortality. A cohort analysis. JAMA $\underline{275}, 1489-1494$

50. Levy MM, Fink MP, Marshall JC, Abraham E, Angus D, Cook D, Cohen J, Opal SM, Vincent JL, Ramsay G (2003): 2001 SCCM/ESICM/ACCP/ATS/SIS International Sepsis Definitions Conference. Crit Care Med 31, 1250-1256

51. Liano F, Pascual J (1996): Epidemiology of acute renal failure: A prospective, multicenter, community-based study. Madrid Acute Renal Failure Study Group. Kidney Int $\underline{50}, 811-818$

52. Liano F, Junco E, Pascual J, Madero R, Verde E (1998): The spectrum of acute renal failure in the intensive care unit compared with that seen in other settings: the Madrid Acute Renal Failure Study Group. Kidney Int Suppl 66, S16-S24

53. Liu KD, Thompson BT, Ancukiewicz M, Steingrub JS, Douglas IS, Matthay MA, Wright P, Peterson MW, Rock P, Hyzy RC et al. (2011): Acute kidney injury in patients with acute lung injury: impact of fluid accumulation on classification of acute kidney injury and associated outcomes. Crit Care Med $\underline{39}$, 2665-2671

54. Lopes JA, Jorge S, Resina C, Santos C, Pereira A, Neves J, Antunes F, Prata MM (2007): Acute renal failure in patients with sepsis. Crit Care 11, 411-412

55. Lopes JA, Jorge S (2011): Acute kidney injury following HCT: incidence, risk factors and outcome. Bone Marrow Transplant 뜨, 1399-1408 
56. Lopes JA, Jorge S, Resina C, Santos C, Pereirab A, Neves J, Antunes F, Prata MM (2009): Acute kidney injury in patients with sepsis: a contemporary analysis: Int J Infec Dis $\underline{13}, 176-181$

57. Maccariello E, Valente C, Nogueira L, Bonomo H Jr, Ismael M, Machado JE, Baldotto F, Godinho M, Rocha E, Soares M (2011): Outcomes of cancer and noncancer patients with acute kidney injury and need of renal replacement therapy admitted to general intensive care units. Nephrol Dial Transplant 26, 537-543

58. McCullough PA, Bouchard J, Waikar SS, Siew ED, Endre ZH, Goldstein SL, Koyner JL, Macedo E, Doi K, Di Somma S et al. (2013): Implementation of novel biomarkers in the diagnosis, prognosis, and management of acute kidney injury: executive summary from the tenth consensus conference of the Acute Dialysis Quality Initiative (ADQI). Contrib Nephrol 182, 5-12

59. Mehta RL, Pascual MT, Soroko S, Savage BR, Himmelfarb J, Ikizler TA, Paganini EP, Chertow GM (2004): Spectrum of acute renal failure in the intensive care unit: The PICARD experience. Kidney Int $\underline{66}, 1613-1621$

60. Mehta RL, Kellum JA, Shah SV, Molitoris BA, Ronco C, Warnock DG, Levin A (2007): Acute Kidney Injury Network: report of an initiative to improve outcomes in acute kidney injury. Crit Care $\underline{11}, \mathrm{R} 31-\mathrm{R} 39$

61. Murugan R, Kellum JA (2011): Acute kidney injury: what's the prognosis? Nat Rev Nephrol 7, 209-217

62. Nash K, Hafeez A, Hou S (2002): Hospital-acquired renal insufficiency. Am J Kidney Dis $\underline{39}, 930-936$

63. Neveu H, Kleinknecht D, Brivet F, Loirat P, Landais P (1996): Prognostic factors in acute renal failure due to sepsis. Results of a prospective multicentre study. The French Study Group on Acute Renal Failure. Nephrol Dial Transplant 11, 293-299

64. Nisula S, Kaukonen KM, Vaara ST, Korhonen AM, Poukkanen M, Karlsson S, Haapio M, Inkinen O, Parviainen I, Suojaranta-Ylinen R et al. (2013): Incidence, risk factors and 90-day mortality of patients with acute kidney injury in Finnish intensive care units: the FINNAKI study. Intensive Care Med 39, 420-428

65. Oppert M, Engel C, Brunkhorst FM, Bogatsch H, Reinhart K, Frei U, Eckardt KU, Loeffler M, John S (2008): Acute renal failure in patients with severe sepsis and septic shock a significant independent risk factor for mortality: results from the German Prevalence Study. Nephrol Dial Transplant 23, 904-909 
66. Osler W: The Principles and Practice of Medicine. 8. Auflage; Butterworth \& Co. (India) Ltd., Calcutta 1912

67. Ostermann M, Chang RW (2007): Acute kidney injury in the intensive care unit according to RIFLE. Crit Care Med $\underline{35}$, 1837-1843

68. Palevsky PM, Zhang JH, O'Connor TZ, Chertow GM, Crowley ST, Choudhury D, Finkel K, Kellum JA, Paganini E, Schein RM et al. (2008): Intensity of renal support in critically ill patients with acute kidney injury. N Engl J Med 359, 7-20

69. Plataki M, Kashani K, Cabello-Garza J, Maldonado F, Kashyap R, Kor DJ, Gajic O, Cartin-Ceba R (2011): Predictors of acute kidney injury in septic shock patients: an observational cohort study. Clin J Am Soc Nephrol $\underline{6}, 1744-1751$

70. Porter CJ, Juurlink I, Bisset LH, Bavakunji R, Mehta RL, Devonald MA (2014): A real-time electronic alert to improve detection of acute kidney injury in a large teaching hospital. Nephrol Dial Transplant 299, 1888-1893

71. Rangel-Frausto MS, Pittet D, Costigan M, Hwang T, Davis CS, Wenzel RP (1995): The natural history of the systemic inflammatory response syndrome (SIRS). A prospective study. JAMA $\underline{273}, 117-123$

72. Ratanarat R, Skulratanasak P, Tangkawattanakul N, Hantaweepant C (2013): Clinical accuracy of RIFLE and Acute Kidney Injury Network (AKIN) criteria for predicting hospital mortality in critically ill patients with multi-organ dysfunction syndrome. J Med Assoc Thai 96 Suppl 2, S224-S231

73. Rewa O, Bagshaw SM (2014): Acute kidney injury - epidemiology, outcomes and economics. Nat Rev Nephrol 10, 193-207

74. Ricci Z, Ronco C (2009): Pathogenesis of acute kidney injury during sepsis. Curr Drug Targets $\underline{12}, 1179-1183$

75. Rognant N (2015): Acute kidney injury in patients with chronic liver disease. World J Hepatol 포 993-1000

76. Ronco C, Bellomo R, Homel P, Brendolan A, Dan M, Piccinni P, La Greca G (2000): Effects of different doses in continuous veno-venous haemofiltration on outcomes of acute renal failure: a prospective randomised trial. Lancet $\underline{356}, 26-30$

77. Salahudeen AK, Kumar V, Madan N, Xiao L, Lahoti A, Samuels J, Nates J, Price K (2009): Sustained low efficiency dialysis in the continuous mode (C-SLED): dialysis efficacy, clinical outcomes, and survival predictors in critically ill cancer patients. Clin J Am Soc Nephrol 4, 1338-1346 
78. Salahudeen AK, Doshi SM, Pawar T, Nowshad G, Lahoti A, Shah P (2013): Incidence rate, clinical correlates, and outcomes of AKI in patients admitted to a comprehensive cancer center. Clin J Am Soc Nephrol ㅁ, 347-354

79. Schrier RW, Wang W, Poole B, Mitra A (2004): Acute renal failure: definitions, diagnosis, pathogenesis, and therapy. J Clin Invest 114, 5-14

80. Schwarz A: Systematik der Nephrotoxizität. In: Nierenfunktion und Arzneimittel. Deuber HJ, Keller F, Schwarz A (Hrsg.): Wissenschaftliche Verlagsgesellschaft, Stuttgart 2001, 150-259

81. Shusterman N, Strom BL, Murray TG, Morrison G, West SL, Maislin G (1987): Risk factors and outcome of hospital-acquired acute renal failure: Clinical epidemiologic study. Am J Med $\underline{83}, 65-71$

82. Siew ED, Davenport A (2015): The growth of acute kidney injury: a rising tide or just closer attention to detail? Kidney Int $\underline{87}, 46-61$

83. Siew ED, Matheny ME, Ikizler TA, Lewis JB, Miller RA, Waitman LR, Go AS, Parikh CR, Peterson JF (2010): Commonly used surrogates for baseline renal function affect the classification and prognosis of acute kidney injury. Kidney Int $\underline{77}$, 536-542

84. Singbartl K, Kellum JA (2012): AKI in the ICU: definition, epidemiology, risk stratification, and outcomes. Kidney Int $\underline{81}, 819-825$

85. Smith HW: The Kidney: Structure and Function in Health and Disease. Oxford University Press, New York 1951

86. Soares M, Lobo SMA, Torelly AP, de Carvalho Mello PV, Silva U, Meira Teles JM, Silva E, Caruso P, Friedman G, Pereira de Souza PC et al. (2010): Outcomes of cancer patients admitted to Brazilian intensive care units with severe acute kidney injury. Rev bras ter intensiva $\underline{22}, 236-244$

87. Sood MM, Shafer LA, Ho J, Reslerova M, Martinka G, Keenan S, Dial S, Wood G, Rigatto C, Kumar A (2014): Early reversible acute kidney injury is associated with improved survival in septic shock. J Crit Care 29, 711-717

88. Susantitaphong P, Cruz DN, Cerda J, Abulfaraj M, Alqahtani F, Koulouridis I, Jaber BL (2013): World incidence of AKI: a meta-analysis. Clin J Am Soc Nephrol $\underline{8}, 1482-1493$

89. Sutherland SM, Zappitelli M, Alexander SR, Chua AN, Brophy PD, Bunchman TE, Hackbarth R, Somers MJ, Baum M, Symons JM et al. (2010): Fluid overload and mortality in children receiving continuous renal replacement therapy: the 
prospective pediatric continuous renal replacement therapy registry. Am J Kidney Dis $\underline{55}, 316-325$

90. Teasdale G, Jennett B (1974): Assessment of coma and impaired consciousness. A practical scale. Lancet $\underline{2}, 81-84$

91. Uchino S, Bellomo R, Morimatsu H, Morgera S, Schetz M, Tan I, Bouman C, Macedo E, Gibney N, Tolwani A et al. (2005a): External validation of severity scoring systems for acute renal failure using a multinational database. Crit Care Med 33, 1961-1967

92. Uchino S, Kellum JA, Bellomo R, Doig GS, Morimatsu H, Morgera S, Schetz M, Tan I, Bouman C, Macedo E et al. (2005b): Acute renal failure in critically ill patients: A multinational, multicenter study. JAMA 294, 813-818

93. van Slambrouck CM, Salem F, Meehan SM, Chang A (2013): Bile cast nephropathy is a common pathologic finding for kidney injury associated with severe liver dysfunction. Kidney Int $\underline{84}, 192-197$

94. Waikar SS, Curhan GC, Wald R, McCarthy EP, Chertow GM (2006): Declining mortality in patients with acute renal failure, 1988 to 2002. J Am Soc Nephrol 17, $1143-1150$

95. Waikar SS, Liu KD, Chertow GM (2008): Diagnosis, Epidemiology and Outcomes of Acute Kidney Injury. Clin J Am Soc Nephrol $\underline{3}$, 844-861

96. Wan L, Bagshaw SM, Langenberg C, Saotome T, May C, Bellomo R (2008): Pathophysiology of septic acute kidney injury: what do we really know? Crit Care Med $\underline{36}$ (Suppl), S198-S203

97. Wong F (2013): Acute renal dysfunction in liver cirrhosis. Gastroenterol Hepatol $\underline{9}, 830-832$

98. Wong F, O'Leary JG, Reddy KR, Patton H, Kamath PS, Fallon MB, GarciaTsao G, Subramanian RM, Malik R, Maliakkal B et al. (2013): New consensus definition of acute kidney injury accurately predicts 30-day mortality in patients with cirrhosis and infection. Gastroenterology $\underline{145}, 1280-1288$

99. Xue JL, Daniels F, Star RA, Kimmel PL, Eggers PW, Molitoris BA, Himmelfarb J, Collins AJ (2006): Incidence and mortality of acute renal failure in Medicare beneficiaries, 1992 to 2001. J Am Soc Nephrol 17, 1135-1142

100. Yegenaga I, Hoste E, Van Biesen W, Vanholder R, Benoit D, Kantarci G, Dhondt A, Colar-dyn F, Lameire N (2004): Clinical characteristics of patients developing 
ARF due to sepsis/systemic inflammatory response syndrome: results of a prospective study. Am J Kidney Dis $\underline{43}, 817-824$

101. Ympa YP, Sakr Y, Reinhart K, Vincent JL (2005): Has mortality from acute renal failure decreased? A systematic review of the literature. Am J Med 118, 827-32

102. Yorkes W, Nauss RN (1911): The mechanism of the production of suppression of urine in blackwater fever. Ann Trop Med Parasitol 12, 287-312

103. Zarbock A, Gomez H, Kellum JA (2014): Sepsis-induced acute kidney injury revisited: pathophysiology, prevention and future therapies. Curr Opin Crit Care $\underline{6}$, $588-595$

104. Zarjou A, Agarwal A (2011): Sepsis and Acute Kidney Injury. J Am Soc Nephrol 22, 999-1006 


\section{Anhang}




\title{
Prognosis of $\mathrm{AKI}$ in malignant diseases with and without sepsis
}

\author{
Malte Heeg ${ }^{1}$, Alexander Mertens ${ }^{1}$, David Ellenberger ${ }^{2}$, Gerhard A Müller ${ }^{1}$ and Daniel Patschan ${ }^{1 *}$
}

\begin{abstract}
Background: AKI significantly worsens prognosis of hospitalized patients. This is particularly the case in patients with sepsis. The risk for aquiring sepsis is significantly increased in malignant diseases. Aim of the present retrospective study was to analyze outcomes of tumor patients with sepsis and AKI.

Methods: One-thousand and seventeen patients, treated at the ICU of the Department of Nephrology and Rheumatology of the University Hospital Göttingen from 2009 to 2011 were retrospectively analyzed for mortality, sepsis, AKI, need for renal replacement therapy (dialysis) and malignancies.

Results: AKI occurred significantly more frequent in septic than in non-septic patients and in tumor as oposed to non-tumor patients. Mortaliy rates were higher in the respective latter groups. Mortality increased even further if patients suffered from a malignant disease with sepsis and AKI. Mortality rates peaked if dialysis treatment became mandatory. In non-solid tumors $100 \%$ of the patients died if they suffered drom sepsis and AKI. This was not the case in solid malignancies (mortality rate 56\%).
\end{abstract}

Conclusions: We conclude that prognosis of tumor patients with AKI and sepsis is very poor. Mortality increases to almost $70 \%$ if diaylsis therapy is initiated. Non-solid tumors are associated with a 100\% mortality if sepsis and AKI conincide.

Keywords: AKl, Sepsis, Malignancies, Mortality

\section{Background}

Acute kidney injury is one of the major problems in today's clinical medicine. Approximately 1-5\% of all hospitalized patients develop AKI during the course of the treatment $[1,2]$. Prognosis has not substantially been improved during the last $20-30$ years since mortality rates still vary between $30-50 \%$ [3]. The poor prognosis is not exclusively induced by AKI per se but does also result from the underlying causes / diseases leading to the decline in kidney function [4]. This is particularly the case with conditions that compromize oxygen and nutrient supply of the whole organism. Among those are heart failure and sepsis. Especially the latter has been identified as one of the most potent risk factors for AKI during intensive care treatment [5,6]. According to newer data, about $50 \%$ of all sepsis patients treated at the ICU suffer from AKI of various severity. The average

* Correspondence: d.patschan@gmail.com

'Department of Nephrology and Rheumatology, University Hospital of

Göttingen, Robert-Koch-Straße 40, 37075 Göttingen, Germany

Full list of author information is available at the end of the article mortality in sepsis-associated AKI is $50 \%$, even if dialysis treatment has been initiated [7]. The risk for sepsis is being increased by circumstances that affect the immunological response of the host. This typically occurs in malignant diseases. The risk for infectious complications in tumor patients is dramatically increased by bone marrow-toxic chemotherapeutics. Cytotoxic treatment on the other hand can also induce AKI, depending on the type of drugs used for therapy. Maccariello and colleagues analyzed the outcome of ICU patients requiring renal replacement therapy in a prospective manner [8]. The study failed to show an association between mortality and cancer. Nevertheless, mortality was higher if patients suffered from sepsis. The authors did not separately analyze sepsis patients with a malignant disease.

Aim of the this retrospective observational single center study was to analyze outcomes of ICU patients with AKI. Thereby, our particular interest focused on mortality of AKI in malignancies with versus without sepsis. 


\section{Methods \\ Patients and setting}

The present investigation was a retrospective singlecentre analysis. All patients treated at the medical intensive care unit of the department of nephrology and rheumatology (University Hospital of Göttingen, Germany) between 2009 and 2011 were included into the study. It was formally approved by the local ethics committee. Acute kidney injury was defined using the AKIN criteria [9]. Patients with pre-existing ESRD (end stage renal disease) were also included into the study. In these patients, any further acute aggravation of renal dysfunction was defined as AKI if the AKIN criteria were applicable and / or if, for other reasons, dialysis treatment was initiated. Indications for dialysis were the presence of one or more of the following criteria: refractory hyperkalemia, increases of serum creatinine $>3$ $\mathrm{mg} / \mathrm{dl}$ and / or of blood urea nitrogen $>100 \mathrm{mg} / \mathrm{dl}$ at any given time point, and signs / symptoms of fluid overload due to dimished urine output, respectively. As in earlier studies [10], sepsis was defined as systemic inflammatory response syndrome (SIRS) of infectious origin [11]. Thus, beside fulfilling the criteria of SIRS [12], all patients showed at least once positive blood cultures for either Gram-positive or Gram-negative bacteria and/or clinical symptoms of an infectous disease. The term malignancy was used in any case of tumor manifestation at the time of treatment at the ICU, regardless of the respective stage of the disease. Thereby, we differentiated between solid and non-solid (hemato-oncological) malignancies. For further clinical characterization a number of different parameters, such as c-reactive protein and the SAPS (Simplified Acute Physiology Score) II scores were documented on a daily basis. All data analyzed in this study were extracted from a database, belonging to the department of Nephrology \& Rheumatology of the University Hospital of Göttingen.

\section{Statistical analysis}

All results are expressed as percentages. Differences between 3 or more groups were analyzed by ANOVA. Differences between two groups were analyzed by chisquare test. Significance was considered at $\mathrm{p}<0.05$.

\section{Results}

\section{Patients}

A total of 1.017 patients were included into the study. Six-hundred and eight were male, 409 were female, the mean age of all patients was $65 \pm 16$ years with $65 \pm 14$ years in men and $66 \pm 18$ years in women. All patients were treated at the intensive care unit of the department of nephrology and rheumatology of the university hospital Göttingen (Germany) between 2009 and 2011. Sepsis was diagnosed in 330 patients (32\% - 208 male [63\%],
122 female [37\%]), 687 patients (68\%) did not fulfill the respective criteria. Two-hundred and twelve patients (21\% - 138 male [65\%], 74 female [35\%]) suffered from a malignant disease at the time of admission to the ICU (non-solid tumor: 88, solid tumor: 124). Thirty-three patients with a non-solid tumor underwent bone marrow-/stem cell transplantation in their history. Fourhundred and thirty-five patients (43\% - 278 male [64\%], 157 female [36\%]) either presented with AKI at the time of ICU admission or developed AKI during the treatment course at the ICU. Liver cirrhosis was diagnosed in 83 patients ( $8 \%$ - 57 male [69\%], 25 female [31\%]). The most important general outcome parameters of all included patients are summarized in Tables 1 and 2.

\section{AKI in sepsis}

As pointed out earlier sepsis was diagnosed in 330 patients (32\% - 208 male [63\%], 122 female [37\%]), 687 patients $(68 \%)$ did not fulfill the respective criteria. In the no sepsis group 474 patients (69\%) did not develop AKI while 213 (31\%) presented with AKI of various severity. One-hundred and eight patients (32\%) in the sepsis group did not suffer from AKI during the course of the disease, but AKI developed in 222 individuals (68\%). Thus, AKI occured significantly more frequent in sepsis than in non-septic patients $(\mathrm{p}<0.001)$. The mortality rates within the respective groups were: no sepsis without AKI - 15\% (72/474), no sepsis with AKI - 15\% (31/213), sepsis without AKI - 36\% (39/108), and sepsis with AKI - 53\% (117/222). Mortality rates were significantly higher in patients with sepsis $( \pm$ AKI) as compared to those without sepsis $( \pm \mathrm{AKI})$. Additionally,

\section{Table 1 Outcome characteristics}

\begin{tabular}{llll}
\hline Variables & Survivors & Non-survivors & p-value \\
\hline Age & $64.3 \pm 16.6$ years & $69.5 \pm 13.5$ years & $<0.001$ \\
Male gender & $440(58 \%)$ & $168(64 \%)$ & 0.066 \\
SAPS II & $30.4 \pm 10.7$ & $41.8 \pm 10.9$ & $<0.001$ \\
AKI & $287(37 \%)$ & $148(56 \%)$ & $<0.001$ \\
Sepsis & $175(23 \%)$ & $156(60 \%)$ & $<0.001$ \\
AKI+Sepsis & $105(16 \%)$ & $117(45 \%)$ & $<0.001$ \\
Cancer & $135(17 \%)$ & $76(29 \%)$ & $<0.001$ \\
Liver cirrhosis & $52(7 \%)$ & $30(12 \%)$ & 0.017 \\
Dialysis & $224(30 \%)$ & $120(46 \%)$ & $<0.001$ \\
ICU stay & $7.4 \pm 8.1$ days & $7.4 \pm 8.4$ days & 0.9 \\
Controlled ventilation & $244(32 \%)$ & $185(71 \%)$ & $<0.001$
\end{tabular}

Table 1 compares frequencies of different variables / mortality risk factors between survivors and non-survivors. The differences between the two groups were significant in all analyzed categories, with the exception of male gender and length of ICU stay. Table 1 shows frequencies of the three AKIN stages in AKI patients if used for diagnosis, followed by the respective mean serum AKI patients if used for diagnosis, followed by the respective mean serum
creatinine concentrations at the time of admission to the ICU (results as mean \pm SEM) 
Table 2 Serum creatinine concentrations in AKI patients before onset of AKI

\begin{tabular}{lll}
\hline AKIN stage & $\mathbf{n}$ & $\begin{array}{l}\text { [Creatinine] }]_{\mathbf{S}} \text { before } \\
\text { onset of AKI (mg/dl) }\end{array}$ \\
\hline 1 & 27 & $1.3 \pm 0.1$ \\
2 & 14 & $2.4 \pm 0.3$ \\
3 & 95 & $3.7 \pm 0.2$ \\
\hline
\end{tabular}

patients with sepsis and AKI died more frequently than those wihtout AKI (Figure 1).

\section{AKI in malignancies}

Two-hundred and twelve patients $(21 \%$ - 138 male [65\%], 74 female [35\%]) suffered from a malignant disease at the time of admission to the ICU. A solid tumor was diagnosed in 123 patients (58\%) while a non-solid tumor was apparent in 87 patients (42\%). Incidences of AKI were: patients without malignancy 329/805 (41\%), patients with a malignant disease (either solid or nonsolid tumor) 104/212 (49\%). The difference was statistically significant $(\mathrm{p}=0.032)$. Mortalty rates were: patients without tumor but with AKI 93/329 (28\%), patients with malignancy and AKI 53/104 (51\%). The calculated p-value was below 0.001 . Thus, the coincidence of a malignant disease and AKI dramatically worsened the overall prognosis (Figure 2).

\section{AKI in malignancies with sepsis}

One-hundred and fifteen patients with a malignant disease did not suffer from sepsis $(54 \%$ - 115/212). In this group, the incidence of AKI was 30\% (35/115) and mortality was $22 \%(8 / 35)$. Ninety-seven patients with a malignancy were diagnosed with sepsis (46\% - 97/212). In this particular group, AKI occurred in 69\% (67/97), mortality was $67 \%(45 / 67)$. In both categories (incidence of AKI, mortality) differences between the two groups (tumor with vs. without sepsis) were significant (p-values lower than 0.001 - Figure 3). In a subgroup analysis, mortality rates of patients with sepsis and AKI were compared, depending on the presence of a solid versus non-solid tumor disease. As a matter of fact, patients with a non-solid tumor died in 100\% if sepsis and AKI coincided, as compared to those with a solid tumor disease. In the latter group, mortality was $56 \%(\mathrm{p}<0.001)$.

\section{Mortality and dialysis}

A total number of 341 patients required dialysis treatment. Subgroup analysis revealed the following frequencies of renal replacement therapy in patients with versus without malignant diseases: patients without tumor and without sepsis (28\%) 94/341, mortality was 15\% (14/94), patients without tumor but with sepsis (28\%) 95/341, mortality was $46 \%$ (44/95), patients with tumor and without sepsis $6 \%$ (20/341), mortality was $20 \%(4 / 20)$, patients with tumor plus sepsis $11 \%(36 / 341)$, mortality was $66 \%(24 / 36)$. The following differences in mortality rates were statistically significant between the groups: no tumor, no sepsis versus no tumor, sepsis $\mathrm{p}<0.001$, tumor, no sepsis versus tumor, sepsis $\mathrm{p}<0.001$, no tumor, sepsis versus tumor, sepsis $\mathrm{p}=0.037$.

\section{Discussion}

Aim of this study was to retrospectively analyze epidemiology and outcome of ICU patients with AKI. Our

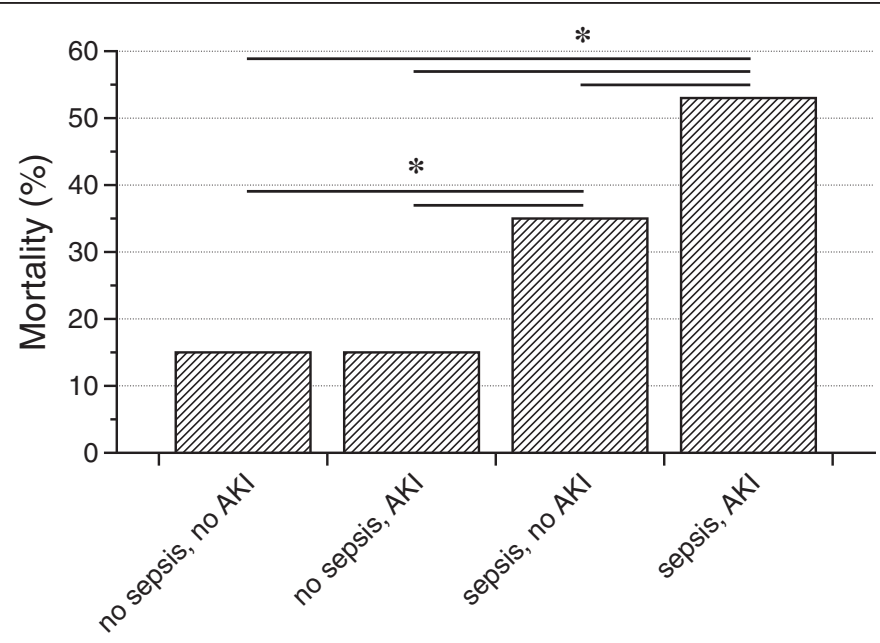

Figure 1 Mortality in septic versus non-septic patients with and without AKI. The prognosis did not differ between non-septic patients with or without AKI, mortality was $15 \%$ in both groups (no AKI $72 / 474$, AKI 31/213). Septic patients had higher mortality rates as oposed to those without sepsis, independently from the presence of AKI (sepsis without AKI - 36\% (39/108), sepsis with AKI - 53\% (117/222)) (*: p<0.001). 


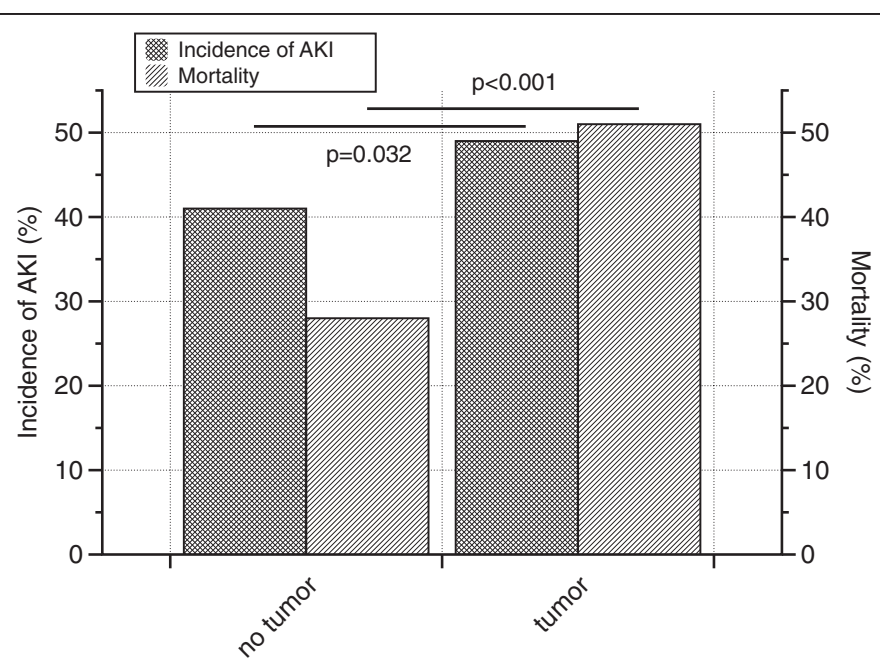

Figure 2 Incidence of AKI and mortality in tumor versus non-tumor patients. Incidences of AKI were: patients without malignancy 329/805 (41\%), patients with a malignant disease (either solid or non-solid tumor) 104/212 (49\%). The difference was statistically significant ( $p=0.032$ ). Mortalty rates were: patients without tumor but with AKI 93/329 (28\%), patients with malignancy and AKI 53/104 (51\%). The calculated p-value was below 0.001

particular interest focused on AKI mortality rates in malignancies and sepsis.

AKI significantly worsens the prognosis of hospitalized patients $[2,4]$. This is particularly the case in the ICU setting in which mortality can increase to 60\% [4]. Meanwhile, sepsis / septic shock have been emerged as the most frequent causes of AKI at the intensive care unit [6]. The pathogenesis of sepsis-associated AKI is complex and includes severe hemodynamic alterations with subsequent renal hypoperfusion on one hand, but also systemic activation of the innate and aquired immune response leading to inflammation of the kidney on the other hand $[6,13]$. The processes involved shall not be reviewed more in detail at the moment. However, regarding the widespread host-initiated defense mechanisms it becomes understandable that mortality rates gradually increase with progressive severity of the septic syndrome [14]. The prognosis of sepsis-associated AKI

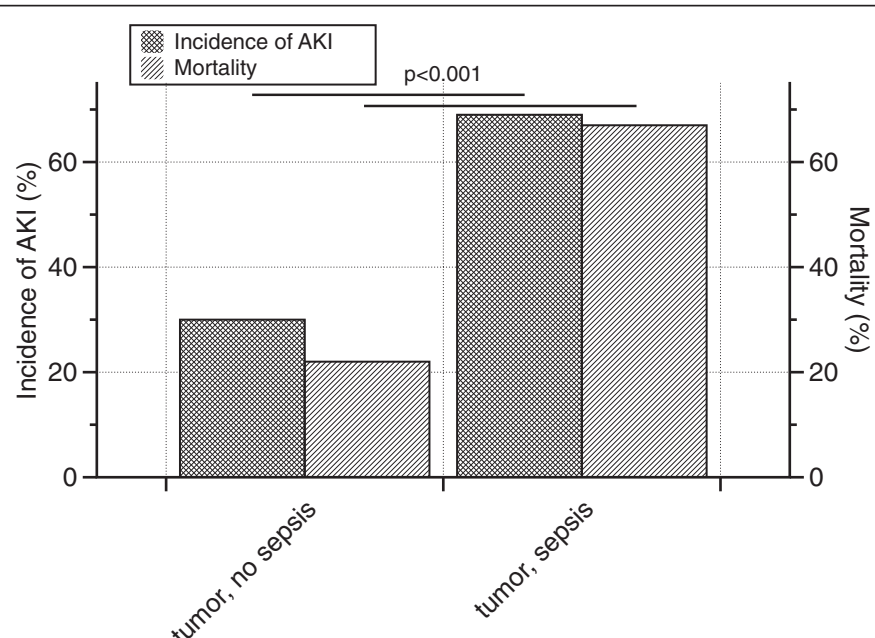

Figure 3 Incidence of AKI and mortality in tumor patients with versus without sepsis. One-hundred and fifteen patients with a malignant disease did not suffer from sepsis (54\% - 115/212). The incidence of AKI was 30\% (35/115) and mortality was 22\% (8/35). Ninety-seven patients with a malignancy were diagnosed with sepsis (46\% - 97/212). AKI occurred in 69\% (67/97), mortality was 67\% (45/67). 
also depends on the severity of renal dysfunction per se: Bagshaw and colleagues showed higher AKI mortality rates in septic shock with progressive deterioration of renal function [15]. Interestingely, the authors did not find higher incidences of AKI if the diagnosis of metastatic solid organ cancer was made [15]. Patients with non-solid hematologic malignacies on the other hand developed AKI more frequently. Finally, Plataki and colleagues identified numerous AKI risk factors in septic shock, including delayed initiation antibiotic therapy, intra-abdominal sepsis, and blood product transfusion [16]. A detailed metaanalysis about AKI risk factors in sepsis was published by Cartin-Ceba et al. in 2012 [17].

As oposed to sepsis, there are generally less data available about epidemiology and outcome of AKI in tumor patients. A recently published cross-sectional analysis of prospectively collected data revealed an AKI incidence of $12 \%$ in the latter group of patients [18]. In this study AKI risk was significantly correlated with pre-existing diabetes, hyponatremia, intravenous contrast media administration, chemotherapy, and antibiotics. Sepsis in contrast was not identified as risk factor. Another study evaluated predictors of hospital mortality in critically ill cancer patients according to the severity of renal dysfunction. AKI, as defined by the RIFLE criteria [19], occurred in $54.2 \%$ and mortality increased with progression from ' $R$ ' to ' $F$ ' [20]. The results from our study are more or less in line with data from the literature: incidences of AKI were higher in sepsis than in non-septic patients, survival in sepis-associated AKI was lower than in AKI without sepsis or in sepsis without AKI. Frequencies / survival of AKI were higher / lower if a malignant disease was present. Somehow surprising was the observation of comparable mortality rates in non-tumor, nonseptic patients with versus without AKI (15\%). One might argue that due to the absence of sepsis, the overall morbidity of these patients was lower than in septic individuals.

The first new aspect in our study is related to the conincidence of tumor and sepsis and to survival rates of these patients if dialysis therapy became mandatory. In the latter group, mortality increased to almost $70 \%$ (66\%) which once had been reported as the average mortality of AKI patients in the 1970s [21]. A second new aspect is related to mortality in tumor patients with sepsis and AKI, regarding the respective nature of the malignant disease. If a non-solid tumor was diagnosed, mortality was $100 \%$ as compared to patients with nonsolid malignant disease. In those patients mortality was significantly lower (56\%). To our knowledge, this observation has never been reported before and we can only speculate whether non-solid tumors affect the outcome by mechanism related to the disease per se or if the poor outcome results from more aggressive therapeutic interventions. In summary, we conclude that prognosis of tumor patients with AKI and sepsis is very poor. We are well aware of the fact that due to the retrospective and single center-based character of our study, conclusions must be drawn with caution. Thus, further prospective analyses are urgently needed.

\section{Conclusions}

We conclude that prognosis of tumor patients with AKI and sepsis is very poor, with a mortality of approximately $70 \%$ if diaylsis therapy becomes mandatory. Mortality increases even further if patients suffer from a non-solid malignant disease, in this particular group almost 100\% of the patients die.

\section{Key messages}

- Sepsis and AKI significantly worsen prognosis of tumor patients.

- Dialysis treatment in this group is associated with an average mortality of $70 \%$.

- Non-solid tumors are associated with mortality rates of $100 \%$ if patients suffer from sepsis and AKI.

Abbreviations

AKI: Acute kidney injury; ESRD: End-stage renal disease; ICU: Intensice care unit; RIFLE: Risk injury failure loss end-stage renal disease; RRT: Renal. replacement therapy; SOFA: Sequential organ failure assessment.

\section{Competing interests}

The authors declare that they have no competing interests.

Authors' contributions

$\mathrm{MH}$ designed the study and analyzed data. AM collected all data. DE helped in statistical analysis. GAM corrected the manuscript. DP analyzed the data and wrote the manuscript. All authors have nothing to disclose. All authors read and approved the final manuscript.

\section{Author details}

'Department of Nephrology and Rheumatology, University Hospital of Göttingen, Robert-Koch-Straße 40, 37075 Göttingen, Germany. ${ }^{2}$ Department of Medical Statistics, University Hospital of Göttingen, Robert-Koch-Straße 40, 37075 Göttingen, Germany.

Received: 5 August 2013 Accepted: 14 October 2013

Published: 29 October 2013

\section{References}

1. Feldkamp T, Bienholz A, Kribben A: Acute kidney injury. Dtsch Med Wochenschr 2011, 136:194-197.

2. Kribben A, Herget-Rosenthal S, Pietruck F, Philipp T: Acute renal failure-an review. Dtsch Med Wochenschr 2003, 128:1231-1236.

3. Devarajan P: Update on mechanisms of ischemic acute kidney injury. J Am Soc Nephrol 2006, 17:1503-1520.

4. Singbartl K, Kellum JA: AKI in the ICU: definition, epidemiology, risk stratification, and outcomes. Kidney Int 2012, 81:819-825.

5. Murugan R, Kellum JA: Acute kidney injury: what's the prognosis? Nat Rev Nephrol 2011, 7:209-217.

6. Zarjou A, Agarwal A: Sepsis and acute kidney injury. J Am Soc Nephrol 2011, 22:999-1006

7. Ricci Z, Ronco C: Pathogenesis of acute kidney injury during sepsis. Curr Drug Targets 2009, 10:1179-1183.

8. Maccariello E, Valente C, Nogueira L, et al: Outcomes of cancer and noncancer patients with acute kidney injury and need of renal replacement 
therapy admitted to general intensive care units. Nephrol Dial Transplant 2011, 26:537-543.

9. Cruz DN, Ricci Z, Ronco C: Clinical review: RIFLE and AKIN-time for reappraisal. Crit Care 2009, 13:211.

10. Patschan SA, Patschan D, Temme J, et al: Endothelial progenitor cells (EPC) in sepsis with acute renal dysfunction (ARD). Crit Care 2011, 15:R94.

11. Marshall JC: Endotoxin in the pathogenesis of sepsis. Contrib Nephro 2010, 167:1-13

12. Levy MM, Fink MP, Marshall JC, et al: SCCM/ESICM/ACCP/ATS/SIS international sepsis definitions conference. Intensive Care Med 2001, 2003(29):530-538

13. Wan L, Bagshaw SM, Langenberg C, Saotome T, May C, Bellomo R: Pathophysiology of septic acute kidney injury: what do we really know? Crit Care Med 2008, 36:S198-S203.

14. Uchino $S$, Kellum JA, Bellomo $R$, et al: Acute renal failure in critically ill patients: a multinational, multicenter study. JAMA 2005, 294:813-818.

15. Bagshaw SM, Lapinsky S, Dial S, et al: Acute kidney injury in septic shock: clinical outcomes and impact of duration of hypotension prior to initiation of antimicrobial therapy. Intensive Care Med 2009, 35:871-881.

16. Plataki M, Kashani K, Cabello-Garza J, et al: Predictors of acute kidney injury in septic shock patients: an observational cohort study. Clin J Am Soc Nephrol 2011, 6:1744-1751.

17. Cartin-Ceba R, Kashiouris M, Plataki M, Kor DJ, Gajic O, Casey ET: Risk factors for development of acute kidney injury in critically ill patients: a systematic review and meta-analysis of observational studies. Crit Care Res Pract 2012, 2012:691013.

18. Salahudeen AK, Doshi SM, Pawar T, Nowshad G, Lahoti A, Shah P: Incidence rate, clinical correlates, and outcomes of AKI in patients admitted to a comprehensive cancer center. Clin J Am Soc Nephrol 2013, 8:347-354.

19. Bentley W: Towards evidence-based emergency medicine: best BETs from the manchester royal infirmary: BET 3: RIFLE criteria versus acute kidney injury network (AKIN) criteria for prognosis of acute renal failure. Emerg Med J 2011, 28:900-901.

20. Liborio AB, Abreu KL, Silva GBJ, et al: Predicting hospital mortality in critically ill cancer patients according to acute kidney injury severity. Oncology 2011, 80:160-166.

21. Minuth AN, Terrell JBJ, Suki WN: Acute renal failure: a study of the course and prognosis of 104 patients and of the role of furosemide. Am J Med Sci 1976, 271:317-324.

doi:10.1186/1471-2253-13-36

Cite this article as: Heeg et al:: Prognosis of AKI in malignant diseases

with and without sepsis. BMC Anesthesiology 2013 13:36.

Submit your next manuscript to BioMed Central and take full advantage of:

- Convenient online submission

- Thorough peer review

- No space constraints or color figure charges

- Immediate publication on acceptance

- Inclusion in PubMed, CAS, Scopus and Google Scholar

- Research which is freely available for redistribution

Submit your manuscript at www.biomedcentral.com/submit
C Biomed Central 


\section{Danksagung}

Mein Dank gilt Herrn Prof. Dr. med. Daniel Patschan, der mich in die Thematik des akuten Nierenversagens einführte und mir die Grundlage für die Untersuchungen in dieser Arbeit eröffnete. Zu danken habe ich seiner Betreuung, die mir eine große Hilfe war, der Arbeit Struktur und Form zu geben. Den ärztlichen Mitarbeitern der Klinik für Nephrologie und Rheumatologie der Universitätsmedizin Göttingen danke ich für die Unterstützung bei meinen Recherchen im Dickicht der elektronischen Patientendokumentationen. Mein Dank gilt ebenso den Mitarbeitern der Abteilung für Informationstechnologie, die mich vor den Fallstricken der Statistik bewahrten und mir zu statistischen Anwendungen wertvolle Unterstützung gaben. 


\section{Lebenslauf}

Ich wurde am 20.09.1986 in Hannover geboren. Meine Grundschulausbildung erhielt ich in Hannover, Kassel und Genf. Von 1996-1997 besuchte ich die Gymnasialstufe des Engelsburg-Gymnasiums in Kassel und von 1997-2006 das Wilhelmsgymnasium in Kassel, wo ich im Juni 2006 das Abitur ablegte. Im Sommersemester 2007 habe ich zunächst das Studium der Zahnmedizin an der Georg-August-Universität Göttingen aufgenommen und im März 2008 die Naturwissenschaftliche Vorprüfung abgelegt. Ich habe mich dann für einen Wechsel des Studienfachs entschieden und im Oktober 2008 das Studium der Humanmedizin an der Georg-August-Universität Göttingen begonnen. Die Erste Ärztliche Prüfung habe ich im September 2010 erfolgreich bestanden und mit der Zweiten Ärztlichen Prüfung im Oktober 2014 das Medizinstudium beendet. Im praktischen Jahr habe ich in der Chirurgie des Klinikums Lüneburg, in der Inneren Medizin des Katholischen Marienkrankenhauses Hamburg und in der Urologie des Evangelischen Krankenhauses Göttingen-Weende gearbeitet. Seit Juli 2015 bin ich als Assistenzarzt an der Abteilung für Gastroenterologie, Hepatologie und Infektiologie der Universitätsklinik Düsseldorf beschäftigt. 